An Incompressible Two-Dimensional Multiphase Particle-In-Cell Model for Dense Particle Flows

D. M. Snider*

P.J. O'Rourke

M. J. Andrews** 



\section{DISCLAMIER}

Portions of this document may be illegible in electronic image products. Images are produced from the best available original document. 



\section{CONTENTS}

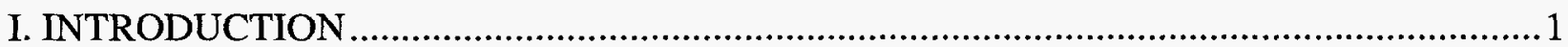

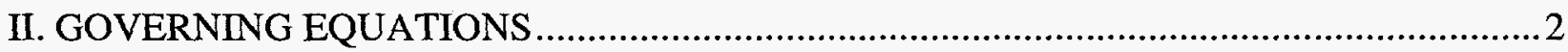

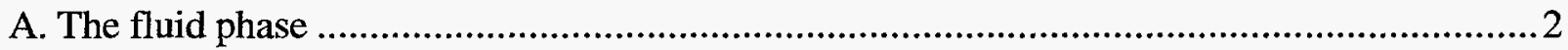

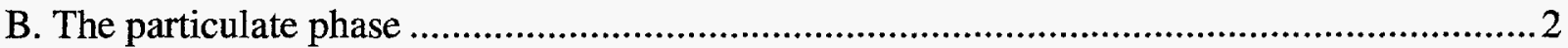

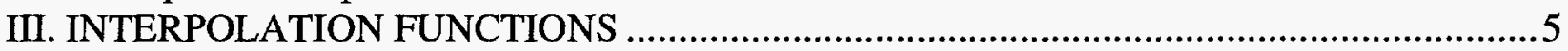

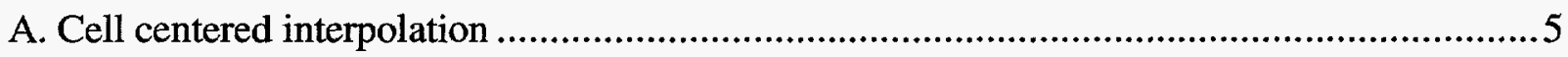

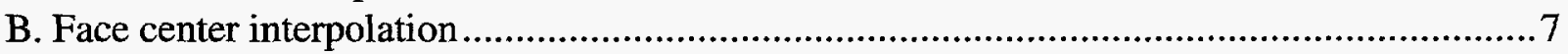

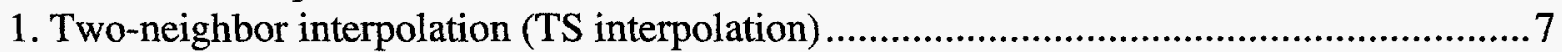

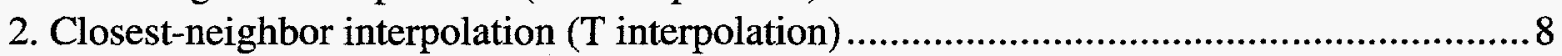

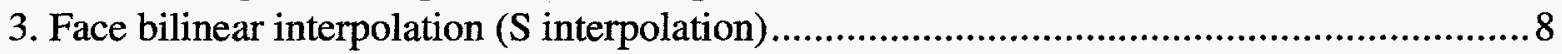

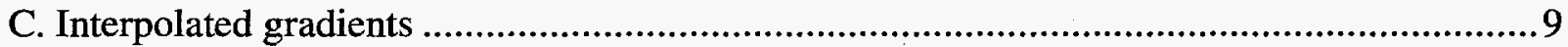

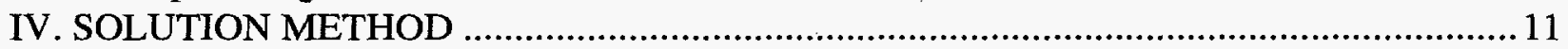

V. PARTICLE EQUATIONS FINITE DIFFERENCE APPROXIMATION ............................11

VI. EULERIAN DIFFERENCE APPROXIMATIONS ….................................................. 12

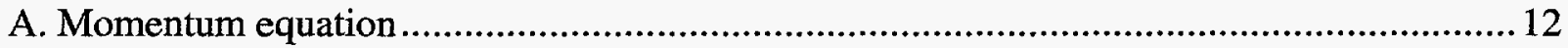

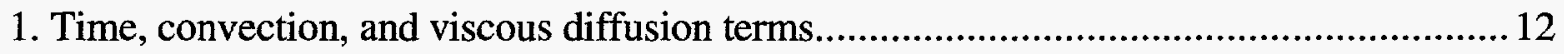

2. Interphase momentum transfer .................................................................................... 13

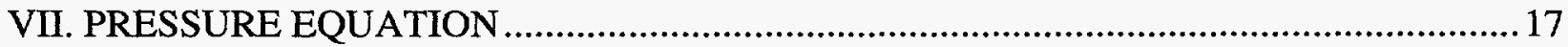

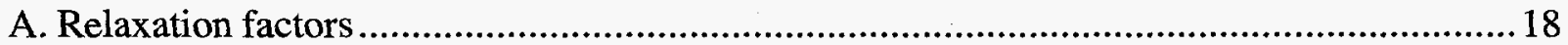

VIII. PARTICLE VOLUME FRACTION ON THE EULERIAN GRID …...........................18

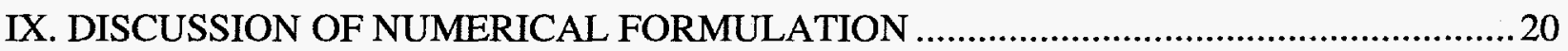

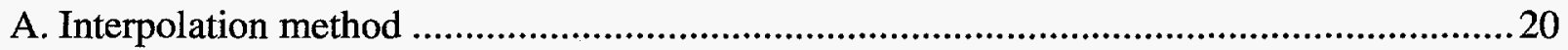

1. Interpolation of the continuum properties to particle positions .....................................20

2. Interpolation of the particle properties to the Eulerian grid..........................................22

B. Calculated layering of particles in a quasi-steady fluidized bed........................................22

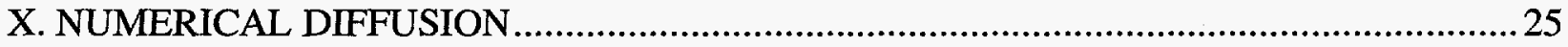

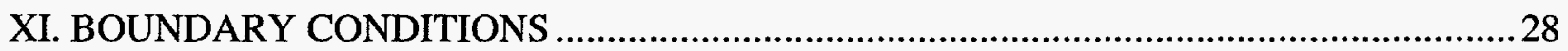

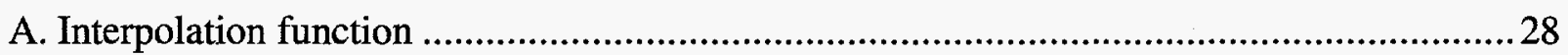

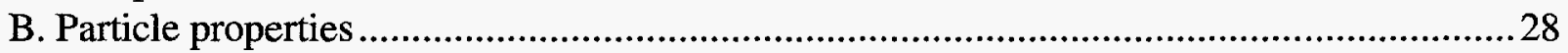

C. Fluid properties......................................................................................................... 29

XII. APPROXIMATION OF THE IMPLICIT PARTICLE VOLUME FRACTION .................29

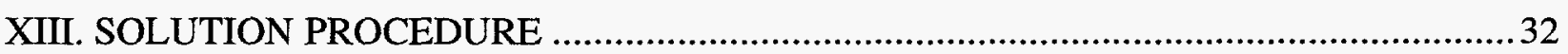

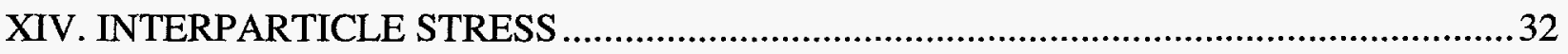

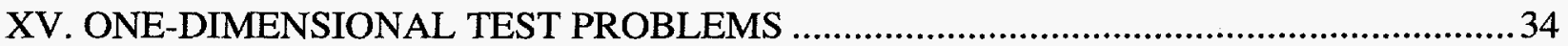

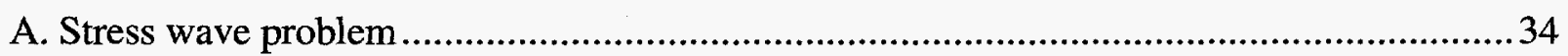

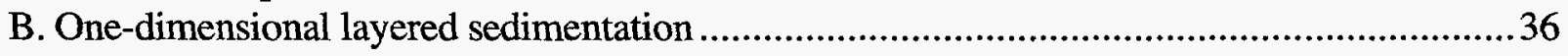

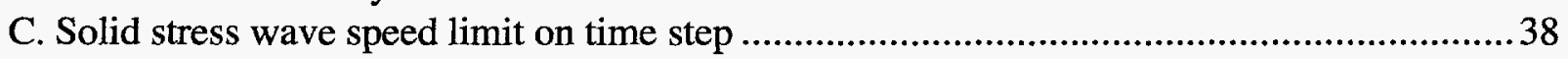

XVI. TWO-DIMENSIONAL HEAVY PARTICLE MIXTURE ABOVE A LIGHT FLUID ......38

A. Rayleigh-Taylor single wave instability............................................................................39

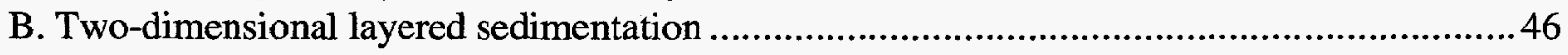

C. Two-dimensional unstable buoyancy mixing layer..............................................................4

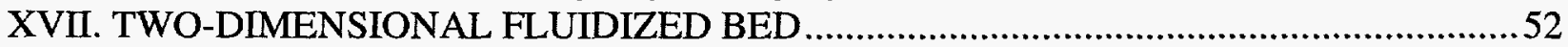

A. Fluidized bed calculation for experiment by Lin, et al .....................................................5 
1. Grid independence

2. An observation on two-dimensional MP-PIC calculations in cylindrical coordinates

B. Fluidized bed calculation for experiment by Ding and Gidaspow

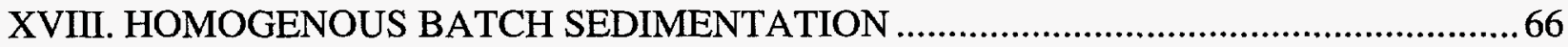

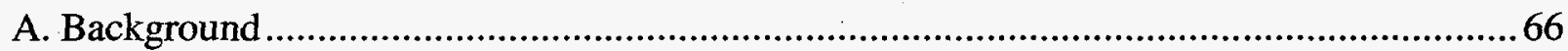

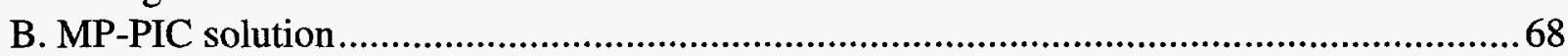

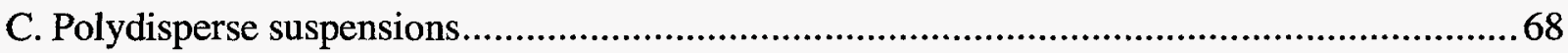

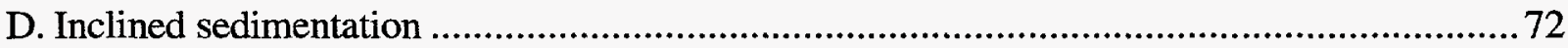

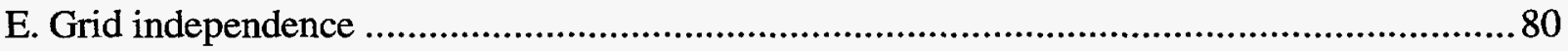

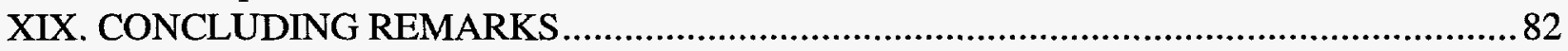

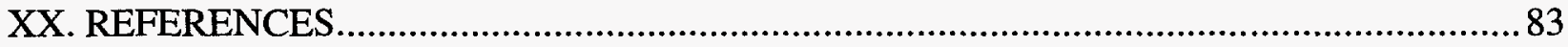

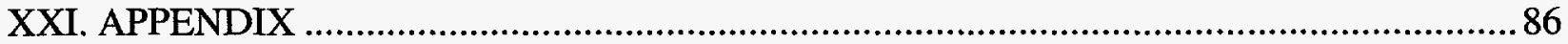

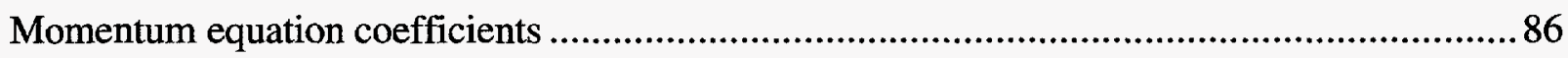

Pressure equation coefficients ............................................................................ 91

Particle volume fraction equation coefficients ....................................................... 94 


\section{FIGURES}

Figure 1. The $\mathrm{S}$ and $\mathrm{T}$ interpolation functions. .5

Figure 2. Interpolation from Eulerian grid to a particle position. ............................................6

Figure 3. Velocity interpolation from Eulerian grid to a particle position......................................8

Figure 4. The $\mathrm{x}$-velocities used in interpolating the grid velocity to particle position $\mathbf{x}_{p}$...............

Figure 5. Momentum finite difference volume for $x$-vector component. ..................................... 12

Figure 6. Continuity equation finite difference volume. .............................................................. 17

Figure 7. The 9 point pressure stencil for the pressure equation................................................. 18

Figure 8. Rayleigh-Taylor calculation. Left: uses $T S$ interpolation function from Eulerian grid to particle position. Right: uses bilinear interpolation function from Eulerian grid to

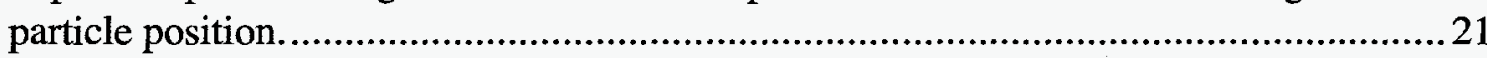

Figure 9. Unique particle position in a fluidized bed from $\theta_{g}$ interpolation to particle location. ...24 Figure 10. Particle flow between flat plates. Gas velocity at particle position from $S^{S}$.................26

Figure 11. Fluid velocity profile for particle flow between flat plates. Gas velocity at particle position from $S^{S}$.

Figure 12. Particle flow between flat plates. Gas velocity at particle position from $S^{T}$...............22

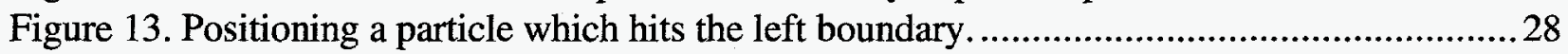

Figure 14. The implicit update of particle volume fraction......................................................30

Figure 15. Diagram of calculation scheme with implicit particle volume fraction. ........................33

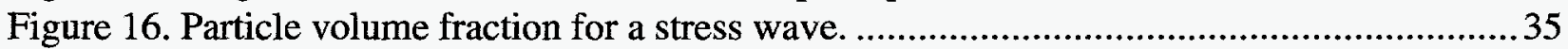

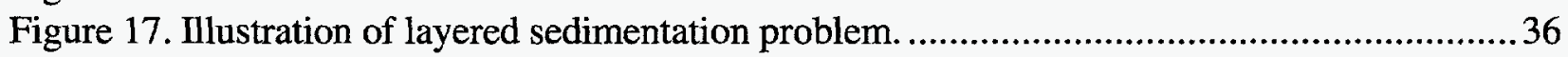

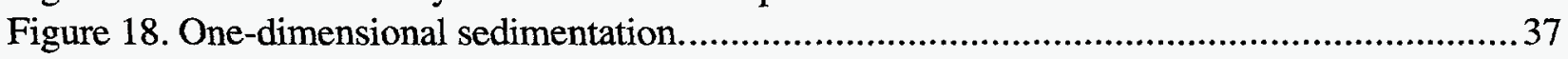

Figure 19. Stress wave courant number for the 1-D sedimentation problem. …….......................39

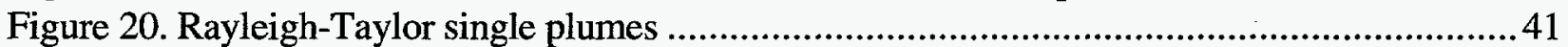

Figure 21. Comparison of calculated Rayleigh-Taylor plume growth rate with linear stability growth rate and measured saturated growth rate $\left(\mathrm{C}_{\infty}=0.7\right)$.

Figure 22. Comparison of calculated Rayleigh-Taylor plume growth rate with linear stability growth rate and measured saturated growth rate $\left(\mathrm{C}_{\infty}=0.7\right)$. Calculated curves are adjusted to a zero virtual origin. ............................................................................... 42

Figure 23. Rayleigh-Taylor single wave perturbation with grid resolution of $20 \times 100 \ldots \ldots \ldots \ldots \ldots \ldots . . . .44$

Figure 24. Rayleigh-Taylor single wave perturbation with grid resolution of $20 \times 100$ and 7,823 and 19,560 particles

Figure 25. Two-dimensional layered sedimentation, comparable to the one-dimensional layered sedimentation problem.

Figure 26. Unstable buoyancy mixing layer. $\rho_{\mathrm{p}}=6 \mathrm{~kg} / \mathrm{m}^{3}, \rho_{\mathrm{g}}=1 \mathrm{~kg} / \mathrm{m}^{3}$, initial $\theta_{\mathrm{p}}=0.2$, $\mathrm{g}=147 \mathrm{~m} / \mathrm{s}^{2}$ 50

Figure 27. Unstable buoyancy mixing layer growth rate. .51

Figure 28. Fluidized bed. Dimensions and gas flow rate are from experiments by

Lin, et al. (1985)

Figure 29. MP-PIC calculated particle volume fraction field at time intervals for the experiment by Lin, et al. (1985). Inlet flow $=64 \mathrm{~cm} / \mathrm{s}$ and $P_{s}=75 \mathrm{~Pa}$. Radial nodes $=6$. Axial nodes $=15$. .54

Figure 30. Lin, et al. (1985) illustrated recalculation behavior for high fluidizing velocity.............55 Figure 31. MP-PIC calculated particle velocity field for the experiment by Lin, et al. (1985). 
Inlet flow $=64 \mathrm{~cm} / \mathrm{s}$ and $P_{s}=75 \mathrm{~Pa}$. Radial nodes $=6$. Axial nodes $=15$

Figure 32. MP-PIC calculated instantaneous and average particle volume fraction compared with that measured by Lin, et al. (1985). Inlet flow $=64 \mathrm{~cm} / \mathrm{s}$ and $P_{\mathrm{s}}=75 \mathrm{~Pa}$. Radial nodes $=6$. Axial nodes $=15$.

Figure 33. MP-PIC calculated particle volume fraction field at time intervals for the experiment by Lin, et al. (1985). Inlet flow $=64 \mathrm{~cm} / \mathrm{s}$ and $P_{\mathrm{s}}=75 \mathrm{~Pa}$. Radial nodes $=12$. Axial nodes $=30$.

Figure 34. MP-PIC calculated particle velocity field for the experiment by Lin, et al. (1985).

Inlet flow $=64 \mathrm{~cm} / \mathrm{s}$ and $P_{s}=75 \mathrm{~Pa}$. Radial nodes $=12$. Axial nodes $=30$.

Figure 35. MP-PIC calculated average particle volume fraction compared with that measured

by Lin, et al. (1985). Inlet flow $=64 \mathrm{~cm} / \mathrm{s}$ and $\mathrm{P}_{\mathrm{s}}=75 \mathrm{~Pa}$

Figure 36. Particle distribution in the IIT fluidized bed. Inlet flow $=1 \mathrm{~m} / \mathrm{s}, \theta_{i}=0.4$.

Figure 37. Particle volume fraction field in the IIT fluidized bed. Inlet flow $=1 \mathrm{~m} / \mathrm{s}, \theta_{i}=0.4$....64

Figure 38. IIT fluidized bed average particle velocities. Inlet flow $=100 \mathrm{~cm} / \mathrm{s}, \theta_{i}=0.3$. .65

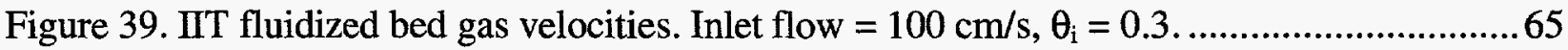

Figure 40 . Illustration of a vertical bimodal suspension. ...................................................66

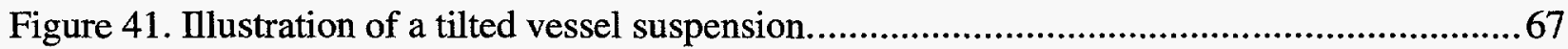

Figure 42. Particle distribution for batch sedimentation (Davis, et al., 1982). ........................ 70

Figure 43. Sedimentation levels from bimodal suspension of particles (Davis, et al., 1982)........ 71

Figure 44. The particle distributions for batch sedimentation at $400 \mathrm{~s}$ with the container inclined $0^{\circ}, 20^{\circ}$, and $35^{\circ}$

Figure 45. Batch sedimentation. Container tilted $35^{\circ}$.

Figure 46. Batch sedimentation. Container tilted $35^{\circ}$ and $t=300 \mathrm{~s}$. MP-PIC calculation with

$30 \mathrm{x}$-nodes, $88 \mathrm{y}$-nodes and 15,000 particles.

Figure 47. MP-PIC calculated and measured interface for suspension of particles (Acrivos \&

Herbolzheimer 1979). 77

Figure 48. Fluid velocity for container tilted $35^{\circ}$.

Figure 49. Particle velocity for container tilted $35^{\circ}$. Particle velocity is from mapping particles to the Eulerian grid.

Figure 50. Observation of the flow for a volume fraction of 0.01 and fluid viscosity of $18.8 \mathrm{cP}$.

(a) angle of inclination large; (b) angle is decreased and waves appear along interface in the upper region of channel.

Figure 51. Batch sedimentation. Fine grid spacing. Particle distribution at $300 \mathrm{~s}$ for container tilted $35^{\circ}$. 


\section{TABLES}

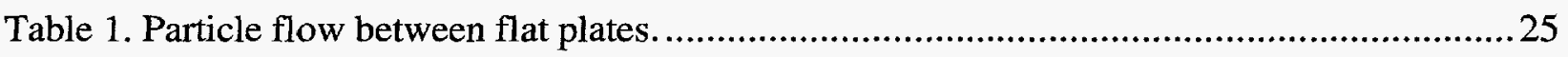

Table 2. Stress wave problem with a single $x$-channel. ..................................................... 34

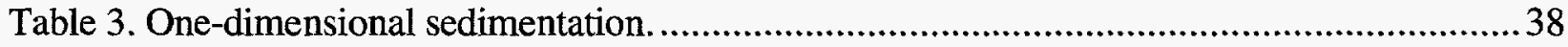

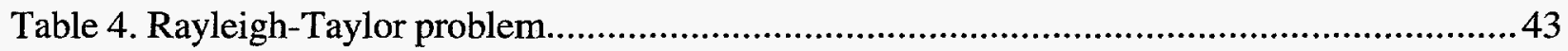

Table 5. Rayleigh-Taylor problem with nodalization of $20 \times 100$ and 7,823 and 19,560

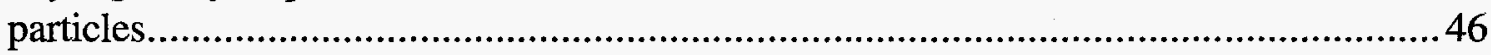

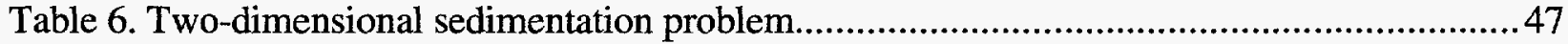

Table 7. Layered sedimentation comparable to experiments by Youngs (1989). ......................48

Table 8. Two-dimensional fluidized bed problem with conditions for the experiment by

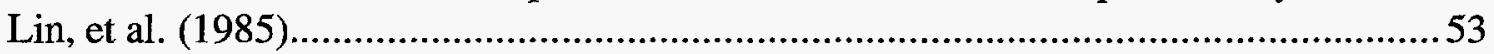

Table 9. IIT two-dimensional fluidized bed problem. ...................................................62

Table 10. Batch bimodal sedimentation (Davis, et al., 1982)............................................. 69

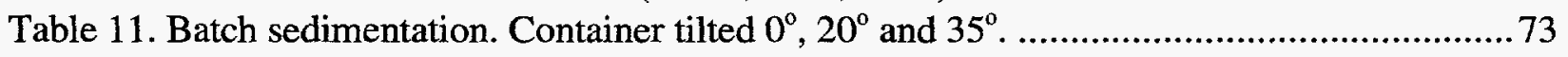





\title{
AN INCOMPRESSIBLE TWO-DIMENSIONAL MULTIPHASE PARTICLE-IN-CELL MODEL FOR DENSE PARTICLE FLOWS
}

\author{
by \\ D. M. Snider, P. J. O'Rourke, and M. J. Andrews
}

\begin{abstract}
A two-dimensional, incompressible, multiphase particle-in-cell (MP-PIC) method is presented for dense particle flows. The numerical technique solves the governing equations of the fluid phase using a continuum model and those of the particle phase using a Lagrangian model. Difficulties associated with calculating interparticle interactions for dense particle flows with volume fractions above 5\% have been eliminated by mapping particle properties to an Eulerian grid and then mapping back computed stress tensors to particle positions. This approach utilizes the best of Eulerian/Eulerian continuum models and Eulerian/Lagrangian discrete models. The solution scheme allows for distributions of types, sizes, and density of particles, with no numerical diffusion from the Lagrangian particle calculations. The computational method is implicit with respect to pressure, velocity, and volume fraction in the continuum solution thus avoiding courant limits on computational time advancement. MP-PIC simulations are compared with onedimensional problems that have analytical solutions and with two-dimensional problems for which there are experimental data.
\end{abstract}

\section{INTRODUCTION}

A two-dimensional, incompressible, multiphase particle-in-cell (MP-PIC) method has been implemented for dense particle flows. The numerical technique utilizes the best of Eulerian/Eulerian continuum models and Eulerian/Lagrangian discrete models. Computational simulations of batch sedimentation, layered sedimentation, and fluidized beds are given to illustrate the technique.

Mathematical models of separated particulate multiphase flow have used either a continuum approach for all phases (Gidaspow 1986, Batchelor 1988) or a continuum for the fluid and a Lagrangian model for particles (Amsden, et al., 1989). The continuum/continuum model readily allows modeling of particle-particle stresses in dense particle flows using spatial gradients of particle volume fractions (Batchelor 1988, Gidaspow 1994). However, modeling a distribution of types and sizes of particles complicates the continuum formulation because separate continuity and momentum equations must be solved for each size and type (Risk 1993, Gidaspow 1994). Using a continuum model for the fluid phase and a Lagrangian model for particle phase allows economical solution for flows with a wide range of particle types, sizes, shapes and velocities (O'Rourke 1981, Gidaspow 1994). However, the collision frequency is high for volume fractions above $5 \%$ and cannot be realistically resolved by current Lagrangian collision calculations (O'Rourke 1981).

The two-dimensional MP-PIC method presented here provides a numerical scheme whereby the particle phase is treated as both a continuum and as discrete particles, gaining the best of both 
methods. Particle properties are mapped to and from an Eulerian grid. While on the grid, continuum derivatives that treat the particle phase as a fluid are evaluated and then mapped back to the individual particles. The result of this procedure is a computational technique for multiphase flow that can handle particle loadings ranging from dilute to dense with a distribution of particle materials and sizes.

The two-dimensional, MP-PIC method extends the one-dimensional work by Andrews and O'Rourke (1996). The two-dimensional MP-PIC method adds the normal complexity of extending a numerical scheme from one to two dimensions. The linear mapping to and from the Eulerian grid is extended to a bilinear interpolation which adds an order of magnitude more terms in the resulting algebraic equations. Further, the numerical solution here is an incompressible continuum solution which requires formulation and solution of a pressure equation. The two-dimensional scheme allows for solution of a broader range of flow problems where currents form from obstructions or physical instabilities. To illustrate the two-dimensional MP-PIC method, calculations for fluidized beds, Rayleigh-Taylor heavy particle mixtures initially above a light continuum, and homogenous sedimentation are presented and compared with experimental data.

\section{GOVERNING EQUATIONS}

The governing equations for the continuum and particles are given. The fluid phase is incompressible and inviscid, and fluid and particle phases are isothermal. The fluid or Eulerian variables are denoted by subscript " $g$ ", and the particle or Lagrangian variables are denoted by subscript " $p$ ".

\section{A. The fluid phase}

The continuity equation for the fluid with no interphase mass transfer is

$$
\frac{\partial \theta_{g}}{\partial t}+\nabla_{\mathbf{x}} \cdot\left(\theta_{g} \mathbf{u}_{g}\right)=0,
$$

where $\mathbf{u}_{g}$ is the fluid velocity and $\theta_{g}$ is the fluid volume fraction.

The momentum equation for the fluid is

$$
\frac{\partial\left(\theta_{g} \mathbf{u}_{g}\right)}{\partial t}+\nabla_{\mathbf{x}} \cdot\left(\theta_{g} \mathbf{u}_{g} \mathbf{u}_{g}\right)=-\frac{1}{\rho_{g}} \nabla_{\mathbf{x}} p+\nabla\left(\theta_{g} v_{g} \nabla \mathbf{u}_{g}\right)-\frac{1}{\rho_{g}} \mathbf{F}+\theta_{g} \mathbf{g}
$$

where $\rho_{g}$ is fluid density, $v_{g}$ is fluid kinematic viscosity, $p$ is fluid pressure and $\mathbf{g}$ is the gravitational acceleration. $\mathbf{F}$ is the rate of momentum exchange per volume between the fluid and particles phases and will be defined later.

\section{B. The particulate phase}

Following Andrews and O'Rourke (1996), the dynamics of the particle phase is described using the particle probability distribution function $f\left(\mathbf{x}, \mathbf{u}_{p}, \rho_{p}, V_{p}, t\right)$, where $\mathbf{x}$ is the particle position, $\mathbf{u}_{p}$ is the particle velocity, $\rho_{p}$ is the particle density and $V_{p}$ is the particle volume. For the present it is assumed that the mass of each particle is constant in time (no mass transfer between particles or 
to the fluid), but particles may have a range of sizes and densities. The probability function integrated over velocity and mass gives the probable number of particles per unit volume at $\mathbf{x}$ and $t$ in the interval $\left(\mathbf{u}_{p}, \mathbf{u}_{p}+\mathrm{d} \mathbf{u}_{p}\right),\left(\rho_{p}, \rho_{p}+d \rho_{p}\right)$ and $\left(V_{p}, V_{p}+d V_{p}\right)$. The particle volume fraction is defined from the particle distribution function as

$$
\theta_{p}=\iiint f V_{p} d V_{p} d \rho_{p} d \mathbf{u}_{p}
$$

The sum of volume fractions of fluid and particle phases must equal unity,

$$
\theta_{p}+\theta_{g}=1
$$

The time evolution of $f$ is obtained by solving a Liouville equation for the particle distribution function (Williams 1985)

$$
\frac{\partial f}{\partial t}+\nabla_{\mathbf{x}} \cdot\left(f \mathbf{u}_{p}\right)+\nabla_{\mathbf{u}_{p}} \cdot(f \mathbf{A})=0
$$

where the particle acceleration, $\mathbf{A}=d \mathbf{u}_{\mathrm{p}} / d t$, is given by

$$
\mathbf{A}=D\left(\mathbf{u}_{g}-\mathbf{u}_{p}\right)-\frac{1}{\rho_{p}} \nabla_{\mathbf{x}} p+\mathbf{g}-\frac{1}{\theta_{p} \rho_{p}} \nabla_{\mathbf{x}} \tau .
$$

The terms in (6) represent acceleration due to aerodynamic drag, pressure gradient, gravity and gradients in the interparticle stress, $\tau$.

The drag model used here is the same as used by Andrews and O'Rourke (1996),

$$
D_{p}=C_{d} \frac{3}{8} \frac{\rho_{g}}{\rho_{p}} \frac{\left|\mathbf{u}_{g}-\mathbf{u}_{p}\right|}{r},
$$

where

$$
C_{d}=\frac{24}{\operatorname{Re}}\left(\theta_{g}^{-2.65}+\frac{1}{6} \operatorname{Re}^{2 / 3} \theta_{g}^{-1.78}\right)
$$

The Reynolds number is defined as

$$
\operatorname{Re}=\frac{2 \rho_{g}\left|\mathbf{u}_{g}-\mathbf{u}_{p}\right| r}{\mu_{g}},
$$

where $\mu_{g}$ is the gas viscosity and the particle radius is

$$
r=\left(\frac{3 V_{p}}{4 \pi}\right)^{1 / 3}
$$


Collisions between particles are modeled by an isotropic interparticle stress from Harris and Crighton (1994),

$$
\tau=\frac{P_{s} \theta_{p}^{\beta}}{\theta_{c p}-\theta_{p}}
$$

where the constant $P_{s}$ has units of pressure and $\theta_{c p}$ is the particle volume fraction at close packing. The power $\beta$ on the volume fraction forces the interparticle stress to be significant only as particles become closely packed. For $\beta=1$, an interparticle stress gradient exists even at zero volume fraction. For $\beta>1$, the interparticle stress gradient is zero at zero volume fraction. Auzeris et al. (1988) recommend $2 \leq \beta \leq 5$.

The interphase momentum transfer function per volume is obtained from

$$
\mathbf{F}=\iiint f V_{p} \rho_{p}\left[D_{p}\left(\mathbf{u}_{g}-\mathbf{u}_{p}\right)-\frac{1}{\rho_{p}} \nabla p\right] d V_{p} d \rho_{p} d \mathbf{u}_{p} .
$$

The Eulerian governing equations for the particle phase may be obtained by taking the moments of equation (5). By multiplying equation (5) by $\rho_{p} V_{p}$ and $\rho_{p} V_{p} \mathbf{u}_{\mathrm{p}}$ and integrating over particle density, volume, and velocity coordinates (Travis et al., 1976), the particle conservation equations are obtained,

$$
\frac{\partial\left(\overline{\theta_{p} \rho_{p}}\right)}{\partial t}+\nabla_{\mathbf{x}} \cdot\left(\overline{\theta_{p} \rho_{p}} \overline{\mathbf{u}}_{p}\right)=0
$$

and

$$
\begin{aligned}
& \frac{\partial\left(\overline{\theta_{p} \rho_{p}} \overline{\mathbf{u}}_{p}\right)}{\partial t}+\nabla_{\mathbf{x}} \cdot\left(\overline{\theta_{p} \rho_{p}} \overline{\mathbf{u}}_{p} \overline{\mathbf{u}}_{p}\right)=-\theta_{p} \nabla_{\mathbf{x}} p-\nabla_{\mathbf{x}} \tau+\overline{\theta_{p} \rho_{p}} \mathbf{g} \\
& \quad+\iiint f V_{p} \rho_{p} D_{p}\left(\mathbf{u}_{g}-\mathbf{u}_{p}\right) d V_{p} d \rho_{p} d \mathbf{u}_{p} \\
& -\nabla_{\mathbf{x}} \cdot\left[\iiint f V_{p} \rho_{p}\left(\mathbf{u}_{p}-\overline{\mathbf{u}}_{p}\right)\left(\mathbf{u}_{p}-\overline{\mathbf{u}}_{p}\right) d V_{p} d \rho_{p} d \mathbf{u}_{p}\right]
\end{aligned}
$$

where the mean particle velocity $\overline{\mathbf{u}}_{p}$ is given by

$$
\overline{\mathbf{u}}_{p}=\frac{1}{\overline{\theta_{p} \rho_{p}}} \iiint f V_{p} \rho_{p} \mathbf{u}_{p} d V_{p} d \rho_{p} d \mathbf{u}_{p},
$$

and the average particle density is given by

$$
\overline{\theta_{p} \rho_{p}}=\iiint f V_{p} \rho_{p} d V_{p} d \rho_{p} d \mathbf{u}_{p}
$$




\section{INTERPOLATION FUNCTIONS}

Particle properties are mapped to and from the Eulerian grid as part of the numerical solution. Because a staggered grid is employed, scalar properties, defined at cell centers, are mapped with one set of interpolation functions, and face center velocities are mapped with another set. This section describes the interpolation functions for cell center properties, face center properties and gradients.

\section{A. Cell centered interpolation}

Particle properties are interpolated to and from an Eulerian cell center node, using a bilinear interpolation function, $\mathrm{S}$. The bilinear interpolation function is formed from the product of linear interpolation functions in the $\mathrm{x}$ and $\mathrm{y}$ directions,

$$
S_{i, j}=S_{x_{i}} S_{y_{j}}
$$

Figure 1 shows the $S_{x_{i}}$ function. The x-interpolation function, $S_{x_{i}}$, which is independent of the $\mathrm{y}$ coordinate, is unity at node $(i, j)$ and decreases linearly to zero at neighbor nodes $(i-1, j)$ and $(i+1, j)$. Similarly, $S_{y_{j}}$ which is independent of the x-coordinate, is unity at $(i, j)$ and goes to zero at its adjacent nodes. Thus the bilinear interpolation function, $S_{i, j}$, which is dependent on both $\mathrm{x}$ and $y$-coordinates, is unity at the cell center $(i, j)$ and decreases to zero at the surrounding eight nodes.
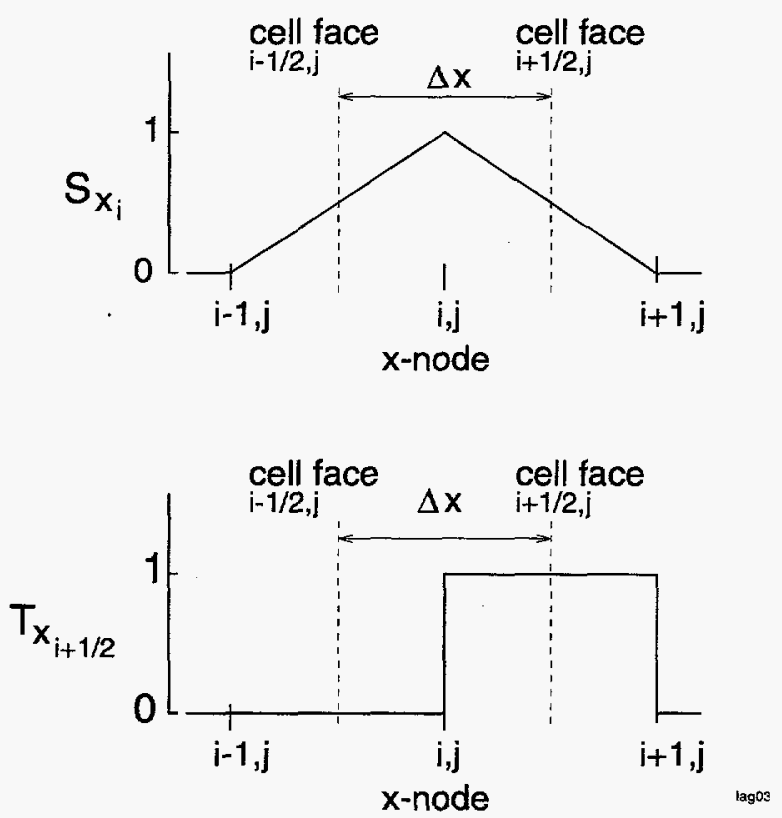

Figure 1. The $\mathrm{S}$ and $\mathrm{T}$ interpolation functions.

Figure 2 shows a particle at $\mathbf{x}_{p}$ on the Eulerian grid bounded by four cell center nodes. The $S$ interpolation functions for interpolating to particle location $\left(x_{p}, y_{p}\right)$ are 


$$
\begin{gathered}
S_{i, j}=S_{x_{i}} S_{y_{j}} \\
S_{i+1, j}=\left(1-S_{x_{i}}\right) S_{y_{j}} \\
S_{i+1, j+1}=\left(1-S_{x_{i}}\right)\left(1-S_{y_{j}}\right) \\
S_{i, j+1}=S_{x_{i}}\left(1-S_{y_{j}}\right) .
\end{gathered}
$$

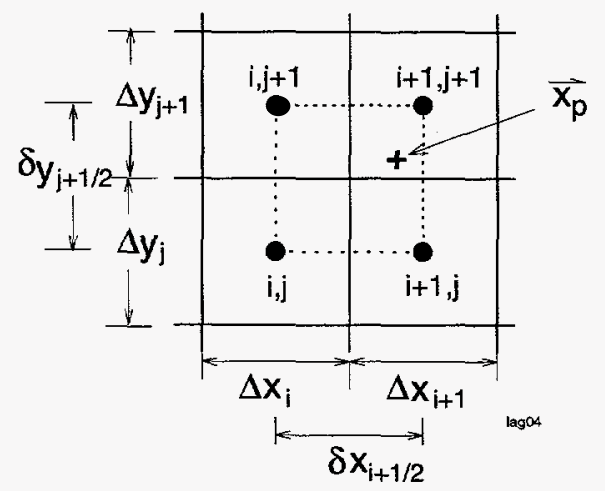

Figure 2. Interpolation from Eulerian grid to a particle position.

The $S_{x}$ and $S_{y}$ interpolation functions at $\mathbf{x}_{\mathrm{p}}$ for a rectangular grid are

$$
\begin{gathered}
S_{x_{i}}=\frac{\left(x_{i+1}-x_{p}\right)}{\delta x_{i+1 / 2}} \\
S_{y_{j}}=\frac{\left(y_{j+1}-y_{p}\right)}{\delta y_{j+1 / 2}},
\end{gathered}
$$

and $\delta x_{i+1 / 2}=\left(x_{i+1}-x_{i}\right)$ and $\delta y_{j+1 / 2}=\left(y_{j+1}-y_{j}\right)$. A cell centered continuum property, $\psi$, interpolated to the particle position, $\mathbf{x}_{p}$, is given by

$$
\psi_{\mathrm{p}}=\mathrm{S}\left(\mathbf{x}_{\mathrm{p}}\right)_{\mathrm{i}, \mathrm{j}} \psi_{\mathrm{i}, \mathrm{j}}+\mathrm{S}\left(\mathbf{x}_{\mathrm{p}}\right)_{\mathrm{i}+1, \mathrm{j}} \psi_{\mathrm{i}+1, \mathrm{j}}+\mathrm{S}\left(\mathbf{x}_{\mathrm{p}}\right)_{\mathrm{i}+1, \mathrm{j}+1} \psi_{\mathrm{i}+1, \mathrm{j}+1}+\mathrm{S}\left(\mathbf{x}_{\mathrm{p}}\right)_{\mathrm{i}, \mathrm{j}+1} \psi_{\mathrm{i}, \mathrm{j}+1}
$$

In this study, the particle volume fraction, $\theta_{p}$, is mapped to the Eulerian grid by 


$$
\theta_{p_{i, j}}=\frac{1}{V_{i, j}} \sum_{p} N_{p} V_{p} S\left(\mathbf{x}_{p}\right)_{i, j}
$$

where $N_{p}$ is the number of particles in a parcel. The Eulerian cell volume is $V_{i, j}$.

\section{B. Face center interpolation}

Three methods of interpolating particle properties to and from volume faces were considered. The first interpolation method is a linear interpolation using the two face-centers bounding a particle, the second method uses the closest face, and the third method is a bilinear interpolation using the four face centers enclosing the particle. It was found that the bilinear interpolation method, which is similar to that for cell center interpolation, was required for interpolating to particle positions and a closest-face interpolation to the grid was sufficient to obtain an acceptably smooth distribution of particles with the current model. The characteristics and advantages of the three methods are discussed later.

\section{Two-neighbor interpolation (TS interpolation)}

The two-neighbor interpolation uses the face center u-velocities above and below particles, and the face center $v$-velocities to the left and right of particles. Figure 3 shows $\mathbf{u}$ and v-velocities used in interpolating to a particle located at position " + ". The interpolation is a linear interpolation function in one direction multiplied by a top-hat interpolation function in the other direction and is designated as a $T S$ interpolation function. The $T S$ interpolation functions for u-velocities are

$$
\begin{gathered}
S_{i+1 / 2, j}^{T S}=T_{i+1 / 2} S_{y_{j}} \\
S_{i+1 / 2, j+1}^{T S}=T_{i+1 / 2}\left(1-S_{y_{j}}\right)
\end{gathered}
$$

and the TS interpolation functions for v-velocities are

$$
\begin{gathered}
S_{i, j+1 / 2}^{T S}=S_{x_{i}} T_{j+1 / 2} \\
S_{i+1, j+1 / 2}^{T S}=\left(1-S_{x_{i}}\right) T_{j+1 / 2} .
\end{gathered}
$$

The fluid velocities interpolated to particle position $\mathbf{x}_{p}$ are

$$
\begin{aligned}
& u_{g, p}=S\left(\mathbf{x}_{p}\right)_{i+1 / 2, j}^{T S} u_{g_{i+1 / 2, j}}+S\left(\mathbf{x}_{p}\right)_{i+1 / 2, j+1}^{T S} u_{g_{i+1 / 2, j+1}} \\
& v_{g, p}=S\left(\mathbf{x}_{p}\right)_{i, j+1 / 2}^{T S} v_{g_{i, j+1 / 2}}+S\left(\mathbf{x}_{p}\right)_{i+1, j+1 / 2}^{T S} v_{g_{i+1, j+1 / 2}} .
\end{aligned}
$$


The top-hat $T_{i+1 / 2}$ function, shown in Figure 1, is unity between computational cell centers and zero everywhere else.

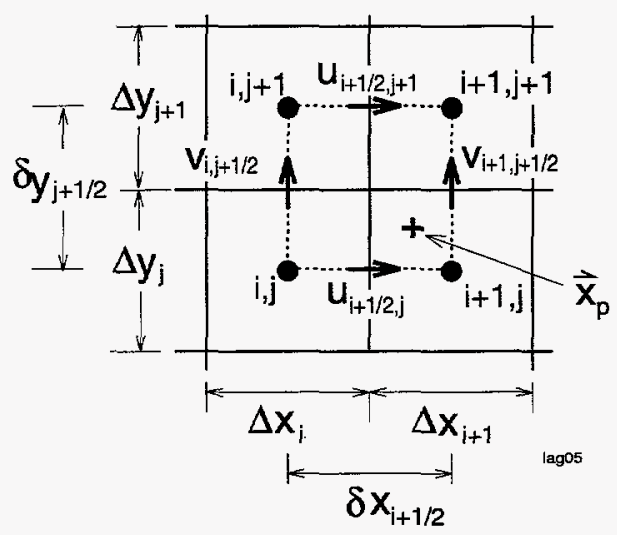

Figure 3. Velocity interpolation from Eulerian grid to a particle position.

\section{Closest-neighbor interpolation ( $T$ interpolation)}

The closest-neighbor interpolation uses the velocity from the nearest face. Figure 3 shows face $u$ and $v$-velocities used in interpolating to a particle located at position " $+"$. This interpolation is formed from two $T$ interpolation functions. For u-velocities, $S_{i+1 / 2, j}^{T}=T_{i+1 / 2} T_{j}$ and for vvelocities, $S_{i, j+1 / 2}^{T}=T_{i} T_{j+1 / 2}$. The $T$, top-hat function, is unity between computational cell centers and zero everywhere else. Velocities are interpolated using equations (24a) and (24b), replacing the TS interpolation functions with $\mathrm{T}$ interpolation functions.

\section{Face bilinear interpolation ( $S$ interpolation)}

Figure 4 shows that the interpolated fluid velocity at particle position $\mathbf{x}_{p}$ may be better represented using four encompassing face velocities instead of two. The face interpolation functions have grid support different from cell center interpolation, and the grid support for the $\mathrm{x}$ face is different from that for the $y$-face. For a $u$-velocity the interpolation function is

$$
S_{i+1 / 2, j}^{S}=S_{x_{i+1 / 2}} S_{y_{j}}
$$

and for the v-velocity, the $S$ interpolation function is

$$
S_{i, j+1 / 2}^{S}=S_{x_{i}} S_{y_{j+1 / 2}}
$$

where $S_{x_{i+1 / 2}}$ and $S_{y_{j+1 / 2}}$ are linear interpolation functions between face-centers and $S_{x_{i}}$ and $S_{y_{j}}$ are previously defined linear cell-center interpolation functions. The fluid velocities interpolated to particle position $\mathbf{x}_{p}$ are

$$
u_{g, p}=S_{i-1 / 2, j}^{S}\left(\mathbf{x}_{p}\right) u_{g_{i-1 / 2, j}}+S_{i+1 / 2, j}^{S}\left(\mathbf{x}_{p}\right) u_{g_{i+1 / 2, j}}
$$




$$
\begin{aligned}
& +S_{i+1 / 2, j+1}^{S}\left(\mathbf{x}_{p}\right) u_{g_{i+1 / 2, j+1}}+S_{i-1 / 2, j+1}^{S}\left(\mathbf{x}_{p}\right) u_{g_{i-1 / 2, j+1}} \\
v_{g, p}= & S_{i, j-1 / 2}^{S}\left(\mathbf{x}_{p}\right) v_{g_{i, j-1 / 2}}+S_{i+1, j-1 / 2}^{S}\left(\mathbf{x}_{p}\right) v_{g i+1, j-1 / 2} \\
& +S_{i+1, j+1 / 2}^{S}\left(\mathbf{x}_{p}\right) v_{g i+1, j+1 / 2}+S_{i, j+1 / 2}^{S}\left(\mathbf{x}_{p}\right) v_{g_{i, j+1 / 2}}
\end{aligned}
$$

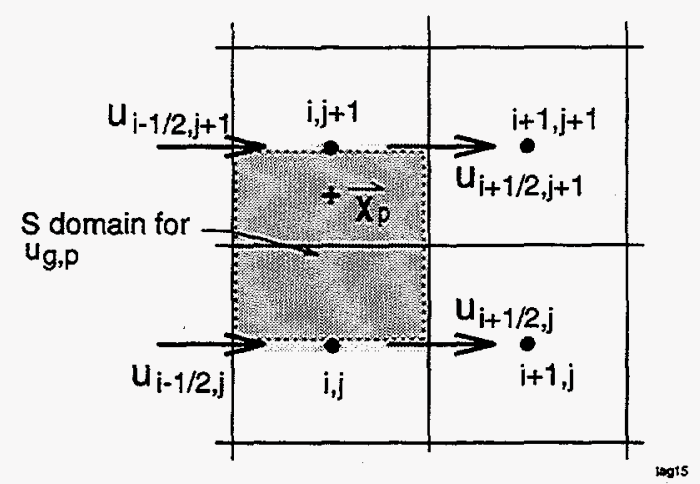

Figure 4. The $\mathrm{x}$-velocities used in interpolating the grid velocity to particle position $\mathbf{x}_{p}$.

\section{Interpolated gradients}

The gradient of the continuum property $\psi$ at particle location $x_{p}$ surrounded by the four nodes shown in Figure 2 is

$$
\nabla \Psi_{p}=\sum_{k=1}^{4} \Psi_{k} \nabla S\left(\mathbf{x}_{p}\right)_{k}
$$

where $k$ is the index on the four surrounding Eulerian nodes. The gradient of $S$ at node $(i, j)$ is

$$
\nabla S\left(\mathbf{x}_{p}\right)_{i, j}=S_{y_{j}} \frac{\partial S_{x_{i}}}{\partial x} \mathbf{e}_{\mathbf{x}}+S_{x_{i}} \frac{\partial S_{y_{j}}}{\partial y} \mathbf{e}_{\mathbf{y}}
$$

For a Cartesian grid, $\frac{\partial S_{x_{i}}}{\partial x}=-\frac{T_{i+1 / 2}}{\delta x_{i+1 / 2}}$ and $\frac{\partial S_{y_{j}}}{\partial y}=-\frac{T_{j+1 / 2}}{\delta y_{j+1 / 2}}$, where $\delta x_{i+1 / 2}=\left(x_{i+1}-x_{i}\right)$ and $\delta y_{j+1 / 2}=\left(y_{j+1}-y_{j}\right)$ are distances between cell centers. The four gradients of $S$, with support by the Eulerian nodes shown in Figure 2, are

$$
\nabla S\left(\mathbf{x}_{p}\right)_{i, j}=-\frac{S\left(\mathbf{x}_{p}\right)_{i+1 / 2, j}^{T S}}{\delta x_{i+1 / 2}} \mathbf{e}_{\mathbf{x}}-\frac{S\left(\mathbf{x}_{p}\right)_{i, j+1 / 2}^{T S}}{\delta y_{j+1 / 2}} \mathbf{e}_{\mathbf{y}}
$$




$$
\begin{gathered}
\nabla S\left(\mathbf{x}_{p}\right)_{i+1, j}=\frac{S\left(\mathbf{x}_{p}\right)_{i+1 / 2, j}^{T S}}{\delta x_{i+1 / 2}} \mathbf{e}_{\mathbf{x}}-\frac{S\left(\mathbf{x}_{p}\right)_{i, j+1 / 2}^{T S}}{\delta y_{j+1 / 2}} \mathbf{e}_{\mathbf{y}} \\
\nabla S\left(\mathbf{x}_{p}\right)_{i+1, j+1}=\frac{S\left(\mathbf{x}_{p}\right)_{i+1 / 2, j}^{T S}}{\delta x_{i+1 / 2}} \mathbf{e}_{\mathbf{x}}+\frac{S\left(\mathbf{x}_{p}\right)_{i, j+1 / 2}^{T S}}{\delta y_{j+1 / 2}} \mathbf{e}_{\mathbf{y}} \\
\nabla S\left(\mathbf{x}_{p}\right)_{i, j+1}=-\frac{S\left(\mathbf{x}_{p}\right)_{i+1 / 2, j}^{T S}}{\delta x_{i+1 / 2}} \mathbf{e}_{\mathbf{x}}+\frac{S\left(\mathbf{x}_{p}\right)_{i, j+1 / 2}^{T S}}{\delta y_{j+1 / 2}} \mathbf{e}_{\mathbf{y}}
\end{gathered}
$$

Equation (28) expanded in terms of its four interpolation function is

$$
\nabla \psi_{\mathrm{p}}=\nabla S\left(\mathbf{x}_{\mathrm{p}}\right)_{\mathrm{i}, \mathrm{j}} \psi_{\mathrm{i}, \mathrm{j}}+\nabla \mathrm{S}\left(\mathbf{x}_{\mathrm{p}}\right)_{\mathrm{i}+1, \mathrm{j}} \psi_{\mathrm{i}+1, j}+\nabla S\left(\mathbf{x}_{\mathrm{p}}\right)_{\mathrm{i}+1, \mathrm{j}+1} \psi_{\mathrm{i}+1, \mathrm{j}+1}+\nabla S\left(\mathbf{x}_{\mathrm{p}}\right)_{\mathrm{i}, \mathrm{j}+1} \psi_{\mathrm{i}, \mathrm{j}+1}
$$




\section{SOLUTION METHOD}

The governing equations for fluid and particles are solved on the computer. A computational particle method is used to solve for the particle distribution in lieu of direct solution of the Liouville equation. Particle properties are mapped to and from the grid using interpolation functions. The incompressible, two-dimensional continuum equations are solved using a finite volume method with a staggered rectangular grid and a pressure correction scheme. The numerical method implicitly couples phases through the interphase momentum transfer.

The numerical scheme uses an implicit calculation to overcome signal-wave limits on the computational time step. If the solution scheme was explicit, the numerical time step would be limited by the Courant-Friedrichs-Lewy condition and especially by stress waves from the interparticle stress (Gidaspow 1986). In two dimensions, the implicit solution gives four sets of equations containing two velocities, pressure and particle volume fraction. A set of equations is solved for one variable while holding the other variables fixed. Coupling between implicit variables is introduced by iterative solution of the sets of equations. As will be discussed later, the particle volume fraction needs to be solved in continuum form while iterating in the implicit loop.

The implicit numerical scheme with implicit coupling of interphase momentum between particles and fluid produces many parameters for each set of equations. For example, the twodimensional momentum equation incorporates nine velocities, six pressures, and six volume fraction variables. Because of the large number of parameters, each term is treated separately and its contribution is added to a variable's coefficient. Thus, the numerical implementation is straightforward. The following sections describe the numerical approximation to the governing equations.

\section{PARTICLE EQUATIONS FINITE DIFFERENCE APPROXIMATION}

Particles are grouped into computational parcels each containing $N_{p}$ particles with identical mass density, $\rho_{p}$, volume, $V_{p}$, and velocity, $\mathbf{u}_{p}$, located at position, $\mathbf{x}_{p}$. The Liouville equation (5) is the mathematical expression of conservation of particle numbers in volumes moving along dynamic trajectories in particle phase space. Thus the number of particles $N_{p}$ associated with a parcel is constant in time. Because there is no mass exchange between particles, a particle's mass, $m_{p}$, is also constant. Parcel positions are updated by

$$
\mathbf{x}_{p}^{n+1}=\mathbf{x}_{p}^{n}+\Delta t \mathbf{u}_{p}^{n+1}
$$

and the particle velocity is updated from integration of equation (6).

$$
\frac{\mathbf{u}_{p}^{n+1}-\mathbf{u}_{p}^{n}}{\Delta t}=D_{p}\left(\mathbf{u}_{g, p}^{n+1}-\mathbf{u}_{p}^{n+1}\right)-\frac{1}{\rho_{p}} \nabla p_{p}^{n+1}-\frac{1}{\rho_{p} \theta_{p}} \nabla \tau_{p}^{n+1}+\mathbf{g}
$$

where $\mathbf{u}_{g, p}^{n+1}$ is the interpolated implicit fluid velocity at the particle location, $\nabla p_{p}^{n+1}$ is the interpolated implicit pressure gradient at the particle location, $\nabla \tau_{p}^{n+1}$ is the interpolated implicit particle stress gradient at the particle location, $g$ is gravity acceleration, and $D_{p}$ is the drag coefficient. 
Solving equation (33) for $\mathbf{u}_{p}^{n+1}$ gives

$$
\mathbf{u}_{p}^{n+1}=\frac{\mathbf{u}_{p}^{n}+\Delta t\left[D_{p} \mathbf{u}_{g, p}^{n+1}-\frac{1}{\rho_{p}} \nabla p_{p}^{n+1}-\frac{1}{\rho_{p} \theta_{p}} \nabla \tau_{p}^{n+1}+\mathbf{g}\right]}{1+\Delta t D_{p}}
$$

where $\mathbf{u}_{g, p}^{n+1}$ is evaluated from equation (24) or (27) and $\nabla p_{p}^{n+1}$ and $\nabla \tau_{p}^{n+1}$ are evaluated from equation (31).

\section{EULERIAN DIFFERENCE APPROXIMATIONS}

The numerical scheme uses a finite volume method with staggered scalar and momentum nodes. The two-dimensional finite difference approximation uses a rectangular grid or cylindrical where scalar variables, pressure and volume fraction, are defined at cell centers and velocities are defined at volume surfaces staggered between cell centers. Finite volume approximations are described for the momentum equations, pressure equation (which is derived from volume continuity), and particle volume fraction equation in Cartesian coordinates.

\section{A. Momentum equation}

The finite volume approximation is described for the u-momentum equation, and a similar formulation is used for the v-momentum equation. The method is described in steps with coefficients on velocities, pressure and particle volume fraction added to coefficients from previous steps, gradually building a final algebraic equation.

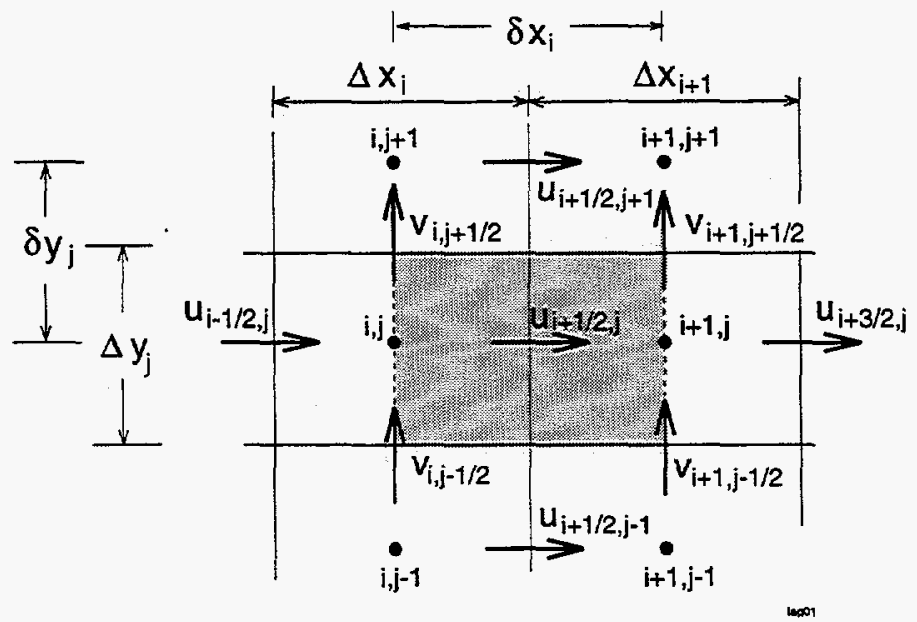

Figure 5. Momentum finite difference volume for $\mathrm{x}$-vector component.

\section{Time, convection, and viscous diffusion terms}

A conservation balance of u-momentum given by equation (2) is formed by integration over a momentum control volume with four neighbors. Figure 5 shows the Cartesian nodalization. Collecting terms associated with the five $u$-velocity variables gives the algebraic equation at $u$ momentum node $(i+1 / 2, j)$ : 


$$
\begin{aligned}
& c_{u_{c c}}\left(u_{g}\right)_{i+1 / 2, j}^{n+1}=c_{u_{r r}}\left(u_{g}\right)_{i+3 / 2, j}^{n+1}+c_{u l l}\left(u_{g}\right)_{i-1 / 2, j}^{n+1}+c_{u_{t t}}\left(u_{g}\right)_{i+1 / 2, j+1}^{n+1} \\
& +c_{u_{b b}}\left(u_{g}\right)_{i+1 / 2, j-1}^{n+1}+a_{u l l} p_{i, j}^{n+1}+a_{u_{r r}} p_{i+1, j}^{n+1}+\delta x \Delta y\left(F_{x}\right)_{i+1 / 2, j}^{n+1}+C_{u}^{\prime} .
\end{aligned}
$$

Coefficients are associated with the $u_{g_{i+1 / 2}}^{n+1}$ velocity, and $c, l, r, b$ and $t$ denote center, left, right, bottom and top, respectively. The contribution of the interphase momentum transfer, $F_{x}$, is given later. The diagonal coefficient $c_{u_{c c}}$ contains contributions from the time, convection and diffusion terms, and the neighbor velocity coefficients are from integration of convection and diffusion terms. The upwind convective velocity coefficient with diffusion for the left neighbor velocity, $u_{g_{i-1 / 2, j}}$ in Cartesian coordinates, is

$$
c_{u l l}=D_{i, j}+\max \left(0, f_{i, j}\right)
$$

where the flux and diffusion terms are

$$
f_{i, j}=\rho_{g}\left(\theta_{g} u_{g}\right)_{i, j}^{n} \Delta y_{j} \quad D_{i, j}=\frac{\left(\theta_{g} \mu\right)_{i, j}^{n} \Delta y_{j}}{\delta x_{i}}
$$

The max function specifies the maximum value of the two arguments. The cell center velocities in the flux term are taken as the average of the adjacent face center velocities, e.g.,

$$
\left(\theta_{g} u_{g}\right)_{i, j}^{n}=\frac{\left(\theta_{g} u_{g}\right)_{i-1 / 2, j}^{n}+\left(\theta_{g} u_{g}\right)_{i+1 / 2, j}^{n}}{2}
$$

and similarly for other cell center velocities.

The pressure coefficients in Cartesian coordinates are

$$
a_{u_{l l}}=\Delta y \quad a_{u_{r r}}=-\Delta y
$$

The Appendix lists all coefficients and constants in the linear algabraic momentum equations.

\section{Interphase momentum transfer}

Up to this point, a momentum equation contains five velocities and two pressures. An implicit-coupled interphase momentum transfer gives additional terms and contributes to existing terms. The implicit interphase momentum transfer is

$$
\mathbf{F}_{i+1 / 2, j}^{n+1}=\frac{1}{V} \sum_{i+1 / 2, j} S_{p}\left[D_{p}\left(\mathbf{u}_{g, p}^{n+1}-\mathbf{u}_{p}^{n+1}\right)-\frac{1}{\rho_{p}} \nabla p_{p}^{n+1}\right] N_{p} m_{p},
$$


where $m_{p}$ is the mass of a particle. If a $T$ or $T S$ interpolation is used, the fluid velocity at particle position $\mathbf{x}_{p}$ is from equation (24). If $S$ interpolation (bilinear interpolation) is used, the fluid velocity at particle position $\mathbf{x}_{p}$ is from equation (27). The particle pressure gradient is from equation (31).

The particle velocity, given by equation (34), is substituted into the $\mathbf{F}$ equation, and the $\mathbf{F}$ equation is subsequently substituted into the fluid momentum equation (2). The resulting discretized fluid momentum equation is

$$
\begin{gathered}
=\frac{V_{\zeta \backslash / 2} \rho_{g}\left(\theta_{g} \mathbf{u}_{g}\right)_{\zeta 1 / 2}^{n+1}}{\Delta t}+\operatorname{conv}_{\zeta 1 / 2}^{n+1} \\
=\frac{V_{\zeta 1 / 2} \rho_{g}\left(\theta_{g} \mathbf{u}_{g}\right)_{\zeta 1 / 2}^{n}}{\Delta t}-V_{\zeta 1 / 2} \nabla_{p}^{n+1}+\operatorname{diffus}_{\zeta 1 / 2}^{n+1}+V_{\zeta 1 / 2} \rho_{g} \theta_{g \zeta 1 / 2}^{n} \mathbf{g} \\
-\sum_{p} \frac{m_{p} N_{p} S_{\zeta 1 / 2}\left(\mathbf{x}_{p}\right) D_{p}}{\left(1+D_{p} \Delta t\right)} \sum_{\zeta 1 / 2} S_{\zeta 1 / 2}\left(\mathbf{x}_{p}\right)\left(\mathbf{u}_{g}\right)_{\zeta 1 / 2}^{n+1} \\
+\sum_{\cdot p} \frac{m_{p} N_{p} S_{\zeta 1 / 2}\left(\mathbf{x}_{p}\right)}{\left(1+D_{p} \Delta t\right) \rho_{p}} \sum_{\zeta} \nabla S_{\zeta}\left(\mathbf{x}_{p}\right) p_{\zeta}^{n+1} \\
+\sum_{p} \frac{m_{p} N_{p} S_{\zeta 1 / 2}\left(\mathbf{x}_{p}\right) D_{p}}{\left(1+D_{p} \Delta t\right)}\left(\mathbf{u}_{p}^{n}+\Delta t \mathbf{g}\right) \\
-\sum_{p} \frac{m_{p} N_{p} S_{\zeta 1 / 2}\left(\mathbf{x}_{p}\right) \Delta t D_{p}}{\left(1+D_{p} \Delta t\right) \rho_{p} \theta_{p}^{n}} \sum_{\zeta} \nabla S_{\zeta}\left(\mathbf{x}_{p}\right) \tau_{\zeta}^{n+1} .
\end{gathered}
$$

The summation is over all particles. Abbreviated subscripts are used where $\zeta_{1 / 2}$ is the face node, $\zeta$ is the cell center node, $S_{\zeta I / 2}$ is the face interpolation functions, and $S_{\zeta}$ is the cell center interpolation function. The first two lines of equation (41) contain the transient, convective, and diffusive terms which were previously discussed.

The interphase drag adds to the existing momentum equation coefficients and generates additional velocity, pressure and particle volume fraction terms. Consider the pressure term in equation (41),

$$
\sum_{p} \frac{m_{p} N_{p} S_{\zeta 1 / 2}\left(\mathbf{x}_{p}\right)}{\left(1+D_{p} \Delta t\right) \rho_{p}} \sum_{\zeta} \nabla S_{\zeta}\left(\mathbf{x}_{p}\right) p_{\zeta}^{n+1}
$$

The pressure gradient interpolated to particle location $\mathbf{x}_{p}$ (see Figure 2) is

$$
\sum_{\zeta} \nabla S_{\zeta}\left(\mathbf{x}_{p}\right) p_{\zeta}^{n+1}=\nabla S_{i, j} p_{i, j}^{n+1}+\nabla S_{i+1, j} p_{i+1, j}^{n+1}+\nabla S_{i+1, j+1} p_{i+1, j+1}^{n+1}+\nabla S_{i, j+1} p_{i, j+1}^{n+1}
$$


The $\mathrm{x}$-directed pressure force from a single parcel of particles is distributed between face centers $(i+1 / 2, j)$ and $(i+1 / 2, j+1)$. A TS interpolation (two-neighbor interpolation) function is used to interpolate the particle pressure gradient back to the Eulerian grid. The pressure gradient on $\mathrm{x}$ momentum cell $(i+1 / 2, j)$ from a particle is

$$
\begin{aligned}
\nabla p_{p_{i+1 / 2, j}}=\frac{N_{p} m_{p}}{\left(1+D_{p} \Delta t\right) \rho_{p}} S_{i+1 / 2, j}^{T S}\left(\nabla S_{i, j} p_{i, j}^{n+1}+\nabla S_{i+1, j} p_{i+1, j}^{n+1}\right. \\
\left.+\nabla S_{i+1, j+1} p_{i+1, j+1}^{n+1}+\nabla S_{i, j+1} p_{i, j+1}^{n+1}\right) \cdot \mathbf{e}_{x}
\end{aligned}
$$

and the particle pressure gradient contribution to $\mathrm{x}$-momentum cell $(i+1 / 2, j+1)$ is

$$
\begin{aligned}
\nabla p_{p_{i+1 / 2, j+1}}=\frac{N_{p} m_{p}}{\left(1+D_{p} \Delta t\right) \rho_{p}} S_{i+1 / 2, j+1}^{T S}\left(\nabla S_{i, j} p_{i, j}^{n+1}+\nabla S_{i+1, j} p_{i+1, j}^{n+1}\right. \\
\left.+\nabla S_{i+1, j+1} p_{i+1, j+1}^{n+1}+\nabla S_{i, j+1} p_{i, j+1}^{n+1}\right) \cdot \mathbf{e}_{x} .
\end{aligned}
$$

The particle pressure contributions at each node are added to existing pressure coefficients in the discretized momentum equation. For face node $(i+1 / 2, j)$, the resulting coefficients from pressure for a single parcel of particles are

$$
\begin{gathered}
a_{u_{l l}}=a_{u_{l l}}^{\prime}+\frac{N_{p} m_{p}}{\left(1+D_{p} \Delta t\right) \rho_{p}} S_{i+1 / 2, j+1}^{T S} \nabla S_{x_{i, j}} \\
a_{u_{r r}}=a_{u_{r r}}^{\prime}+\frac{N_{p} m_{p}}{\left(1+D_{p} \Delta t\right) \rho_{p}} S_{i+1 / 2, j+1}^{T S} \nabla S_{x_{i+1, j}} \\
a_{u_{t r}}=a_{u_{t r}}^{\prime}+\frac{N_{p} m_{p}}{\left(1+D_{p} \Delta t\right) \rho_{p}} S_{i+1 / 2, j+1}^{T S} \nabla S_{x_{i+1, j+1}} \\
a_{u_{t l}}=a_{u_{t l}}^{\prime}+\frac{N_{p} m_{p}}{\left(1+D_{p} \Delta t\right) \rho_{p}} S_{i+1 / 2, j+1}^{T S} \nabla S_{x_{i, j+1}}
\end{gathered}
$$

where $a_{u}^{\prime}$ is the existing coefficient, and $\nabla S_{x}$ is the x-component of the gradient. Additional pressure coefficients $a_{u_{t r}}$ and $a_{u_{t l}}$ are created from interphase momentum transfer. Similar coefficients are modified for node $(i+1 / 2, j+1)$, with new coefficients $a_{u_{b r}}$ and $a_{u_{b l}}$ being generated. The result is an increase from two pressure terms to six pressure terms in the u-momentum equation and similarly for the v-momentum equation. The total particle pressure on the fluid velocity is obtained by summing the contribution from all particles. 
Similar to the pressure example above, the other terms in equation (41) are decomposed into coefficients times their respective variables. The resulting u-momentum equation is

$$
\begin{gathered}
c_{u_{c c}}\left(u_{g}\right)_{i+1 / 2, j}^{n+1}=C_{u_{i+1 / 2, j}} \\
+c_{u_{r r}}\left(u_{g}\right)_{i+3 / 2, j}^{n+1}+c_{u_{l}}\left(u_{g}\right)_{i-1 / 2, j}^{n+1}+c_{u_{t l}}\left(u_{g}\right)_{i+1 / 2, j+1}^{n+1}+c_{u_{b b}}\left(u_{g}\right)_{i+1 / 2, j-1}^{n+1} \\
+c_{u_{t r}}\left(u_{g}\right)_{i+3 / 2, j+1}^{n+1}+c_{u_{l l}}\left(u_{g}\right)_{i-1 / 2, j+1}^{n+1}+c_{u_{b r}}\left(u_{g}\right)_{i+3 / 2, j-1}^{n+1}+c_{u_{b l}}\left(u_{g}\right)_{i-1 / 2, j-1}^{n+1} \\
+a_{u_{l l}}(p)_{i, j}^{n+1}+a_{u_{r r}}(p)_{i+1, j}^{n+1}+a_{u_{b l}}(p)_{i, j-1}^{n+1}+a_{u_{b r}}(p)_{i+1, j-1}^{n+1}+a_{u_{t l}}(p)_{i, j+1}^{n+1}+a_{u_{t r}}(p)_{i+1, j+1}^{n+1} \\
+b_{u l l}\left(\theta_{p}\right)_{i, j}^{n+1}+b_{u_{r r}}\left(\theta_{p}\right)_{i+1, j}^{n+1}+b_{u_{b l}}\left(\theta_{p}\right)_{i, j-1}^{n+1}+b_{u_{b r}}\left(\theta_{p}\right)_{i+1, j-1}^{n+1}+b_{u_{t l}}\left(\theta_{p}\right)_{i, j+1}^{n+1}+b_{u t r}\left(\theta_{p}\right)_{i+1, j+1}^{n+1},
\end{gathered}
$$

and the v-momentum equation is

$$
\begin{gathered}
c_{v_{c c}}\left(v_{g}\right)_{i, j+1 / 2}^{n+1}=C_{v_{i, j+1 / 2}} \\
+c_{v_{r r}}\left(v_{g}\right)_{i+1, j+1 / 2}^{n+1}+c_{v_{l l}}\left(v_{g}\right)_{i-1, j+1 / 2}^{n+1}+c_{v_{t t}}\left(v_{g}\right)_{i, j+3 / 2}^{n+1}+c_{v_{b b}}\left(v_{g}\right)_{i, j-1 / 2}^{n+1} \\
+c_{v_{t r}}\left(v_{g}\right)_{i+1, j+3 / 2}^{n+1}+c_{v_{t l}}\left(v_{g}\right)_{i-1, j+3 / 2}^{n+1}+c_{v_{b r}}\left(v_{g}\right)_{i+1, j-1 / 2}^{n+1}+c_{v_{b l}}\left(v_{g}\right)_{i-1, j-1 / 2}^{n+1} \\
+a_{v_{b b}}(p)_{i, j}^{n+1}+a_{v_{t t}}(p)_{i, j+1}^{n+1}+a_{v_{b l}}(p)_{i-1, j}^{n+1}+a_{v_{b r}}(p)_{i+1, j}^{n+1}+a_{v_{t l}}(p)_{i-1, j+1}^{n+1}+a_{v_{t r}}(p)_{i+1, j+1}^{n+1} \\
+b_{v_{b b}}\left(\theta_{p}\right)_{i, j}^{n+1}+b_{v_{t t}}\left(\theta_{p}\right)_{i, j+1}^{n+1}+b_{v_{b l}}\left(\theta_{p}\right)_{i-1, j}^{n+1}+b_{v_{b r}}\left(\theta_{p}\right)_{i+1, j}^{n+1}+b_{v_{t l}}\left(\theta_{p}\right)_{i-1, j+1}^{n+1}+b_{v_{t r}}\left(\theta_{p}\right)_{i+1, j+1}^{n+1} .
\end{gathered}
$$

The momentum equations contain the particle volume fraction, $\theta_{p}$, associated with the interparticle stress. The interparticle stress has been linearized as a function of the implicit particle volume fraction by retaining linear terms in the Taylor series expansion of equation (11) about the old-time particle volume fraction:

where

$$
\tau_{i, j}^{n+1}=c_{\tau 1_{i, j}} \theta_{p, i, j}^{n+1}+c_{\tau 2_{i, j}}
$$

$$
\begin{gathered}
c_{\tau 1_{i, j}}=\frac{P_{s}\left(\theta_{p_{i, j}}^{n}\right)_{i, j}^{\beta-1}}{\left(\theta_{c p}-\theta_{p_{i, j}}^{n}\right)^{2}}\left[\theta_{p_{i, j}}^{n}+\beta\left(\theta_{c p}-\theta_{p_{i, j}}^{n}\right)\right] \\
c_{\tau 2_{i, j}}=-\frac{P_{s}\left(\theta_{p_{i, j}}^{n}\right)_{i, j}^{\beta-1}}{\left(\theta_{c p}-\theta_{p_{i, j}}^{n}\right)^{2}}\left[\theta_{p_{i, j}}^{n}+(\beta-1)\left(\theta_{c p}-\theta_{p_{i, j}}^{n}\right)\right] \theta_{p_{i, j}}^{n} .
\end{gathered}
$$

The coefficients on particle volume fraction in the momentum equations (46) and (47) arise in a similar fashion as those described above for the pressure coefficients. The coefficients and constants are given in the Appendix. 


\section{PRESSURE EQUATION}

Following the usual practice for incompressible flow, a SIMPLE type algorithm is used in which solution of the momentum equations uses a guessed pressure field, $p^{\prime}$, to calculate velocities which are subsequently corrected by satisfying continuity (Patankar 1980). The velocity and pressure are $\mathbf{u}=\mathbf{u}^{\prime}+\delta \mathbf{u}$ and $p=p^{\prime}+\delta p$ where the velocity field $\mathbf{u}^{\prime}$ is solved from momentum equations (46) and (47) with guessed pressure field $p^{\prime}$. The $\delta \mathbf{u}$ and $\delta p$ correct the velocity and pressure fields to satisfy continuity.

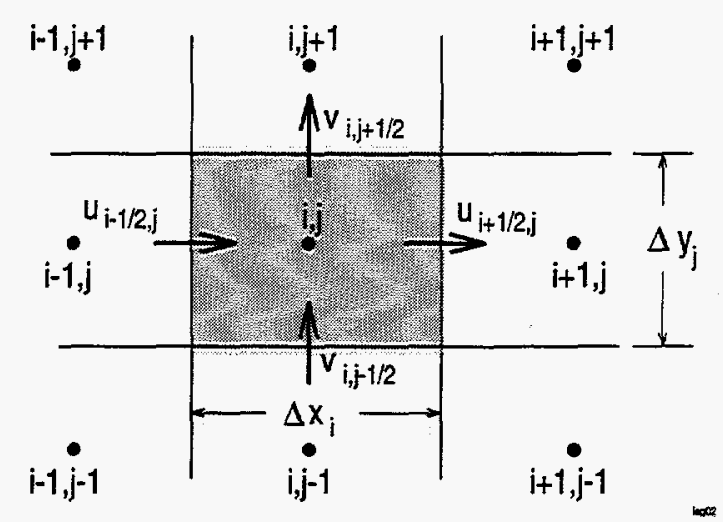

Figure 6. Continuity equation finite difference volume.

Figure 6 shows the control volume. The pressure equation is formed from substituting velocity equations (46) and (47) into the continuity equation (1), and ignoring $\delta \mathbf{u}$ and $\delta p$ associated with neighbor nodes. This forms a nine-point pressure stencil with nodes shown in Figure 7. The pressure equation is

$$
\begin{aligned}
& a_{p_{c c}} \delta p_{i, j}+a_{p l l} \delta p_{i-1, j}+a_{p_{r r_{i, j}}} \delta p_{i+1, j}+a_{p b b} \delta p_{i, j-1}+a_{p_{t t}, j} \delta p_{i, j+1} \\
& +a_{p b l} \delta p_{i-1, j-1}+a_{p_{b r}} \delta p_{i, j} p_{i+1, j-1}+a_{p_{t l}} \delta p_{i-1, j+1}+a_{p_{t r}} \delta p_{i+j} p_{i+1, j+1}=-e r r_{i, j} .
\end{aligned}
$$

The coefficients are given in the Appendix. The $e r r_{i, j}$ is the mass residual obtained from solving the continuity equation using the uncorrected velocity field. For Cartesian coordinates the continuity equation is

$$
\begin{gathered}
e r_{i, j}=\frac{\left(V \theta_{g}\right)_{i, j}^{n+1}-\left(V \theta_{g}\right)_{i, j}^{n}}{\Delta t}+\Delta y_{j}\left(u_{g}^{\prime} \theta_{g}\right)_{i+1 / 2, j}^{n+1}-\Delta y_{j}\left(u_{g}^{\prime} \theta_{g}\right)_{i-1 / 2, j}^{n+1} \\
+\Delta x_{i}\left(v_{g}^{\prime} \theta_{g}\right)_{i, j+1 / 2}^{n+1}-\Delta x_{i}\left(v_{g}^{\prime} \theta_{g}\right)_{i, j-1 / 2}^{n+1}
\end{gathered}
$$




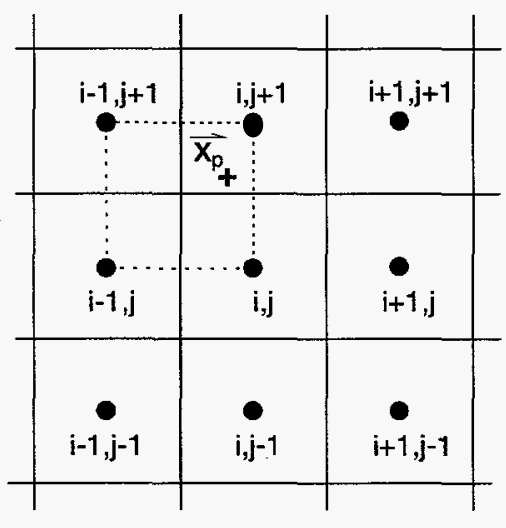

Figure 7 . The 9 point pressure stencil for the pressure equation.

In Eulerian fluid and particle methods, the volume fractions are calculated from the Eulerian continuity equations. In contrast, the MP-PIC Eulerian-Lagrangian method does not use an Eulerian continuity equation to calculate volume fractions, but instead, both particle and fluid volume fractions come from interpolation of particles to the Eulerian grid as given by

$$
\theta_{p_{i, j}}=\frac{1}{V_{i, j}} \sum_{p} \frac{m_{p} N_{p}}{\rho_{p}} S_{i, j}\left(\mathbf{x}_{p}\right) .
$$

The fluid volume fraction is calculated from conservation of volume where the fluid and particle volumes sum to unity, $\theta_{g_{i, j}}=1-\theta_{p_{i, j}}$.

\section{A. Relaxation factors}

During the pressure correction step the particle volume fraction is held constant but is coupled to the particle volume fraction solution through implicit iterations. Because the time rate of change of volume fraction is fixed during the pressure correction step of the implicit iteration loop, it was found that relaxation factors within the iteration loop could give a smoother, faster converging solution. Typical values of relaxation ranged from 0.6 to 1 .

\section{PARTICLE VOLUME FRACTION ON THE EULERIAN GRID}

It is not prudent to update the volume fraction within an iteration loop where particles are continuously summed as given by equation (22). Andrews and O'Rourke (1995) presented a method of approximating the new-time volume fraction on the Eulerian grid using explicit coefficients. In the method, a time step begins by estimating new particle positions using the old particle velocities:

$$
\widetilde{\mathbf{x}}_{p}=\mathbf{x}_{p}^{n}+\mathbf{u}_{p}^{n} \Delta t
$$

The explicit void fraction at $\widetilde{\mathbf{x}}_{p}$ is 


$$
\tilde{\theta}_{p_{i, j}}=\frac{1}{V_{i, j}} \sum_{p} \frac{m_{p} N_{p}}{\rho_{p}} S_{i, j}\left(\tilde{\mathbf{x}}_{p}\right)
$$

The volume fraction at the end of the time step is estimated using a Taylor series expansion of equation (52) about the intermediate particle position $\widetilde{\mathbf{x}}_{p}$ and retaining linear terms:

$$
\theta_{p_{i, j}}^{n+1} \approx \tilde{\theta}_{p_{i, j}}+\frac{1}{V_{i, j}} \sum_{p} \frac{m_{p} N_{p}}{\rho_{p}} \nabla S_{i, j}\left(\tilde{\mathbf{x}}_{p}\right) \cdot\left(\mathbf{x}_{p}^{n+1}-\widetilde{\mathbf{x}}_{p}\right) .
$$

Substituting $\mathbf{x}_{p}^{n+1}$ from equation (32), $\widetilde{\mathbf{x}}_{p}$ from equation (53), and $\mathbf{u}_{p}^{n+1}$ from equation (34) into equation (55) gives an approximate value for the new-time volume fraction:

$$
\begin{aligned}
\theta_{p \zeta}^{n+1}= & \tilde{\theta}_{p \zeta}+\frac{\Delta t^{2}}{V_{\zeta}} \sum_{p} \frac{m_{p} N_{p} D_{p}}{\rho_{p}\left(1+D_{p} \Delta t\right)} \nabla S_{\zeta}\left(\tilde{\mathbf{x}}_{p}\right) \cdot \mathbf{u}_{g, p}^{n+1} \\
& +\frac{\Delta t^{2}}{V_{\zeta}} \sum_{p} \frac{m_{p} N_{p}}{\rho_{p}\left(1+D_{p} \Delta t\right)} \nabla S_{\zeta}\left(\tilde{\mathbf{x}}_{p}\right) \cdot \mathbf{g} \\
& -\frac{\Delta t^{2}}{V_{\zeta}} \sum_{p} \frac{m_{p} N_{p}}{\rho_{p}\left(1+D_{p} \Delta t\right)} D_{p} \nabla S_{\zeta}\left(\tilde{\mathbf{x}}_{p}\right) \cdot \mathbf{u}_{p}^{n} \\
& -\frac{\Delta t^{2}}{V_{\zeta}} \sum_{p} \frac{m_{p} N_{p}}{\rho_{p}{ }^{2}\left(1+D_{p} \Delta t\right)} \nabla S_{\zeta}\left(\tilde{\mathbf{x}}_{p}\right) \cdot \nabla p_{p}^{n+1} \\
- & \frac{\Delta t^{2}}{V_{\zeta}} \sum_{p} \frac{m_{p} N_{p}}{\rho_{p}{ }^{2} \theta_{p}^{n}\left(1+D_{p} \Delta t\right)} \nabla S_{\zeta}\left(\tilde{\mathbf{x}}_{p}\right) \cdot \nabla \tau_{p}^{n+1} .
\end{aligned}
$$

Abbreviated subscripts are used where $\zeta$ is the cell center node, $\nabla S_{\zeta}$ is the gradient of the interpolation function given by equation (29), and $\nabla p_{p}^{n+1}$ and $\nabla \tau_{p}^{n+1}$ are given by equation (31) for pressure and interparticle stress, respectively. The fluid velocity is from a TS interpolation (twoneighbor) or $T$ interpolation (closest-neighbor). A bilinear interpolation would produce a 12 point stencil for u-velocity and 12 point stencil for v-velocity. By using the $T S$ or $T$ interpolation, the $\mathrm{u}$ and $\mathrm{v}$ stencils are reduced to six.

The implicit particle void fraction is rewritten in terms of coefficients:

$$
\begin{aligned}
& b_{\theta_{c c}} \theta_{i, j}^{n+1}=a_{\theta_{c c}} p_{i, j}^{n+1}+ a_{\theta_{l l}} p_{i-1, j}^{n+1}+a_{\theta_{r r}} p_{i+1, j}^{n+1}+a_{\theta_{b b}} p_{i, j-1}^{n+1} a_{\theta_{t t}} p_{i, j+1}^{n+1}+a_{\theta_{b l}} p_{i-1, j-1}^{n+1} \\
&+a_{\theta_{b r}} p_{i+1, j-1}^{n+1}+a_{\theta_{t l}} p_{i-1, j+1}^{n+1}+a_{\theta_{t r}} p_{i+1, j+1}^{n+1} \\
&+ b_{\theta_{l}} \theta_{i-1, j}^{n+1}+b_{\theta_{r r}} \theta_{i+1, j}^{n+1}+b_{\theta_{b b}} \theta_{i, j-1}^{n+1} b_{\theta_{t t}} \theta_{i, j+1}^{n+1}+b_{\theta_{b l}} \theta_{i-1, j-1}^{n+1} \\
&+b_{\theta_{b r}} \theta_{i+1, j-1}^{n+1}+b_{\theta_{t l}} \theta_{i-1, j+1}^{n+1}+b_{\theta_{t r}} \theta_{i+1, j+1}^{n+1} \\
&+ g_{\theta_{l l}} u_{g, i-1 / 2, j}^{n+1} \\
&+g_{\theta_{r r}} u_{g, i+1 / 2, j}^{n+1}+g_{\theta_{b l}} u_{g, i-1 / 2, j-1}^{n+1}+g_{\theta_{b r}} u_{g, i+1 / 2, j-1}^{n+1}
\end{aligned}
$$




$$
\begin{gathered}
+g_{\theta_{t l}} u_{g, i-1 / 2, j+1}^{n+1}+g_{\theta_{t r}} u_{g, i+1 / 2, j+1}^{n+1} \\
+h_{\theta_{b b}} v_{g, i, j-1 / 2}^{n+1}+h_{\theta_{t t}} v_{g, i, j+1 / 2}^{n+1}+h_{\theta_{b l}} v_{g, i-1, j-1 / 2}^{n+1}+h_{\theta_{b r}} v_{g, i+1, j-1 / 2}^{n+1} \\
+h_{\theta_{t l}} v_{g, i-1, j+1 / 2}^{n+1}+h_{\theta_{t r}} v_{g, i+1, j+1 / 2}^{n+1}+c_{\theta}=0 .
\end{gathered}
$$

The coefficients are for the cell center node $(i, j)$, and the interparticle stress has been linearized as a function of the implicit particle volume fraction by equation (48). Coefficients are given in the Appendix.

\section{DISCUSSION OF NUMERICAL FORMULATION}

\section{A. Interpolation method}

Three methods of interpolation were presented earlier. A difference was found in the results when using the different methods. A bilinear interpolation is always used for interpolating continuum properties from the Eulerian grid to particle positions. In the interphase momentum transfer, particle properties are mapped to the Eulerian grid using either bilinear interpolation, TS interpolation or the $T$ interpolation. The justification for the interpolation methods are given below.

\section{Interpolation of the continuum properties to particle positions}

The use of the $T S$ interpolation (two-neighbor) or $T$ interpolation (nearest-neighbor) of the fluid velocity to particle positions appears logical in that the interpolated fluid velocities and particles lie between adjacent cell centers where pressure and interparticle stress gradients are defined. However, it was found in the Lagrangian particle calculations that a bilinear interpolation ( $S$ interpolation) of fluid velocity to particle positions was required to give an acceptably smooth distribution of particles. The left figure in Figure 8 shows a Rayleigh-Taylor problem using the TS interpolation of fluid velocity in solving the particle velocity, and the right figure in Figure 8 shows the same problem using the bilinear interpolation. The TS interpolation produced clumping and the bilinear interpolation gave more uniformly distributed particles.

In the current numerical formulation, the aerodynamic drag is the only mechanism which can be interpolated to give a unique, position dependent force on a particle. The other driving forces on a particle are a uniform gravity field, and gradients. The gradients impose the fixed granularity of the Eulerian grid on particles where all particles residing between cell centers are driven by the same gradient. The Lagrangian particles require a high fidelity in local properties.

Using the $T S$ interpolation, the fluid velocity is interpolated to a particle position in a single direction only. This produces horizontal lines of constant u-velocity and vertical lines of constant v-velocity. Further, the interpolated fluid velocities step change in value as particles cross cell center boundaries. The $T$ interpolation suffers the same problem. The result is clumping of particles. The bilinear interpolation provides a unique fluid velocity for each particle position which smoothly transitions between interpolation cells, giving a smoother distribution of particles. 


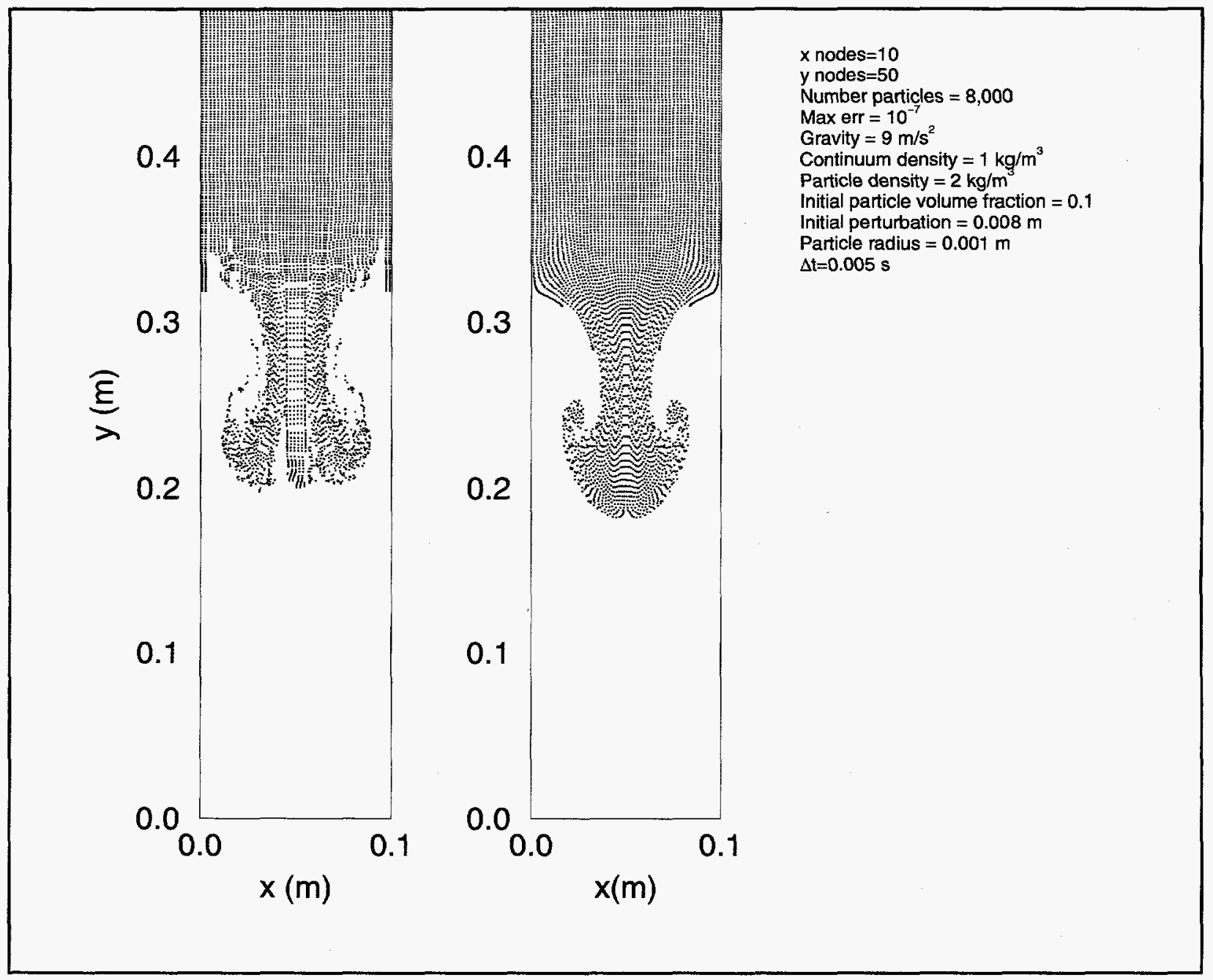

Figure 8. Rayleigh-Taylor calculation. Left: uses TS interpolation function from Eulerian grid to particle position. Right: uses bilinear interpolation function from Eulerian grid to particle position. 


\section{Interpolation of the particle properties to the Eulerian grid}

The Lagrangian particle calculations require fidelity of the bilinear interpolation of fluid properties to each particle position to obtain a smooth distribution of particles. On the other hand, the $T$ interpolation of particle properties to the Eulerian grid may be justified and has been previously used by Andrews and O'Rourke (1995). The Eulerian calculation grid represents average properties over finite volumes and does not require the bilinear interpolation of particle properties to a point on the Eulerian grid. It is sufficient to interpolate particle properties to a volume using a $T$ interpolation function. In the implicit Eulerian interphase momentum solution, a multiplication of bilinear and $T$ interpolation functions will arise. The Eulerian properties are bilinearly interpolated to each particle position, and then each particle property is interpolated back to the Eulerian grid using a $T$ interpolation function.

The $T$ interpolation of pressure and particle stress gradients from particles to the Eulerian grid produces a simpler numerical solution and eliminates the chance of a "checker-board" pressure field. In the pressure example from interphase momentum transfer discussed above, a bilinear interpolation from particles to a point on the Eulerian grid gives a 12 point pressure stencil compared to the 6 point stencil from a $T$ interpolation. The bilinear interpolation produces more coefficients and a more difficult boundary condition. Also with the 12 point pressure stencil, face velocities may be driven by alternate node pressures which can produce a checkerboard pressure.

\section{B. Calculated layering of particles in a quasi-steady fluidized bed}

The fluidized bed examples pointed to numerical implementation issues where forms of the numerical approximations can produce layers of particles clustered at unique levels during quasi-steady conditions. The numerical formulation that leads to particle clumping is presented with a suitable numerical solution.

It was initially observed that in the fluidized bed calculation, particles clustered at unique vertical levels. The bands of particles formed as the bed reached a quasi-steady condition. This clustering formed, in part, from a nearly constant fluid velocity and from all particles between grid nodes being driven by the same pressure gradient and interparticle stress gradient. Two examples are given where particles cluster into bands because of the numerical formulation.

A fluidized bed reaches a quasi-steady condition where the mean horizontal fluid velocity is zero and particle velocities are zero:

$$
\begin{array}{ll}
\bar{u}_{g}=0 & \bar{v}_{g}=v(y) \\
u_{p}=0 & v_{p}=0 .
\end{array}
$$

The force on particles is described by equation (6). For steady flow, the force balance is

$$
D_{p} v_{g, p}=\left[\frac{1}{\rho_{p}} \nabla p_{p}-\mathbf{g}+\frac{1}{\theta_{p} \rho_{p}} \nabla \tau_{p}\right] \cdot \mathbf{e}_{y} .
$$

Gravity is constant, and the gradients, $\nabla p_{p}$ and $\nabla \tau_{p}$, are constant for all particles within the nodes supporting $S$. 
In the first example, a $S^{T}$ or $S^{T S}$ interpolation is used to get the gas velocity, $v_{g, p}$, at a particle position. The gas velocity is constant for all particles between nodes. The drag coefficient, $D_{p}$, contains $\theta_{g, p}$, and the interparticle stress contains $\theta_{p}$. Volume fraction varies only in the vertical direction. For a typical case

$$
\theta_{p}=\left(\theta_{p_{j}}+\theta_{p_{j+1}}\right) / 2
$$

which is constant for all particles between nodes. The drag coefficient is

$$
D_{p}=C_{d} \frac{3}{8} \frac{\rho_{g}}{\rho_{p}} \frac{\left|v_{g}\right|}{r}
$$

For simplicity

$$
C_{d}=\frac{24}{\operatorname{Re}} \frac{1}{\theta_{g, p}} \quad \text { and } \quad \operatorname{Re}=\frac{2 \rho_{g}\left|v_{g}\right| r}{\mu_{g}}
$$

The fluid volume fraction is defined from an interpolation from the grid to the particle position:

$$
\theta_{g, p}=\left(\theta_{g_{j+1}}-\theta_{g_{j}}\right) \frac{\left(y_{p}-y_{j}\right)}{\Delta y}+\theta_{g_{j}} .
$$

Substituting equations (59) through (62) into equation (58) and solving for $y_{p}$ gives the vertical position where particles locate at steady condition

$$
\begin{gathered}
y_{p}=y_{j}+\left(\frac{c_{2}}{c_{1}}-\theta_{g_{j}}\right) \frac{\Delta y}{\left(\theta_{g_{j}+1}-\theta_{g_{j}}\right)} \\
c_{1}=\frac{\nabla p_{p}}{\rho_{p}}+\frac{\nabla \tau_{p}}{\rho_{p} \theta_{p}}-g \\
c_{2}=\frac{9}{2} \frac{\mu_{g} u_{g}}{\rho_{p} r^{2}} .
\end{gathered}
$$

The parameters $c 1$ and $c 2$ are constant within the domain of $S$, and so the vertical location of particles is governed by the fluid volume fraction, $\theta_{g j}$. Using the calculated volume fraction, the vertical position of particles can be predicted. Figure 9 shows the layering which results from mapping the particle volume fraction to particle position in the interphase drag term. The predicted vertical particle locations match the calculated particle locations. The particles are distributed both vertically and horizontally in the two-dimensional calculation, but only vertical locations are plotted in the figure ( $\mathrm{x}$ coordinate value set to a constant). Thus the one visible circle in the figure is in fact many particles stacked on top of each other. By removing the interpolation of volume fraction to particle position in the 
drag coefficient (constant volume fraction within nodes supporting $S$ ), calculated particle positions are distributed smoothly between grid nodes, as shown in Figure 9.

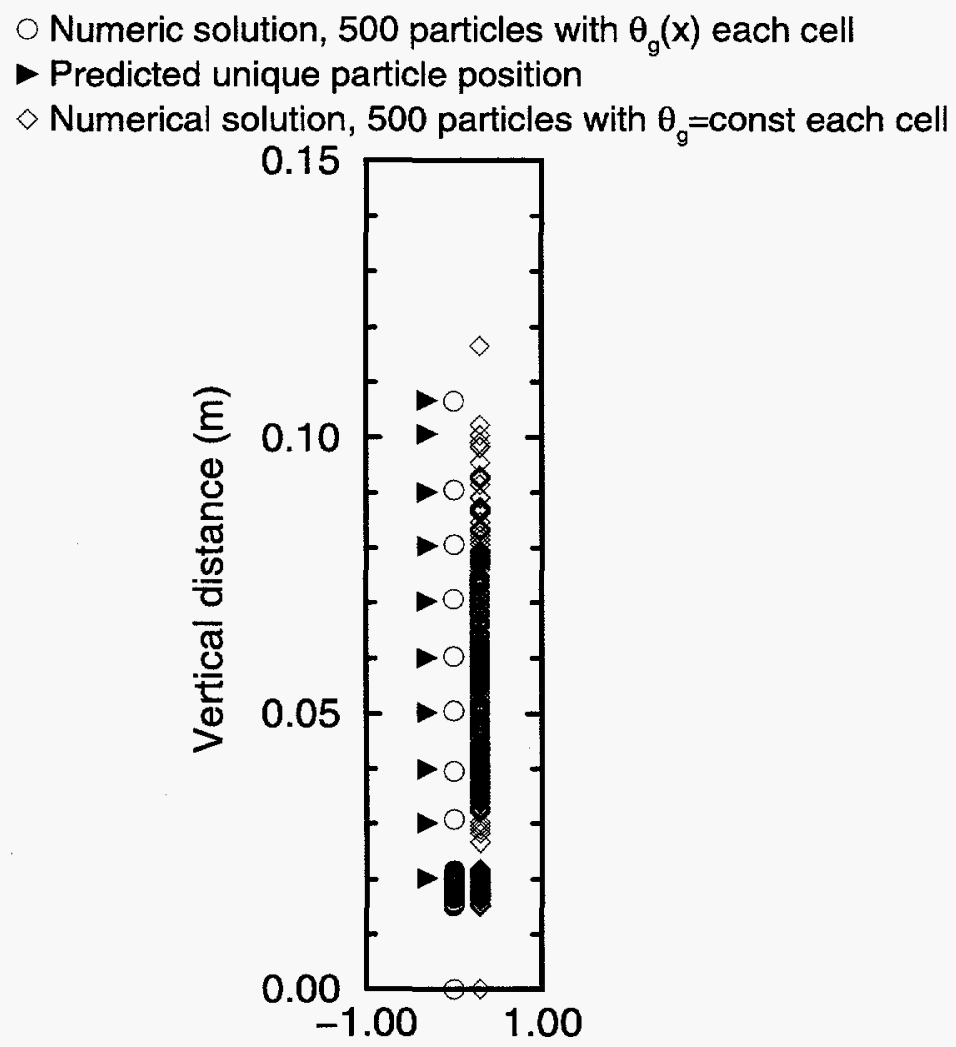

Figure 9. Unique particle position in a fluidized bed from $\theta_{g}$ interpolation to particle location.

It was previously determined that a $S^{T}$ or $S^{T S}$ interpolation of fluid velocity to a particle position gave clumping of particles in transient calculations, and it was deemed necessary to use a $S^{S}$ interpolation. However, even if the $S^{S}$ interpolation is used, bands of particles can still form if $D_{p}$ is constant between nodes. The fluid velocity at particle position $y_{p}$ from a $S^{S}$ interpolation is

$$
v_{g, p}=\left(v_{g_{j+1}}-v_{g_{j}}\right) \frac{\left(y_{p}-y_{j}\right)}{\Delta y}+v_{g_{j}} \text {. }
$$

If the fluid volume fraction used in $D_{p}$, which is defined in the previous example, is an average between nodes, particles will again cluster in bands. Similar to the previous example, knowing the gas velocity, the particle band positions are known.

The two examples illustrate considerations in formulation of the numerical scheme. Again, it is warranted to use a bilinear interpolation from the Eulerian grid to particle positions to get a smooth distribution of particles. The fluid velocities at a particle positions are always from a bilinear interpolation. The volume fraction in the drag correlation is also from a bilinear interpolation from the Eulerian grid. 


\section{NUMERICAL DIFFUSION}

The fluid-to-particle viscous force is modeled using the interphase momentum transfer term which has as its basis Stokes flow. There is no model for viscous-type particle-to-particle momentum transfer. The question arises whether the numerical formulation, which uses bilinear interpolation of neighbor velocities, introduces numerical diffusion through the interphase drag. To examine numerical diffusion, the interface drag is set to a large value, $D_{p}=10^{16} \mathrm{~s}^{-1}$. This forces the particle velocity to be close to the gas velocity at the particle position and eliminates momentum transfer from particles moving through the fluid. It can be reasoned that because the fluid interphase momentum equation (40) uses the same bilinear interpolation as used in the particle velocity equation (33), an explicit formulation gives zero interphase drag and thus no diffusion. To evaluate the implicit solution, the problem of flow between two flat plates is considered. The bottom plate is fixed and the top plate moves at twice the uniform inlet fluid velocity. The viscosity is set very small $\left(\mu_{\mathrm{g}}=10^{-18} \mathrm{~kg} / \mathrm{m}-\mathrm{s}\right)$ to give near inviscid flow but still maintain a no-slip boundary condition at the rigid walls. A uniform distribution of 0.01 volume fraction of particles is introduced near the entrance. The grid has 6 non-uniform cross channel nodes and 15 uniform axial nodes. The cross channel nodes are small near the boundary and larger in the channel center. Table 1 gives specifications of the problem.

Table 1. Particle flow between flat plates.

\begin{tabular}{lc} 
Number of parcels & 8,000 \\
Particle radius $(\mathrm{mm})$ & 0.001 \\
Particle density $\left(\mathrm{kg} / \mathrm{m}^{3}\right)$ & 35.4 \\
Fluid density $\left(\mathrm{kg} / \mathrm{m}^{3}\right)$ & 1 \\
$P_{s}(\mathrm{~Pa})$ & 0 \\
$\theta_{c p}$ & 0.7 \\
$\mathrm{x}$-gravity, y-gravity $\left(\mathrm{m} / \mathrm{s}^{2}\right)$ & 0,0 \\
Number x-cells (uniform $\Delta \mathrm{x})$ & 15 \\
Number y-cells & 6 \\
$\quad \Delta \mathrm{y}_{1}=0.00556 \mathrm{~m} \quad \Delta \mathrm{y}_{2}=0.01667 \mathrm{~m}$ & \\
$\quad \Delta \mathrm{y}_{3}=0.02778 \mathrm{~m} \quad \Delta \mathrm{y}_{4}=0.02778 \mathrm{~m}$ & \\
$\quad \Delta \mathrm{y}_{5}=0.01667 \mathrm{~m} \quad \Delta \mathrm{y}_{6}=0.00556 \mathrm{~m}$ & \\
$\mathrm{x}$-domain (m) & 0.5 \\
$\mathrm{y}$-domain $(\mathrm{m})$ & 0.1 \\
Fluid viscosity $(\mathrm{kg} / \mathrm{ms})$ & $10^{-18}$ \\
Time step (s) & 0.01 \\
Max residual for $\theta_{p}, \mathbf{u}_{g}$ and $\delta p$ & $10^{-9}$ \\
Relaxation on $\theta_{p}, \mathbf{u}_{g}$ and $\delta p$ & $1,1,1$ \\
Inlet gas velocity $(\mathrm{gas}$ only) $(\mathrm{m} / \mathrm{s})$ & 0.1 \\
Initial $\theta_{p}$ & 0.01 \\
\hline \hline
\end{tabular}


With a no-slip boundary, particle velocities will vary linearly between the first node free stream velocity and the wall velocity. With no diffusion, momentum transferred from the wall to particles near the wall will not propagate through particles across the channel. Figure 10 shows the movement of particles at $0.4 \mathrm{~s}$ time intervals. Particles between the first interior node and the wall follow the wall velocity. The particles retain their shape with no shear from diffusion, and the fluid velocity remains uniform across the channel as shown in Figure 11. The bottom and top fluid velocities increase and decrease, respectively, because of a change in fluid flow area down the channel (a decrease or increase in particle volume fraction).

The above example shows numerical diffusion not to be significant for the numerical formulation used in this work. As a second example, a $S^{T}$ interpolation function is used for the fluid velocity at a particle position in the interphase momentum transfer term in equation (40). Figure 12 shows the particle distributions at $0.4 \mathrm{~s}$ time intervals. Particles have significant shear as momentum is transferred through the particles, and the velocity approaches a nearly linear profile across the channel which is similar to diffusive Couette flow.

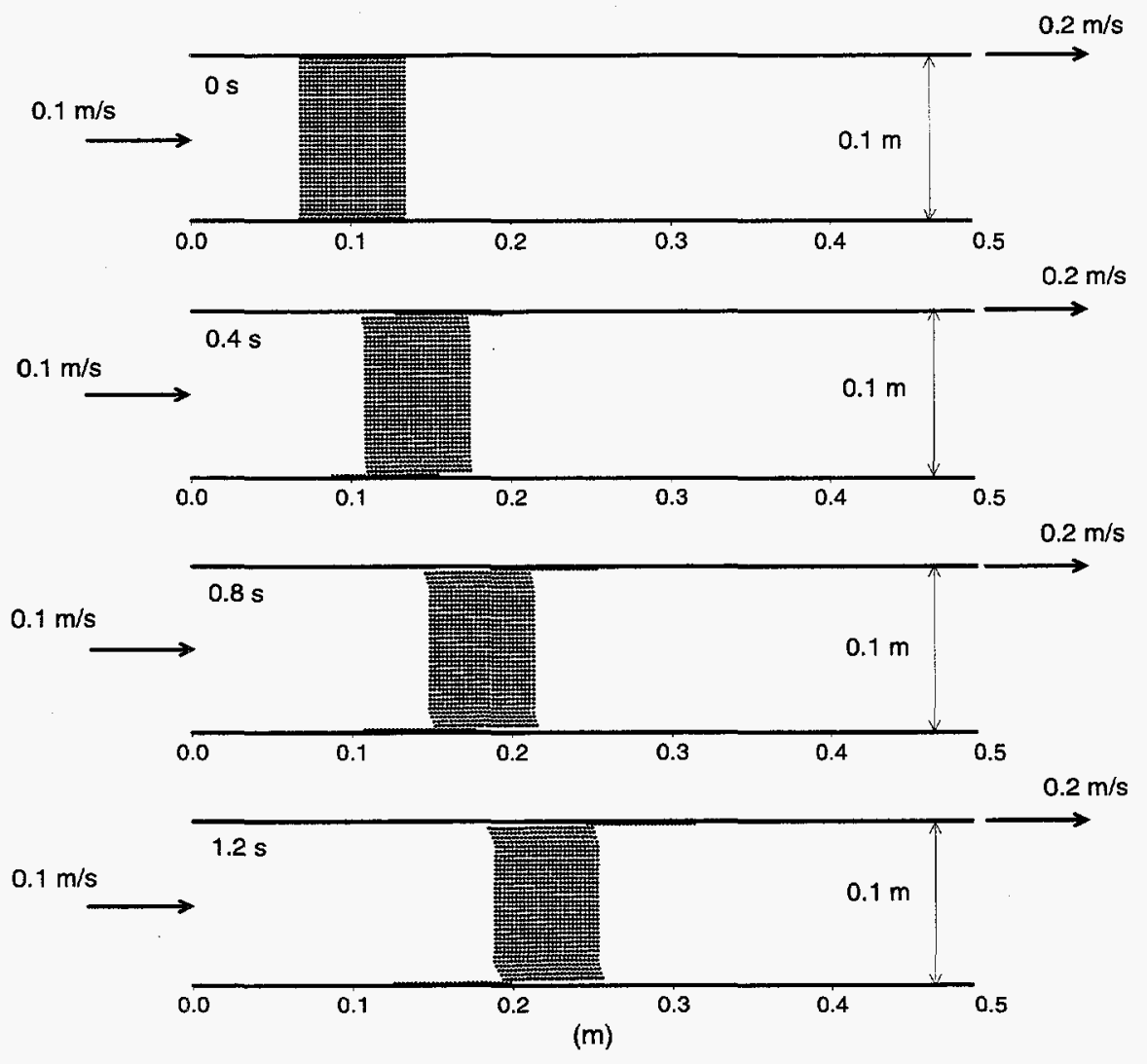

Figure 10. Particle flow between flat plates. Gas velocity at particle position from $S^{S}$. 


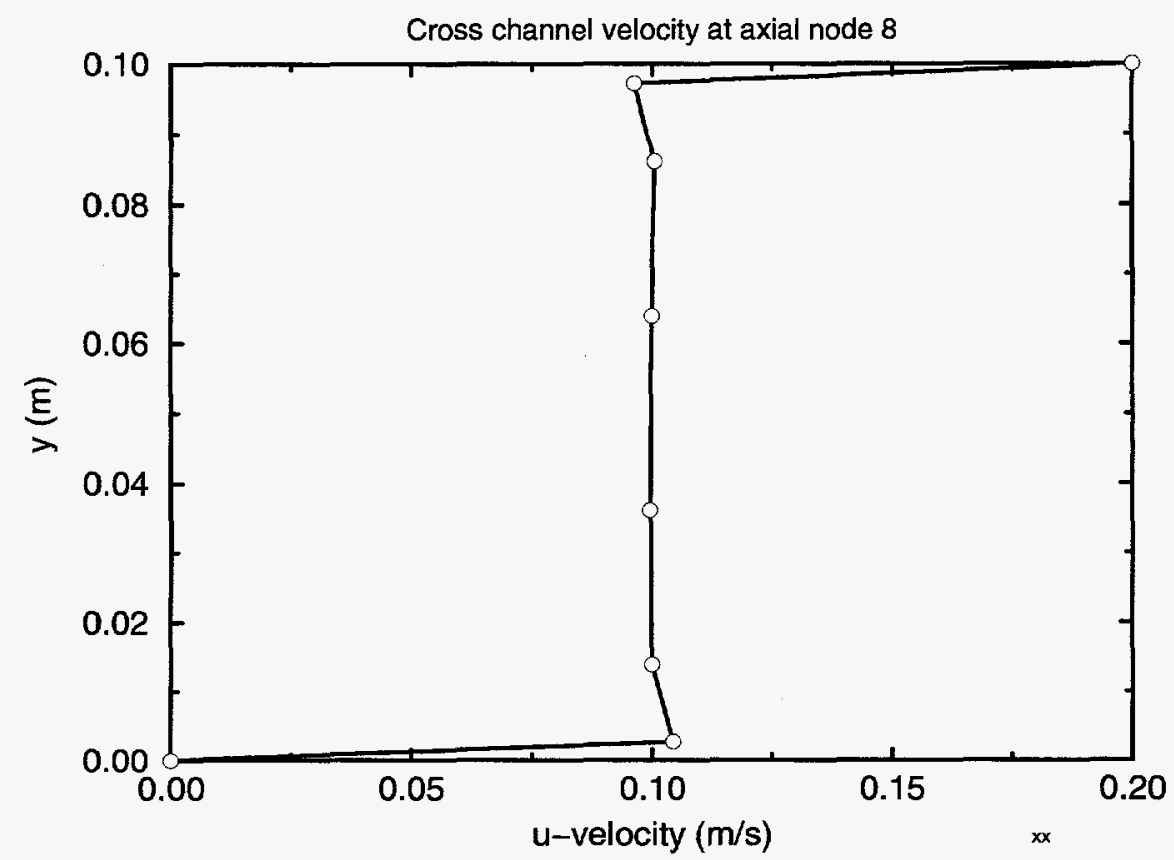

Figure 11. Fluid velocity profile for particle flow between flat plates. Gas velocity at particle position from $S^{S}$.
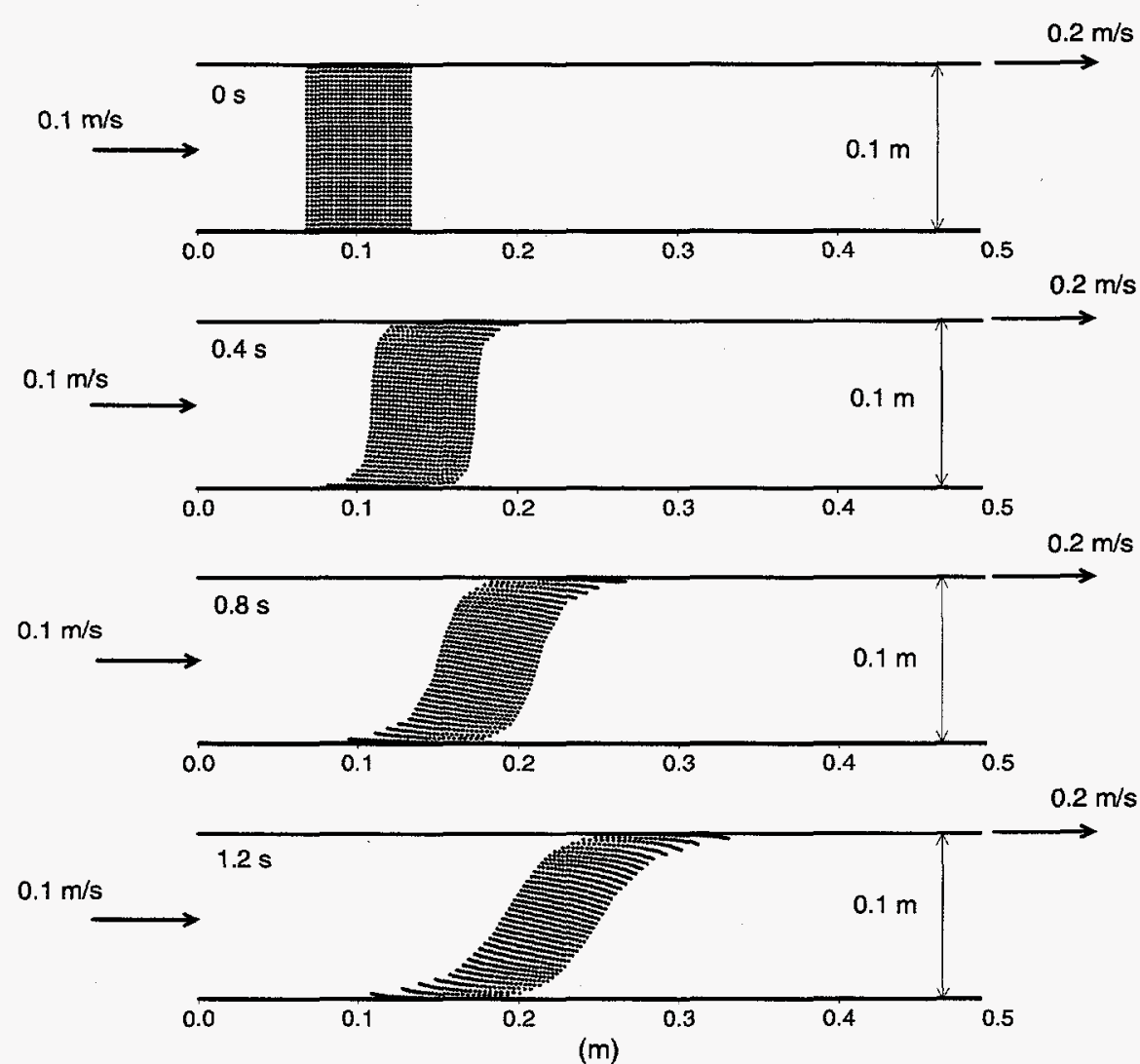

Figure 12. Particle flow between flat plates. Gas velocity at particle position from $S^{T}$. 


\section{BOUNDARY CONDITIONS}

\section{A. Interpolation function}

The interpolation from particles to the boundary face-center nodes is the same interpolation used for interior nodes. This gives a volume fraction at boundaries (face-center nodes) based on the particle distribution field. For cell center nodes, the interpolation function for particles between a boundary and the first interior node is unity. That is, the particle volume is mapped totally to the first interior cell-center node.

The gradient of the interpolation function, $\nabla S$, can be handled in two ways at boundaries. The gradient can be set to zero, or the gradient can be evaluated the same as interior nodes. In the implicit update of the particle volume fraction, the gradient must be zero to insure conservation of volume on the Eulerian grid. In calculating particle positions, if $\nabla S=0$ at the boundary, particles near the boundary are not driven by pressure or interparticle stress gradients, and any particle that passes the first interior node will stack on the boundary. Except for the Eulerian volume fraction equation, the interpolation function gradient is calculated the same for interior and boundary nodes.

\section{B. Particle properties}

Particles that intersect a rigid boundary are positioned a distance from the wall based on the particle volume fraction for the boundary node. The distance from a vertical wall is $d_{x}=\theta_{p} V_{o, j} / \Delta y_{j}$, where $\theta_{p}$ is the particle volume fraction at the boundary and $V_{o j}$ is the cell volume. The geometry is shown in Figure 13. Particles that cross a reflective or center-line boundary are reflected back into the calculation domain. The normal-to-wall component of the velocity is set to zero for particles impinging a rigid boundary and set negative for particles crossing a reflective or centerline boundary. The parallel-to-wall component of the particle velocity is the wall velocity for a noslip rigid boundary and is unchanged for a slip rigid boundary or centerline boundary. If a particle reflection leads to another boundary intersection, a rigid boundary condition is applied.

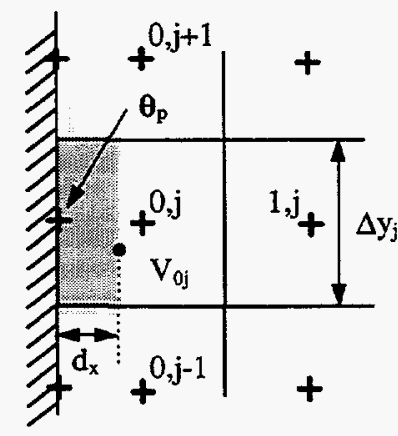

Figure 13. Positioning a particle which hits the left boundary. 


\section{Fluid properties}

The pressure at a solid boundary is taken to be the pressure at the nearest interior cell, and the pressure for an open boundary is specified. The fluid volume fraction is specified for an inlet flow and is $\theta_{g_{\text {wall }}}=1-\theta_{p_{\text {wall }}}$ for all other boundary types. At no-slip rigid walls, the normal-towall velocity is zero and the parallel-to-wall velocity is the wall velocity. For a slip boundary the normal-to-wall velocity is zero and the parallel-to-wall velocity is the velocity of the first interior node. Inlet flow boundaries have a specified velocity and volume fraction, and exit flow boundaries assume a one-way flow with fluid discharging to a fixed pressure sink.

\section{APPROXIMATION OF THE IMPLICIT PARTICLE VOLUME FRACTION}

By summing over all Eulerian nodes, it can be shown that the implicit particle volume fraction equation satisfies volume continuity over the calculation domain, and hence, the volume of particles remains constant. However, within individual cells, the Eulerian calculation of particle volume fraction may give volume fraction errors in satisfying volume continuity. Because an accurate Lagrangian calculation of particle volume fraction is made at the end of the time step, the error in Eulerian volume fraction is limited to the implicit iteration loop for fluid velocities and pressure.

Conservation of volume over the calculation domain is shown by summing the volume fraction equation (56) over all nodes, $\zeta$ which gives

$$
\sum_{\zeta} V_{\zeta} \theta_{p_{\zeta}}^{n+1}=\sum_{\zeta} V_{\zeta} \tilde{\theta}_{p_{\zeta}}^{n}
$$

where all other terms sum to zero. The right side is the intermediate-time volume fraction from summation of particle volumes over the calculation domain and thus conserves volume. In addition, the approximate particle volume fraction, on the left of (56), is also conserved. The summing to zero of other terms in equation (56) is next illustrated for the gravity term. Summing the gravity term over all nodes gives

$$
\begin{aligned}
& \sum_{\zeta} \Delta t^{2} \sum_{p} \frac{m_{p} N_{p}}{\rho_{p}\left(1+D_{p} \Delta t\right)} \nabla S_{\zeta}\left(\tilde{\mathbf{x}}_{p}\right) \cdot \mathbf{g} \\
= & \sum_{p} \frac{m_{p} N_{p} \Delta t^{2}}{\rho_{p}\left(1+D_{p} \Delta t\right)} \overbrace{\left\{\sum_{\zeta} \nabla S_{\zeta}\left(\tilde{\mathbf{x}}_{p}\right)\right\}}^{0} \cdot \mathbf{g}=0
\end{aligned}
$$

where the particle and volume summations have been interchanged. From the definition of $\nabla S$, the summation $\nabla S$ over all nodes is zero and conservation of volume is preserved.

The particle volume fraction equation (56) contains velocities, pressure and neighbor volume fractions, multiplied by explicit coefficients. The explicit coefficients result from summation of particle properties within four bounding Eulerian nodes as shown in Figure 2. This limits the implicit update of the particle volume fraction to the redistribution of volume within the four Eulerian nodes. In the Eulerian calculation, particles within the intermediate-step $S$ domain which 
are calculated to leave the domain at $t+\Delta t$ are forced to contribute to only the nodes supporting the intermediate-step $S$ domain. This can produce incorrect volume fraction within a cell and may lead to a non-physical negative volume fraction. An example is given below.
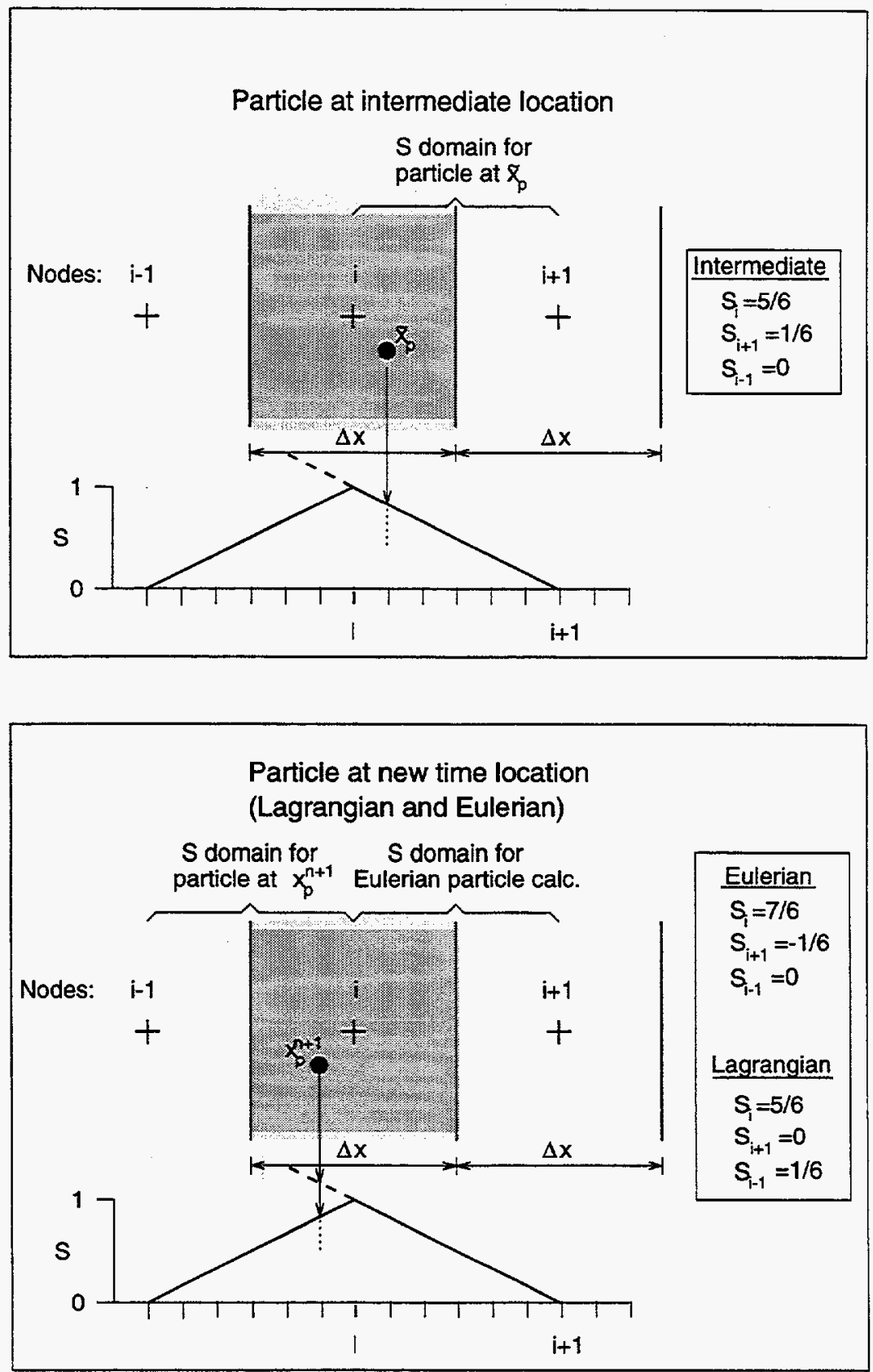

Figure 14. The implicit update of particle volume fraction.

Consider the one-dimensional case shown in Figure 14 where aerodynamic drag is the dominant force and the other terms are zero. A particle at the intermediate-step is located near 
node $i$, and within the $S$ domain defined within nodes $i$ and $i+1$. At the intermediate step, $5 / 6$ of the particle volume is mapped to node $i$ and $1 / 6$ is mapped to volume $i+1$. The implicit volume fraction at $t+\Delta t$ from equation (41) is

$$
\theta_{p_{\zeta}}^{n+1}=\tilde{\theta}_{p_{\zeta}}+\underbrace{\frac{\Delta t^{2}}{V_{\zeta}} \sum_{p} \frac{m_{p} N_{p}}{\rho_{p}\left(1+D_{p} \Delta t\right)} \nabla S_{\zeta}\left(\widetilde{\mathbf{x}}_{p}\right)}_{\text {explicit coefficient }} \cdot \tilde{\mathbf{u}}_{g, p}
$$

where the gas velocity, $\tilde{\mathbf{u}}_{g, p}=T_{\zeta{ }_{1 / 2}}\left(\mathbf{x}_{p}\right) \mathbf{u}_{g \zeta \mathrm{V} / 2}^{n+1}$, at particle position $\mathbf{x}_{p}$, is updated in the implicit iteration loop. The product $\nabla S_{\zeta}\left(\mathbf{x}_{p}\right) T_{\zeta 1 / 2}\left(\mathbf{x}_{p}\right)$ is not position dependent within the surrounding Eulerian nodes (Figure 14), and any distribution of particles within the domain of $S_{\zeta}$ will give the same explicit aerodynamic drag coefficient. For the one-dimensional example the particle volume fraction at bounding nodes $i$ and $i+1$ is

$$
\theta_{p_{i}}^{n+1}=\tilde{\theta}_{p_{i}}-\underbrace{\frac{\Delta t^{2}}{\Delta x} \sum_{p} \frac{V_{p}}{\left(1+D_{p} \Delta t\right)} \frac{1}{\delta x}\left[T_{i+1 / 2}\left(\tilde{x}_{p}\right)\right]^{2}}_{\text {explicit coefficient } c_{e}} \tilde{u}_{g_{i+1 / 2}}
$$

and

$$
\theta_{p_{i+1}}^{n+1}=\tilde{\theta}_{p_{i+1}}+\underbrace{\frac{\Delta t^{2}}{\Delta x} \sum_{p} \frac{V_{p}}{\left(1+D_{p} \Delta t\right)} \frac{1}{\delta x}\left[T_{i+1 / 2}\left(\tilde{x}_{p}\right)\right]^{2}}_{\text {explicit coefficient } c_{e}} \tilde{u}_{g_{i+1 / 2}}
$$

where $V_{p}$ is a particle's volume. The volume increases or decreases at Eulerian node $i$, as the gas velocity moves to the left or the right, respectively. What is lost by node $i$ is gained by node $i+1$ and conservation of volume is preserved:

$$
\begin{gathered}
\theta_{p_{i}}^{n+1}=\tilde{\theta}_{p_{i}}-c_{e} \tilde{u}_{g_{i+1 / 2}} \\
\theta_{p_{i+1}}^{n+1}=\tilde{\theta}_{p_{i+1}}+c_{e} \tilde{u}_{g_{i+1 / 2}} .
\end{gathered}
$$

Figure 14 shows the particle moves to the left outside the support of $S_{i}\left(\tilde{x}_{p}\right)$ at $t+\Delta t$. This is true in both the Eulerian calculation and the Lagrangian calculation at $t+\Delta t$. The Eulerian implicit calculation continues to use the $S$ function defined for the particle at the intermediate step. The intermediate $S$ function gives 7/6 volume to node $i,-1 / 6$ volume to node $i+1$, and none to node $i$ 1. The Lagrangian new $S$ function for the particle gives the correct $5 / 6$ volume to node $i$, zero volume to node $i+1$, and $1 / 6$ volume to node $i-1$. The result is an inaccurate Eulerian calculation of volume fraction and a negative volume fraction which is non-physical. The error is more pronounced at low numbers of particles and very dynamic conditions. The error in volume redistribution may effect the fluid velocity calculation, primarily through the rate of change of 
volume fraction in the pressure correction, and it can lead to higher interparticle stresses. Note that if the particle in the example stays within its intermediate-step $S$ domain, the implicit volume fraction is accurate.

The volume error arises only in the Eulerian calculation of particle volume fraction step which is used in the implicit calculation loop for continuum velocities and pressure. At the end of the calculation step, the true volume fraction is calculated from a Lagrangian update of particle positions. The error in Eulerian calculated volume fraction is usually small and is reduced by increasing the number of particles. To eliminate non-physical negative volume fraction in the implicit loop, the volume fraction is limited to 0 . To reduce high interparticle stress, during the implicit iteration, the volume fraction is limited to $99 \%$ of the close-pack volume fraction.

\section{SOLUTION PROCEDURE}

The solution begins with an intermediate Lagrangian calculation of the particle positions and particle volume fraction based on the old particle velocities. New-time fluid velocity and pressure are calculated in an implicit inner iteration loop. The inner loop uses the Eulerian calculation of the particle volume fraction. The sets of Eulerian algebraic equations are solved using a conjugate gradient solver (O'Rourke and Amsden 1986). The implicit iteration loop continues until no iterations are required in the conjugate gradient pressure solver, where convergence is satisfied when the pressure correction residuals at all nodes are less than a specified limit (typically $10^{-6}$ to $10^{-10} \mathrm{~Pa}$ ). At the completion of the time step, a Lagrangian calculation of new particle velocities, positions, and volume fraction is made. The solution steps are outlined in Figure 15.

\section{INTERPARTICLE STRESS}

The interparticle stress term given by equation (11),

$$
\tau=\frac{P_{s} \theta_{p_{p}}^{\beta}}{\theta_{c p}-\theta_{p}},
$$

is an empirical equation to account for particle collisions as particles congregate. It has the desired characteristic of repelling particles from an Eulerian volume if the density of particles approaches the close-pack limit. The close-pack volume fraction, $\theta_{c p}$, can be defined for spheres with simple arrangements. However, $\theta_{c p}$ is generally measured for a bed and typically ranges from 0.6 to 0.7 . In calculating a static state, the $P_{s}$ term has minor influence. The gradient supporting the weight of a particle bed is derived principally by the small volume fraction difference in the denominator in equation (11). However $P_{s}$ is important in dynamic calculations. The $P_{s}$ must be sufficient to stop the momentum of particles encroaching into a nearly close-packed volume such that the particles do not compress together beyond their close-pack limit. However, a large value of $P_{s}$ disperses particles even at low volume fractions where particle collisions are not large. The power, $\beta$, on volume fraction in the interparticle stress equation reduces the particle dispersion at low volume fraction. The $P_{s}$ and $\beta$ parameters are empirical for which little measured data are available. The value used for $\beta$ in this study ranged from 1 to 3 , with the typical value being 2 . The value for $P_{s}$ ranged from $5 \mathrm{~Pa}$ to $200 \mathrm{~Pa}$ with the typical value being $100 \mathrm{~Pa}$. 


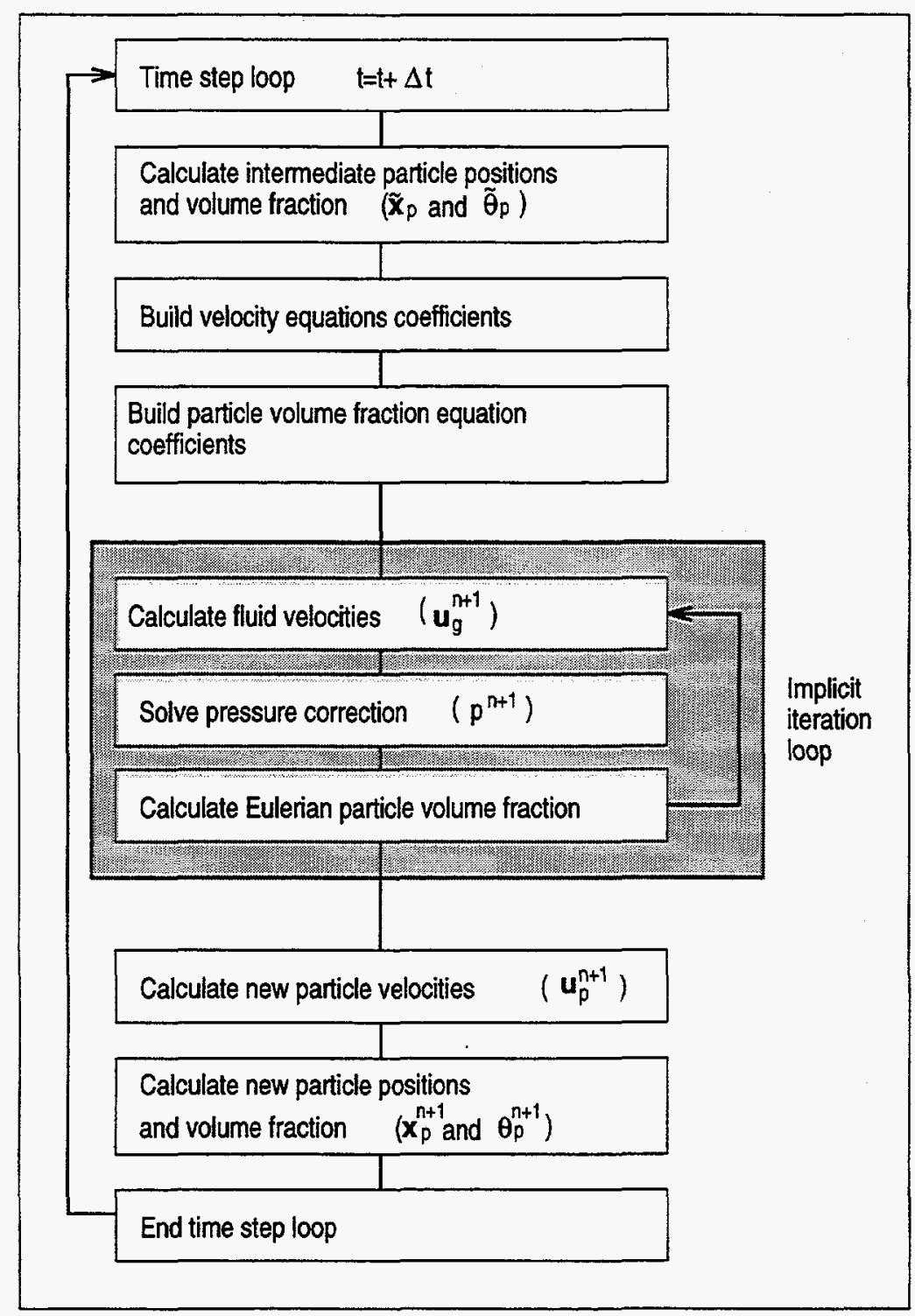

Figure 15. Diagram of calculation scheme with implicit particle volume fraction. 


\section{ONE-DIMENSIONAL TEST PROBLEMS}

In this section, the two-dimensional MP-PIC method is compared with one-dimensional problems that have known analytical solutions. One-dimensional problems are run in two ways using the two-dimensional code. First, problems have been solved in both the $\mathrm{x}$ and $\mathrm{y}$ direction to verify consistency in the solution. Second, two-dimensional problems are run with no initial perturbations which gives a one-dimensional behavior. The computed results are compared with the analytical solutions.

\section{A. Stress wave problem}

Gidaspow (1986) gave an analytical solution for the propagation speed of a small density fluctuation for a dense particle flow in a vacuum. The interparticle stress is given by equation (11) where $\beta=1$. Andrews and O'Rourke (1995) used the stress wave problem to validate the onedimensional MP-PIC method. The aerodynamic and gravitational forces are zero. A small initial step in particle volume fraction of 0.004 is applied. The fluid velocity is small and pressure gradients are negligible. The particle stress wave speed is (Gidaspow 1986)

$$
c_{s}=\sqrt{\frac{G\left(\theta_{0}\right)}{\rho_{s}}} \text { where } G\left(\theta_{0}\right)=\frac{P_{s} \theta_{c p}}{\left(\theta_{c p}-\theta_{0}\right)^{2}},
$$

where $\beta=1$. Specifications of the test problem are given in Table 2 . Figure 16 shows the onedimensional stress wave propagation. Two opposite moving waves occur with the analytic wave speed of $0.1183 \mathrm{~m} / \mathrm{s}$. Measuring the distance between the numerical expanding waves at time intervals gives a wave speed which matches that from the analytic solution. From Figure 16, the calculated wave speed is $0.116 \mathrm{~m} / \mathrm{s}$.

\begin{tabular}{lc}
\hline Table 2. Stress wave problem with a single $\mathrm{x}$-channel. \\
\hline & \\
Number of parcels & 2000 \\
Particle radius $(\mathrm{m})$ & 0.001 \\
Particle density $\left(\mathrm{kg} / \mathrm{m}^{3}\right)$ & 1000 \\
Fluid density $\left(\mathrm{kg} / \mathrm{m}^{3}\right)$ & 1 \\
$P_{\mathrm{s}}(\mathrm{Pa})$ & 5 \\
$\theta_{\mathrm{cp}}$ & 0.7 \\
$\mathrm{x}$-gravity, y-gravity $\left(\mathrm{m} / \mathrm{s}^{2}\right)$ & 0,0 \\
Number $\mathrm{x}$-cells & 1 \\
Number y-cells & 50 \\
x-domain $(\mathrm{m})$ & 0.01 \\
y-domain $(\mathrm{m})$ & 2 \\
Time step $(\mathrm{s})$ & 0.005 \\
Max residual for $\theta_{\mathrm{p}}, \mathrm{u}_{\mathrm{g}}$ and $\delta \mathrm{p}$ & $10^{-8}$ \\
\hline \hline
\end{tabular}



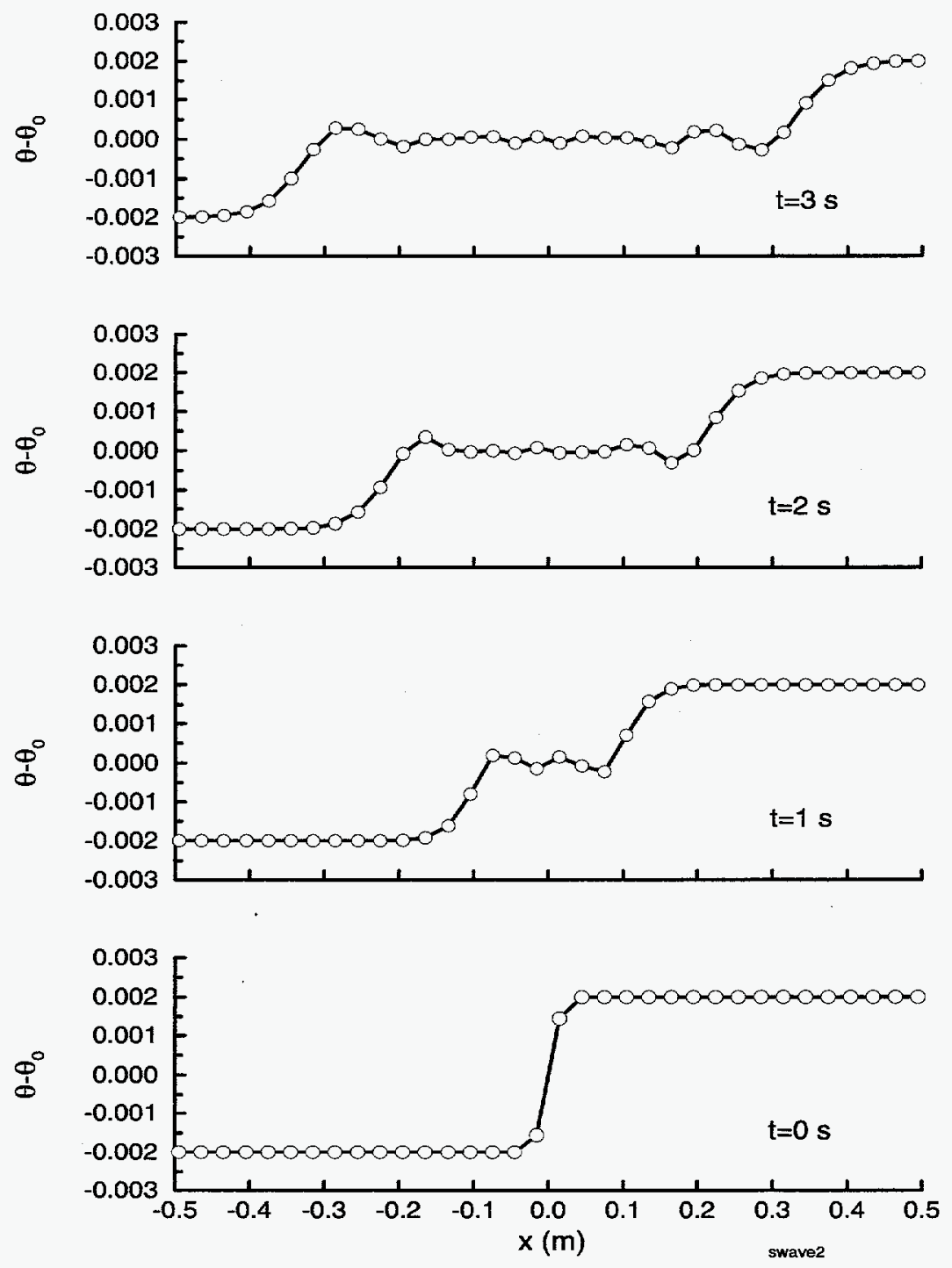

Figure 16. Particle volume fraction for a stress wave. 


\section{B. One-dimensional layered sedimentation}

The one-dimensional sedimentation of a particle-fluid mixture above a lighter fluid is calculated (Andrews and O'Rourke 1995). The problem considers gravity driven particles falling through gas and depositing at the bottom of the container as illustrated in Figure 17. The test problem conditions are given in Table 3 .

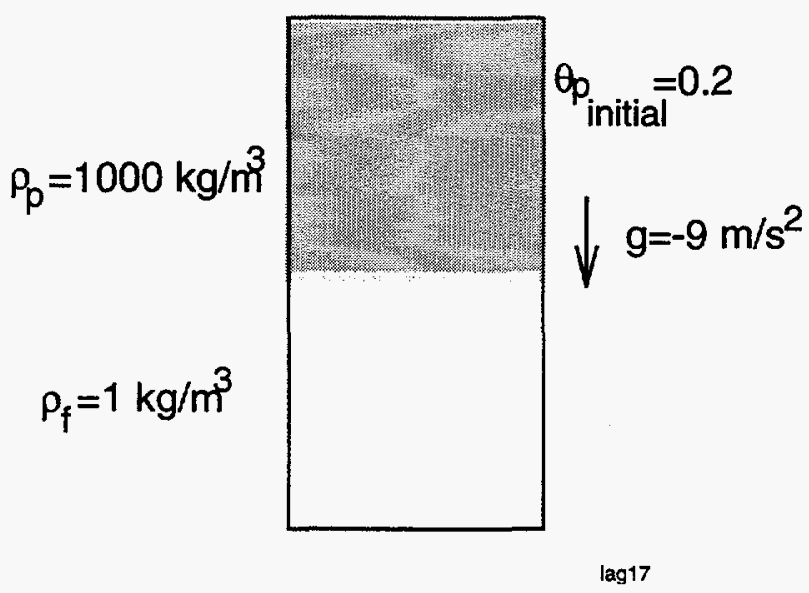

Figure 17. Illustration of layered sedimentation problem.

The initial sedimentation of particles before stacking onto the bottom has an analytic solution (Andrews and O'Rourke 1996),

$$
\theta= \begin{cases}v^{-1}(x / t) & v\left(\theta_{-\infty}\right) t \leq x \leq v\left(\theta_{+\infty}\right) t \\ \theta_{-\infty} & x<v\left(\theta_{-\infty}\right) t \\ \theta_{+\infty} & x>v\left(\theta_{+\infty}\right) t\end{cases}
$$

where $v=\frac{g\left(\theta_{g}\right)^{k}}{D_{p}}\left[(2+k) \theta_{p}-1\right]$ with $k=3.65$ and $\rho_{p}>>\rho_{g}$.

The particle volume fraction profile at various times is shown in Figure 18. Particles at position $\boldsymbol{C}$ fall at near the free-fall velocity $g / D_{p}=0.1 \mathrm{~m} / \mathrm{s}$ and reach the bottom in $5 \mathrm{~s}$. At point $\boldsymbol{B}$, the particle volume fraction is 0.2 and the mixture edge rises at $0.00576 \mathrm{~m} / \mathrm{s}$. The top edge of the mixture, $\boldsymbol{A}$, falls at the solids velocity $\left(\theta_{g}\right)_{0}\left(\theta_{g}\right)^{k} \frac{g}{D_{p}}$, where $\left(\theta_{g}\right)_{0}=0.8$. Figure 18 shows the numerical calculation compares well with the analytic solution. Figure 18 also shows the settling of particles at $10 \mathrm{~s}, 15 \mathrm{~s}, 25 \mathrm{~s}$ and $40 \mathrm{~s}$. At $25 \mathrm{~s}$ most particles have settled to the bottom with a volume fraction near close-packing. Both the two-dimensional calculation with no initial perturbations and the calculations with a single channel compare well with the analytic solution and with results given by Andrews and O'Rourke (1996). 

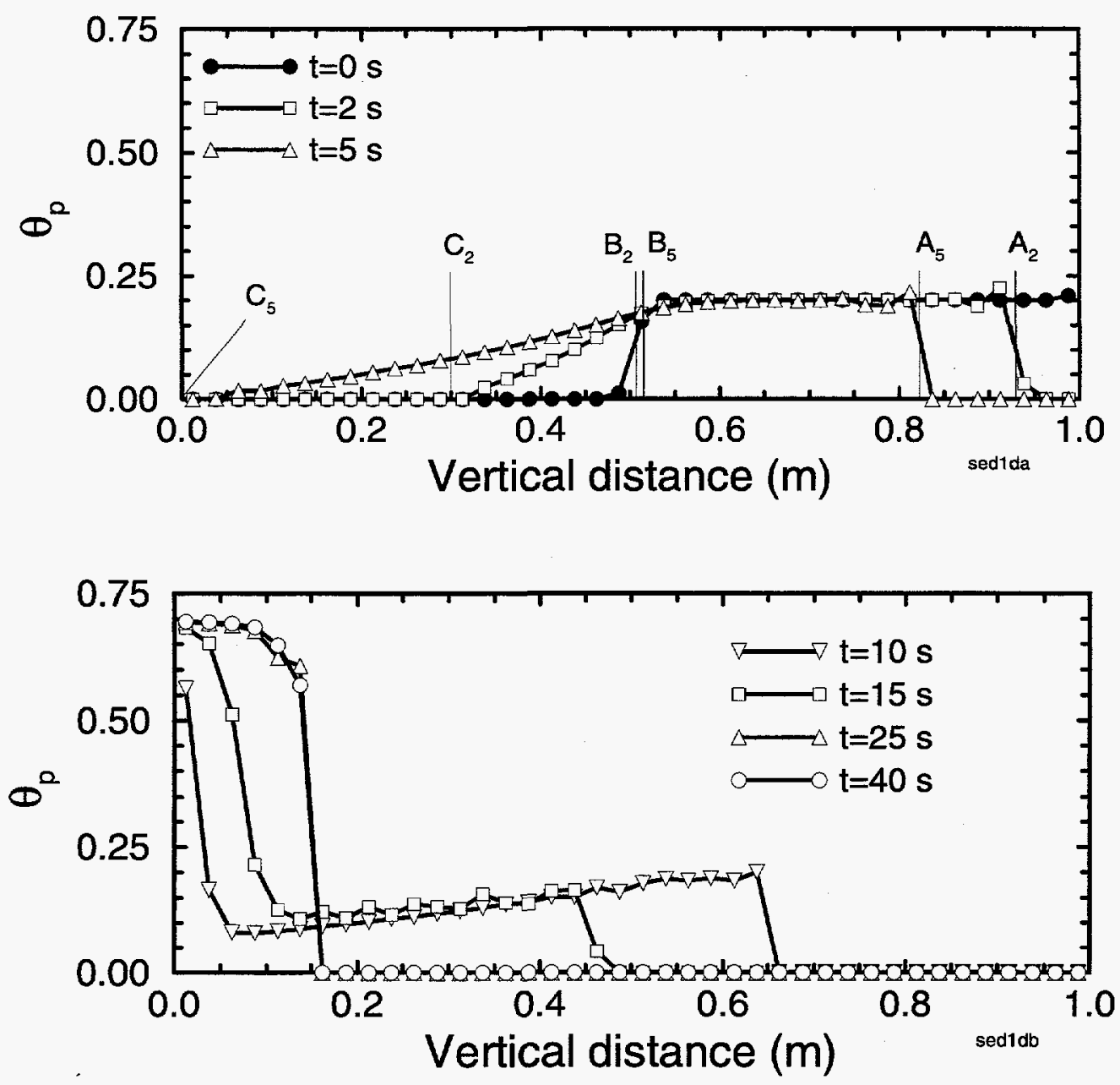

Figure 18. One-dimensional sedimentation. 
Table 3. One-dimensional sedimentation.

\begin{tabular}{lc} 
Number of parcels & 200 \\
Number particles in a parcel & 59,683 \\
Particle radius $(\mathrm{m})$ & 0.001 \\
Particle density $\left(\mathrm{kg} / \mathrm{m}^{3}\right)$ & 1000 \\
Fluid density $\left(\mathrm{kg} / \mathrm{m}^{3}\right)$ & 1 \\
$P_{s}(\mathrm{~Pa})$ & 5 \\
$\theta_{c p}$ & 0.7 \\
x-gravity, y-gravity $\left(\mathrm{m} / \mathrm{s}^{2}\right)$ & $0,-9$ \\
Number x-cells & 1 \\
Number y-cells & 40 \\
x-domain $(\mathrm{m})$ & 1 \\
y-domain $(\mathrm{m})$ & 1 \\
Fluid viscosity $(\mathrm{kg} / \mathrm{ms})$ & 0.02 \\
Time step $(\mathrm{s})$ & 0.002 \\
Max residual for $\theta_{p}, \mathbf{u}_{g}$ and $\delta p$ & $10^{-7}$ \\
\hline \hline
\end{tabular}

\section{Solid stress wave speed limit on time step}

The solid stress wave speed limits an explicit calculation time step. A stress speed courant number is defined as $\frac{c_{s} \Delta t}{\Delta x}$, where $c_{s}$ is given by equation (70). A stress speed courant number greater than unity leads to numerical instabilities in the explicit solution. Figure 19 shows that, for the MP-PIC implicit solution, the stress speed courant number for the one-dimensional sedimentation problem well exceeds the explicit limit of unity. Computation details for calculations shown in Figure 19 are listed in Table 3 with the exception of calculation time steps which are $0.02 \mathrm{~s}$ and $0.05 \mathrm{~s}$.

\section{TWO-DIMENSIONAL HEAVY PARTICLE MIXTURE ABOVE A LIGHT FLUID}

Two dimensional calculations for heavy particle mixtures above a light fluid are presented. The first calculation is a single wave-length Rayleigh-Taylor problem. The interface drag is large, tightly locking particles and fluid together. Results are compared with linear stability analysis and with experimental limiting growth rate data. The second calculation is a repeat of the previous one-dimensional sedimentation calculation. An initial interface perturbation is applied which gives rise to Rayleigh-Taylor type instabilities. The third set of problems comprises two-dimensional Rayleigh-Taylor mixing where instability generated structures combine to form ever larger structures. The calculated interpenetration of heavy particle mixture and lighter fluid is compared with experimental data. 


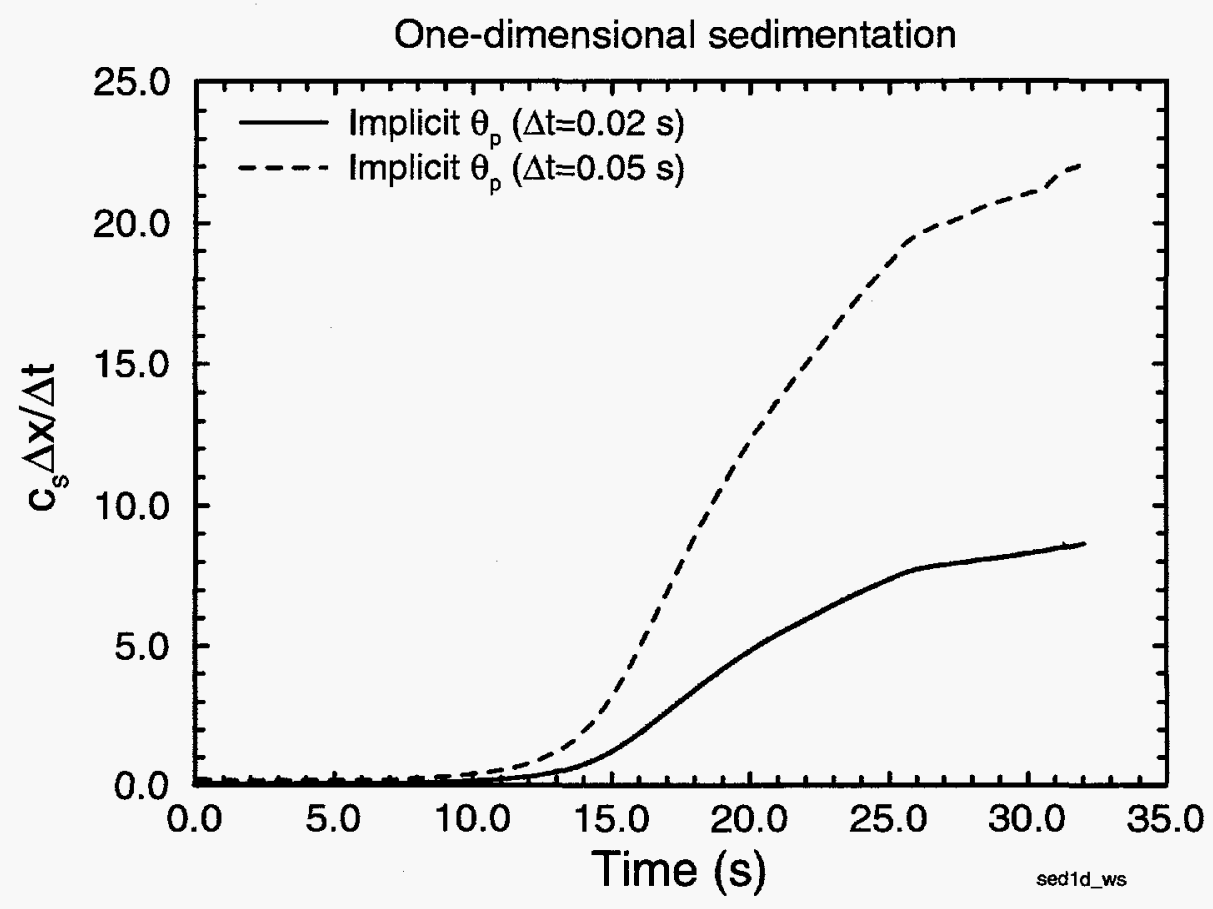

Figure 19. Stress wave courant number for the 1-D sedimentation problem.

\section{A. Rayleigh-Taylor single wave instability}

A density difference with heavy fluid above light fluid under gravity drives small amplitude Rayleigh-Taylor instabilities at the density interface to grow exponentially (Taylor 1950 and Chandrasekhar 1961). Linear instability analysis gives an exponential growth rate of a single structure in the absence of surface tension and viscosity with the amplitude given by

$$
h=A_{o} \cosh \left(n_{\lambda} t\right)
$$

where

$$
n_{\lambda}=\sqrt{\frac{2 \pi g}{\lambda} \frac{\rho_{2}-\rho_{1}}{\rho_{2}+\rho_{1}}},
$$

and $t$ is time, $g$ is gravity, $\lambda$ is the perturbation wavelength, and $\rho_{1}$ and $\rho_{2}$ are the densities of the lower and upper layers, respectively. $A_{o}$ is the initial perturbation amplitude.

Experimental and numerical work by Lewis (1950), Birkoff (1960), and Daly (1967) suggests that when the amplitude of a growing single Rayleigh-Taylor structure becomes significant compared to its wavelength, the growth rate slows and approaches a limiting value given by

$$
V_{\infty}=C_{\infty} \sqrt{g \frac{\lambda}{4} \frac{\rho_{2}-\rho_{1}}{\rho_{2}}},
$$


where the coefficient $C_{\infty}$, has been measured by Ratafai (1973) and Daly (1967) and found to be near 0.7 . This analysis uses $C_{\infty}=0.7$ for comparison with the MP-PIC calculation.

A MP-PIC calculation of a single Rayleigh-Taylor plume is made. The initial condition is a mixture of heavy particles and fluid situated above pure fluid. Initially a half sine wave perturbation in volume fraction is given to the interface between the mixture and pure fluid. The interphase drag is set high at $10^{8} \mathrm{~s}^{-1}$ to lock fluid and particles together, and the upper layer particle volume fraction is 0.1 . The fluid density is $1 \mathrm{~kg} / \mathrm{m}^{3}$ and calculations for particle densities $2,3,4$, and $6 \mathrm{~kg} / \mathrm{m}^{3}$ are made. Because the upper layer is a mixture, the effective light to heavy density ratios for the cases are 1.1,1.2,1.3, and 1.5. The problem conditions are given in Table 4 .

Figure 20 shows the transient Rayleigh-Taylor plume for a density ratio of 1.2. The plumes have the distinctive Rayleigh-Taylor mushroom shape. Figure 21 compares the calculated plume growth rates for the 4 density ratios with the rate predicted by linear stability analysis and the rate for saturated growth. The plume penetration is normalized with respect to the initial applied wavelength, $\lambda_{o}$. The time is normalized by the limiting growth time $\left[\lambda_{o} / g A\right]^{1 / 2}$, where the Atwood number is $A=\left(\rho_{2}-\rho_{1}\right) /\left(\rho_{2}+\rho_{1}\right)$. The calculation compares well with the experimental data.

A common method of normalizing Rayleigh-Taylor growth rate data is to initially shift the growth rate curve so that a straight line through the growth curve would pass through the origin (Linden, et al., 1994). This eliminates the influence of initial perturbations in reaching limiting growth rate. Figure 22 shows the calculated data after shifting the data to their zero time origin, normalizing the data, and then shifting data back to their original initial time value. The curves further collapse to a single curve. Again the calculation compares well with the saturated growth rate at constant velocity. 


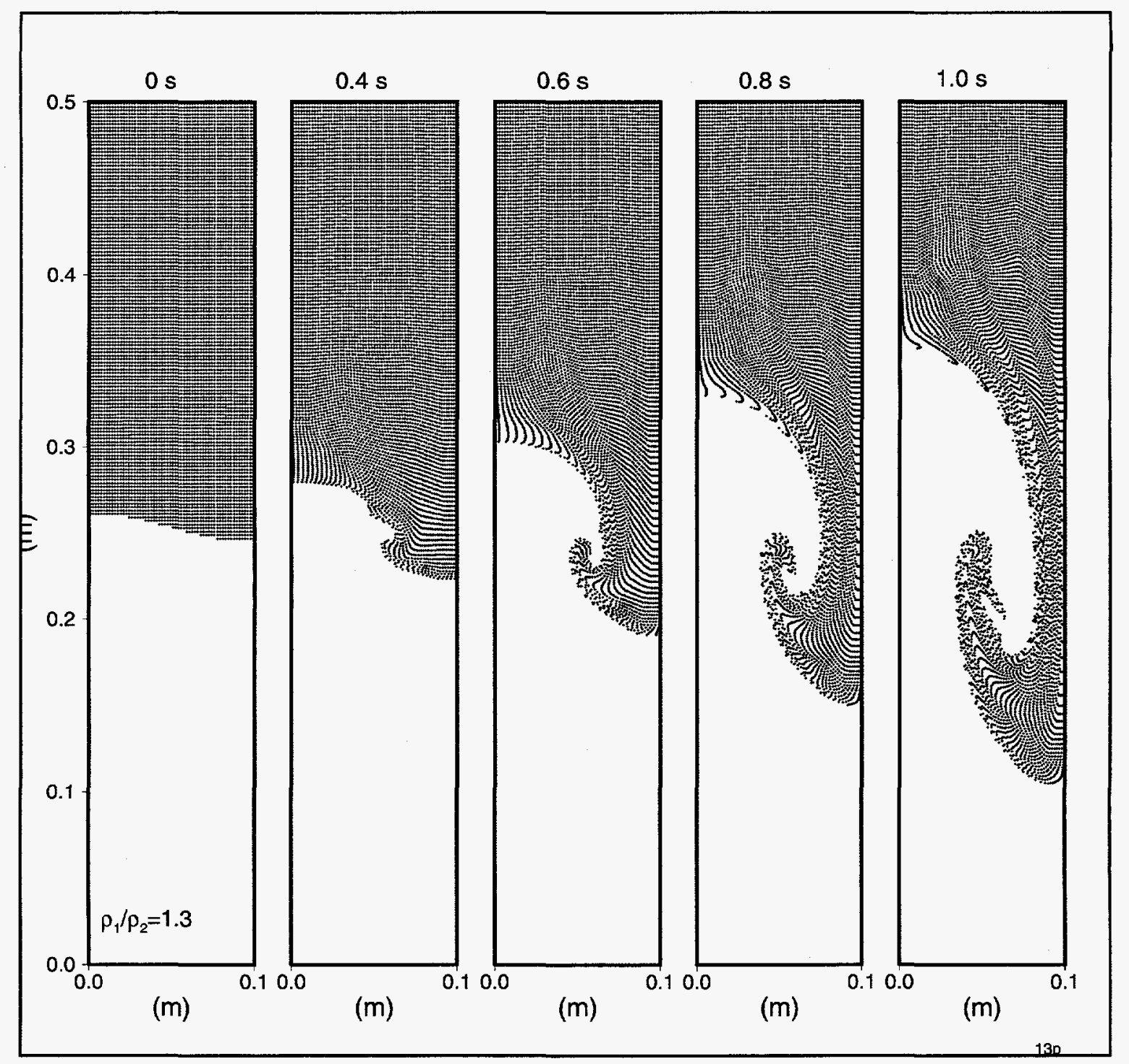

Figure 20. Rayleigh-Taylor single plumes. 


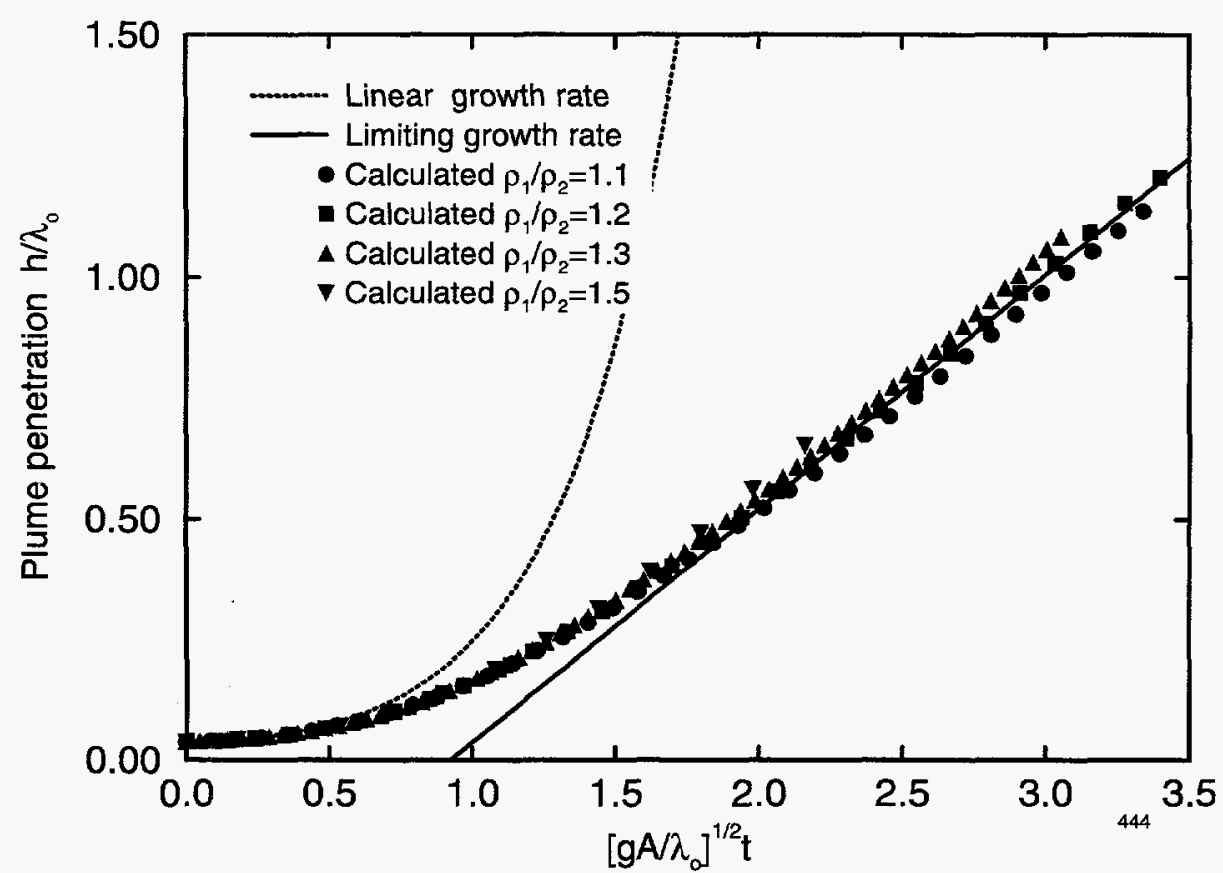

Figure 21. Comparison of calculated Rayleigh-Taylor plume growth rate with linear stability growth rate and measured saturated growth rate $\left(\mathrm{C}_{\infty}=0.7\right)$.

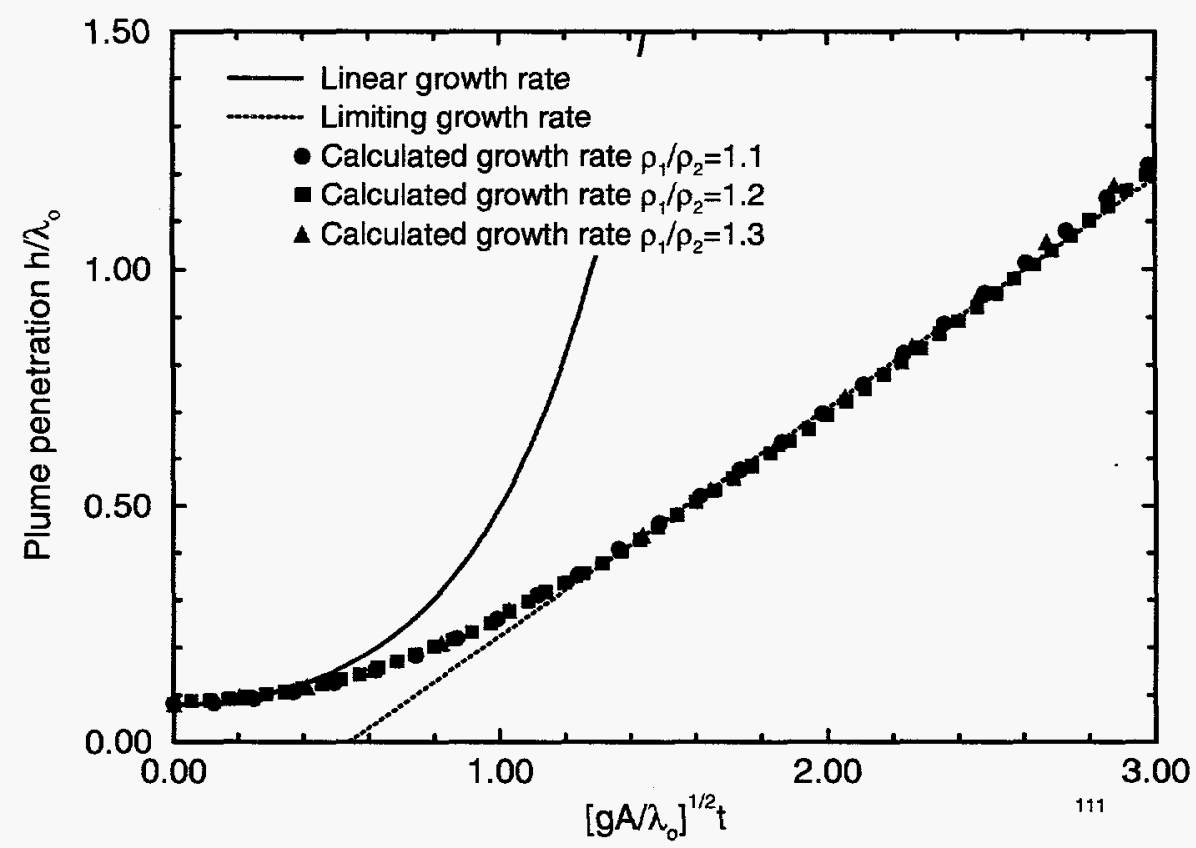

Figure 22. Comparison of calculated Rayleigh-Taylor plume growth rate with linear stability growth rate and measured saturated growth rate $\left(\mathrm{C}_{\infty}=0.7\right)$. Calculated curves are adjusted to a zero virtual origin. 


\begin{tabular}{lcccc}
\hline \hline \multicolumn{5}{c}{ Table 4. Rayleigh-Taylor problem. } \\
\hline & & & & \\
Particle volume fraction & 0.1 & 0.1 & 0.1 & 0.1 \\
Number particles & 8000 & 8000 & 8000 & 8000 \\
Particle radius (m) & 0.001 & 0.001 & 0.001 & 0.001 \\
Particle density $\left(\mathrm{kg} / \mathrm{m}^{3}\right)$ & 2 & 3 & 4 & 6 \\
Fluid density $\left(\mathrm{kg} / \mathrm{m}^{3}\right)$ & 1 & 1 & 1 & 1 \\
$P_{s}(\mathrm{~Pa})$ & 0 & 0 & 0 & 0 \\
X-gravity, y-gravity $\left(\mathrm{m} / \mathrm{s}^{2}\right)$ & $0,-9$ & $0,-9$ & $0,-9$ & $0,-9$. \\
Number x-cells & 5 & 5 & 5 & 5 \\
Number y-cells & 50 & 50 & 50 & 50 \\
X-domain (m) & 0.1 & 0.1 & 0.1 & 0.1 \\
y-domain (m) & 0.5 & 0.5 & 0.5 & 0.5 \\
Time step (s) & 0.002 & 0.002 & 0.002 & 0.002 \\
$A_{o}(\mathrm{~m})$ & 0.008 & 0.008 & 0.008 & 0.008 \\
Max residual for $\theta_{p}, \mathbf{u}_{g}$ and $\delta p$ & $10^{-7}$ & $10^{-7}$ & $10^{-7}$ & $10^{-7}$ \\
\hline \hline
\end{tabular}

The single, half-wave initial perturbation Rayleigh-Taylor problem is repeated at higher grid resolution. Figure 23 shows the calculated particle distribution at various times. Details of the calculation are given in Table 5. With the high grid resolution and discrete particles, the problem begins with small volume fraction variations along the applied half sine wave interface. Small perturbations grow quickly (from linear perturbation theory, small disturbances grow exponentially) and can be seen at the interface at $0.3 \mathrm{~s}$. Larger multiple wave length plumes are seen at $0.6 \mathrm{~s}$, and by $1 \mathrm{~s}$, plumes have paired forming a large single wavelength plume rotating in the cell. Figure 24 shows the problem calculated again with more than double the particles. The specifications are given in Table 5. With more particles the initial interface volume fraction is smoother and fewer small wavelength structures form. The calculated large structures for both grid densities at $0.6 \mathrm{~s}$ have nearly the same shape and interpenetration of mixture and fluid. 

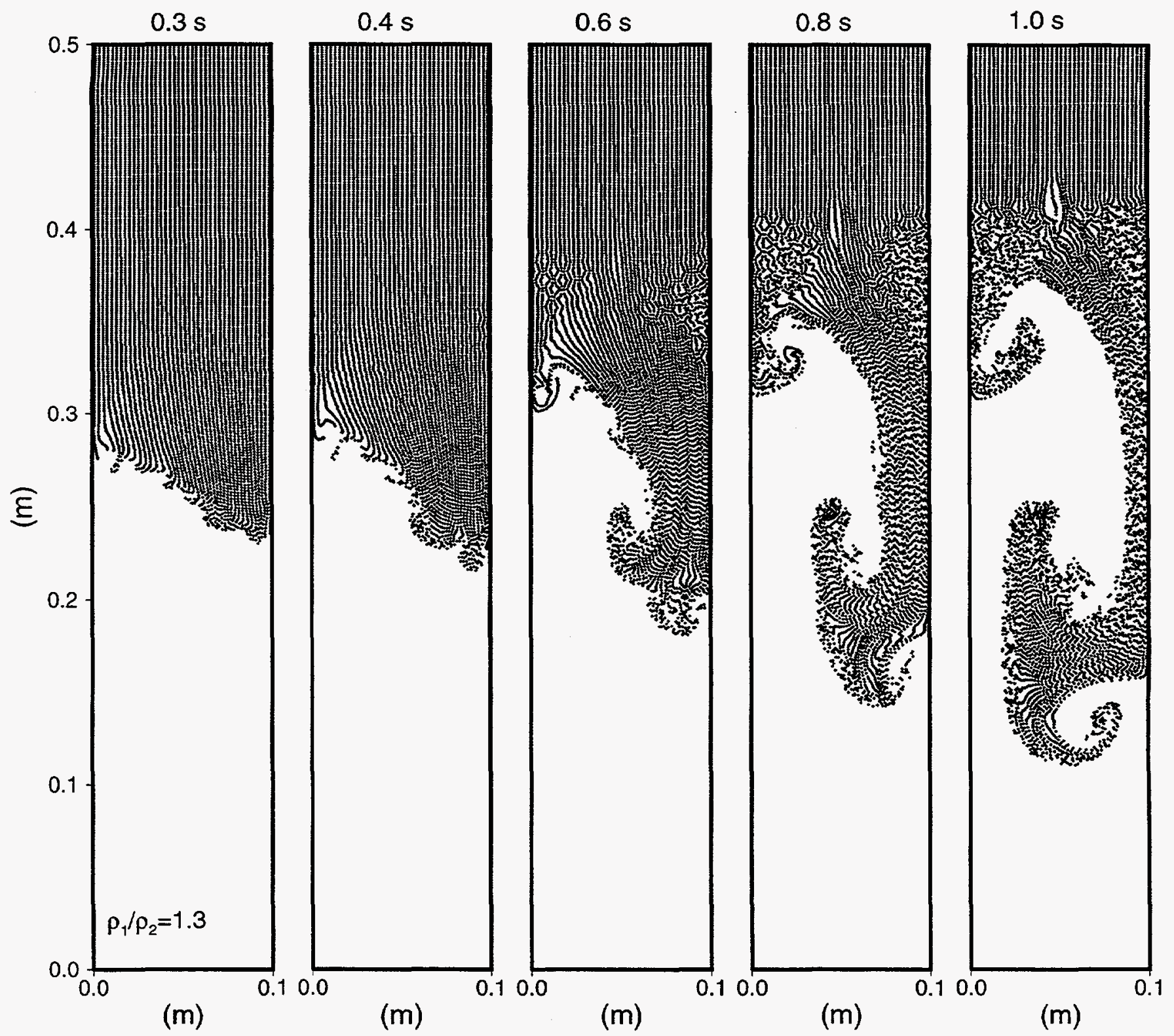

Figure 23. Rayleigh-Taylor single wave perturbation with grid resolution of $20 \times 100$. 


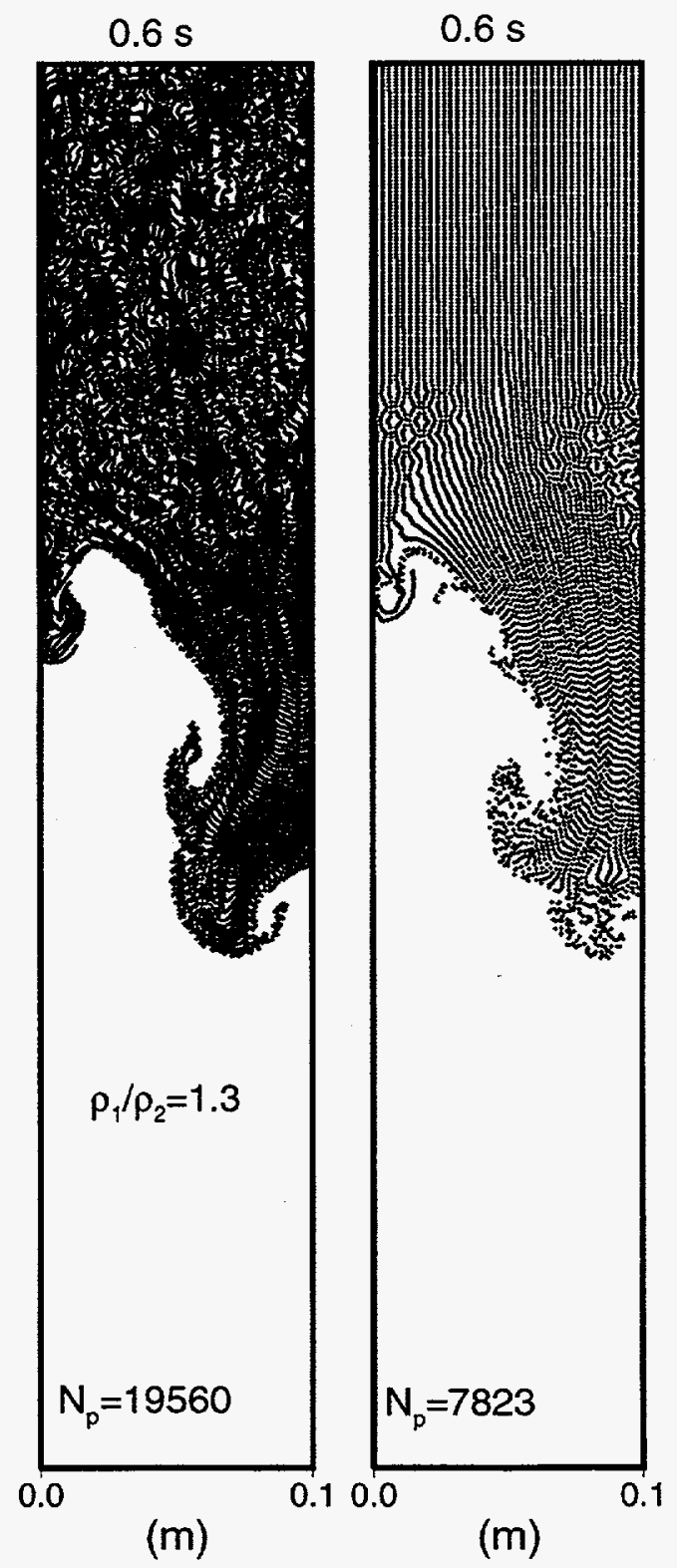

Figure 24. Rayleigh-Taylor single wave perturbation with grid resolution of $20 \times 100$ and 7,823 and 19,560 particles 
Table 5. Rayleigh-Taylor problem with nodalization of $20 \times 100$ and 7,823 and 19,560 particles.

\begin{tabular}{lcc} 
Particle volume fraction & 0.1 & 0.1 \\
Number particles & 7823 & 19560 \\
Particle radius $(\mathrm{m})$ & 0.001 & 0.001 \\
Particle density $\left(\mathrm{kg} / \mathrm{m}^{3}\right)$ & 4 & 4 \\
Fluid density $\left(\mathrm{kg} / \mathrm{m}^{3}\right)$ & 1 & 1 \\
$P_{s}(\mathrm{~Pa})$ & 0 & 0 \\
$\theta_{c p}$ & 0.7 & 0.7 \\
x-gravity, y-gravity $\left(\mathrm{m} / \mathrm{s}^{2}\right)$ & $0,-9$ & $0,-9$ \\
Number x-cells & 20 & 20 \\
Number y-cells & 100 & 100 \\
X-domain $(\mathrm{m})$ & 0.1 & 0.1 \\
y-domain $(\mathrm{m})$ & 0.5 & 0.5 \\
Time step $(\mathrm{s})$ & 0.002 & 0.002 \\
$A_{o}(\mathrm{~m})$ & 0.008 & 0.008 \\
Max residual for $\theta_{p}, \mathbf{u}_{g}$ and $\delta p$ & $10^{-7}$ & $10^{-7}$ \\
\hline \hline
\end{tabular}

\section{B. Two-dimensional layered sedimentation}

The one-dimensional sedimentation problem shown in Figure 18 is calculated again in two dimensions. In the two-dimensional case, instabilities are expected to form at the mixture-to-fluid interface if there are perturbations in the volume fraction field. Without perturbations, the onedimensional and two-dimensional cases are the same.

So the problem considered here is the sedimentation from random initial perturbations in volume fraction at the interface between the particle mixture and the underlying fluid. Specifications of the test problem are given in Table 6 and closely match those for the onedimensional problem.

Figure 25 shows the particle distributions at various times in the transient settling. By $0.2 \mathrm{~s}$, seven, approximately equally spaced structures of downward moving particle-mixture have formed. By $0.4 \mathrm{~s}$, structures have combined, and four major structures exist. The heavy, downward moving particle-mixture falls in spikes, and the lighter fluid rises in rounded bubbles. A kinematic shock appears at the top of the container between the particle mixture and fluid and is seen as a distinct separation between mixture and fluid. The shock moves downward at the same rate as predicted for one-dimensional flow. The mixing region between the particle mixture and pure fluid is defined as the distance between interpenetrating spikes and bubbles. The twodimensional mixing region expands faster than did the one-dimensional interface. By $0.6 \mathrm{~s}$, large spikes of particles extend deep into the fluid, and well formed round bubbles of rising fluid move into the particle-mixture. At $0.8 \mathrm{~s}$, a rising bubble pushes up the top surface of the upper shock, 
and by $1 \mathrm{~s}$, the bubble breaks through the top surface. At $2 \mathrm{~s}$ particles which reached the bottom are sloshing back upward as other particles fall. By $5 \mathrm{~s}$, particles begin to uniformly settle on the bottom to a close-pack state.

This example illustrates the robustness of the MP-PIC method for calculating dense particle flows. The flow is dynamic and particles collide, pack together, and then disperse. The particle field extends from disperse particle flow to high concentration gradients as particles settle to a close-pack state. Using the Eulerian derived stress gradients, the MP-PIC method handles well the complex dynamic packing of particles.

Table 6. Two-dimensional sedimentation problem.

Number of parcels

Number particles in a parcel

Particle radius $(\mathrm{m})$

Particle density $\left(\mathrm{kg} / \mathrm{m}^{3}\right)$

Fluid density $\left(\mathrm{kg} / \mathrm{m}^{3}\right)$

$\mathrm{P}_{\mathrm{s}}(\mathrm{Pa})$

$\theta_{\mathrm{cp}}$

$\beta$

$\mathrm{x}$-gravity, $\mathrm{y}$-gravity $\left(\mathrm{m} / \mathrm{s}^{2}\right)$

Number $x$-cells

Number y-cells

$\mathrm{x}$-domain (m)

y-domain (m)

Fluid viscosity $(\mathrm{kg} / \mathrm{ms})$

Time step (s)

Max residual for $\theta_{\mathrm{p}}, \mathbf{u}_{\mathrm{g}}$ and $\delta \mathrm{p}$
6368

783341

0.001

1000

1

200

0.7

3

$0,-9$

20

40

0.5

1

0.02

0.001

$10^{-7}$

\section{Two-dimensional unstable buoyancy mixing layer}

A sedimentation experiment similar to that described by Youngs (1989) is calculated. The experiment contained a heavy fluid above a light fluid. The test container was accelerated 15 times gravity using a rocket. MP-PIC calculations were made with a particle densities of 3.5, 6, 11, 16 and $26 \mathrm{~kg} / \mathrm{m}^{3}$ and fluid density of $1 \mathrm{~kg} / \mathrm{m}^{3}$. The initial particle volume fraction in the overlying fluid-particle mixture was 0.2 , which gave density ratios of $1.5,2,3,4$ and 6 . The interphase drag coefficient was set to $10^{6} \mathrm{~s}^{-1}$ which locked the particles and fluid together. The experimental test cell was $150 \mathrm{~mm}$ wide with no reported depth. The calculation was made using a container depth of $300 \mathrm{~mm}$. An initial perturbation from particles randomly distributed about a $0.8 \mathrm{~mm}$ amplitude, $13.6 \mathrm{~mm}$ long sine wave was applied to the mixture-fluid interface. Specifications for the calculation are given in Table 7. 
Figure 26 shows the growth of the mixing layer at various times. The particles and fluid form bubbles $(0.04 \mathrm{~s})$ which progress to spikes $(0.08 \mathrm{~s})$ of downward flowing heavy mixture. Youngs (1989) reported that the measured half mixing width, $h$, compared well with

$$
h=\alpha A g t^{2} \text {, }
$$

where the Atwood number is $A=\left(\rho_{1}-\rho_{2}\right) /\left(\rho_{1}+\rho_{2}\right), g$ is gravity, and the constant $\alpha$ lies between 0.05 to 0.07 . Following Youngs (1989), the calculated half mixing-layer width is the distance from the original interface of the deepest downward penetrating structure. An algorithm identifies the deepest structure by ignoring the three deepest particles and then averaging the distances of the next six deepest particles. Similar to experiments (Linden, et al., 1994), the square root of the growth rate curves are adjusted so they pass through the origin. Figure 27 shows that the MP-PIC calculated mixing half-width compares well with the half-width from $h=0.07 A g t^{2}$, which, in turn, agrees well with measured data.

Table 7. Layered sedimentation comparable to experiments by Youngs (1989).

Number of parcels

Particle radius $(\mathrm{m})$

Particle density $\left(\mathrm{kg} / \mathrm{m}^{3}\right)$

Fluid density $\left(\mathrm{kg} / \mathrm{m}^{3}\right)$

$\mathrm{P}_{\mathrm{s}}(\mathrm{Pa})$

$\theta_{\mathrm{cp}}$

$\mathrm{x}$-gravity, y-gravity $\left(\mathrm{m} / \mathrm{s}^{2}\right)$

Number $x$-cells

Number $y$-cells

$\mathrm{x}$-domain (mm)

y-domain (mm)

Time step (s)

Max residual for $\theta_{\mathrm{p}}, \mathbf{u}_{\mathrm{g}}$ and $\delta \mathrm{p}$

Relaxation on $\theta_{\mathrm{p}}, \mathbf{u}_{\mathrm{g}}$ and $\delta \mathrm{p}$

Interphase drag coef. $\mathrm{s}^{-1}$

Initial $\theta_{\mathrm{p}}$
12,000

0.0001

$3.5,6,11,16,26$

1

0

0.7

$0,-147$

25

20

150

300

0.0005

$10^{-8}$

$1,1,1$

$10^{6}$

0.2 

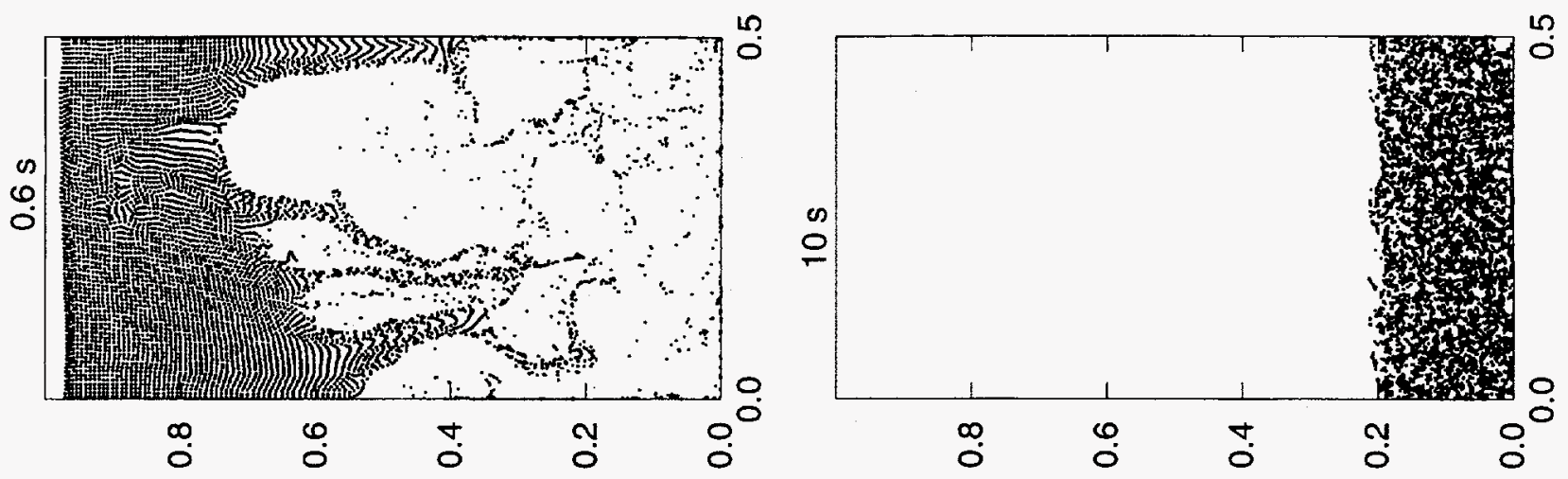

?

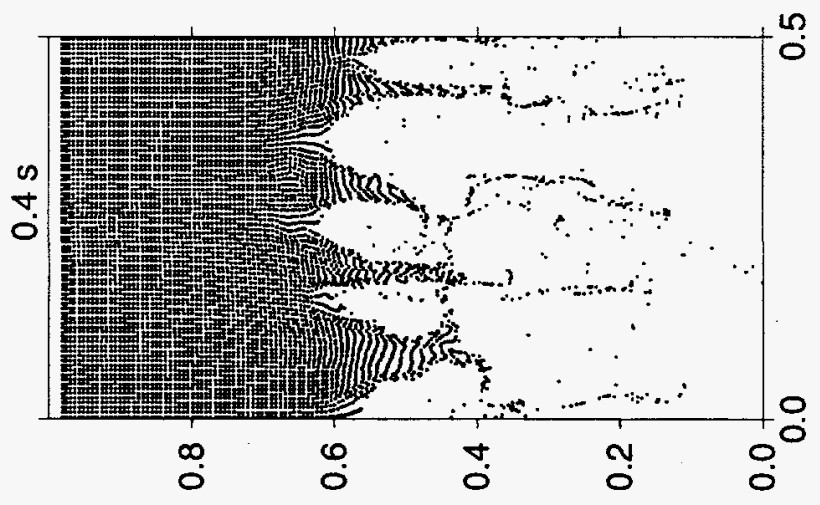

?

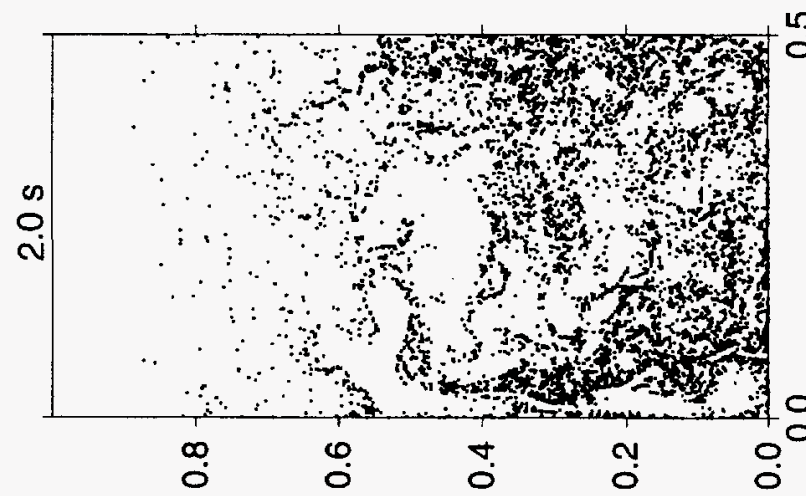

0

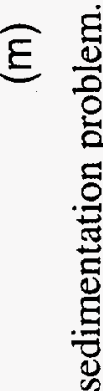
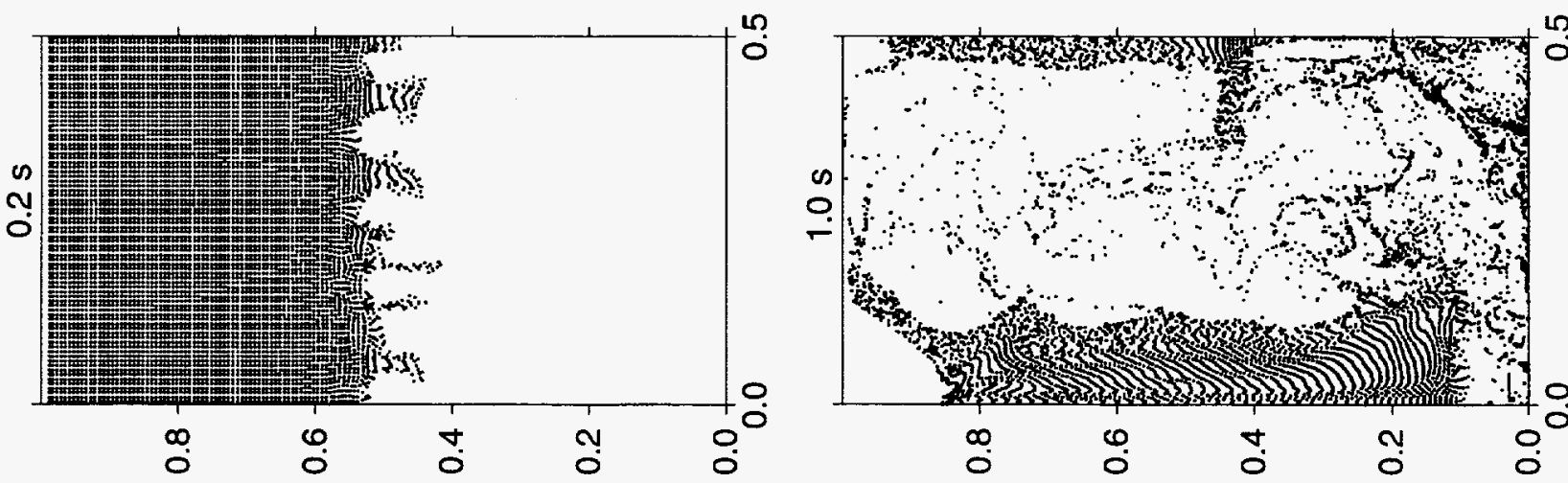

$0^{\circ}$

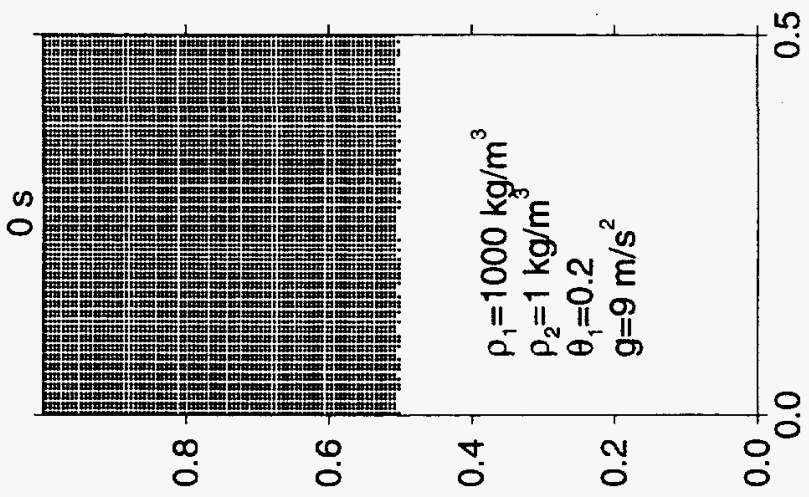

(w)

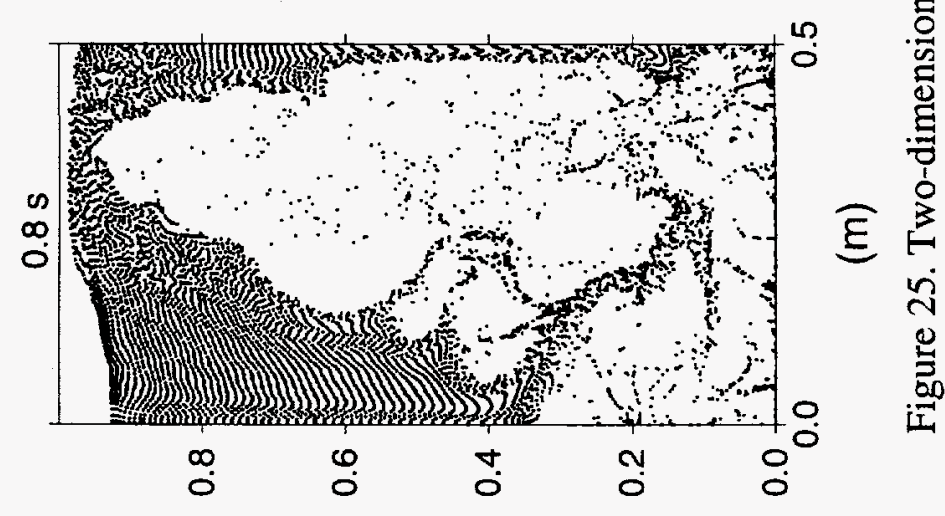

(iv) 

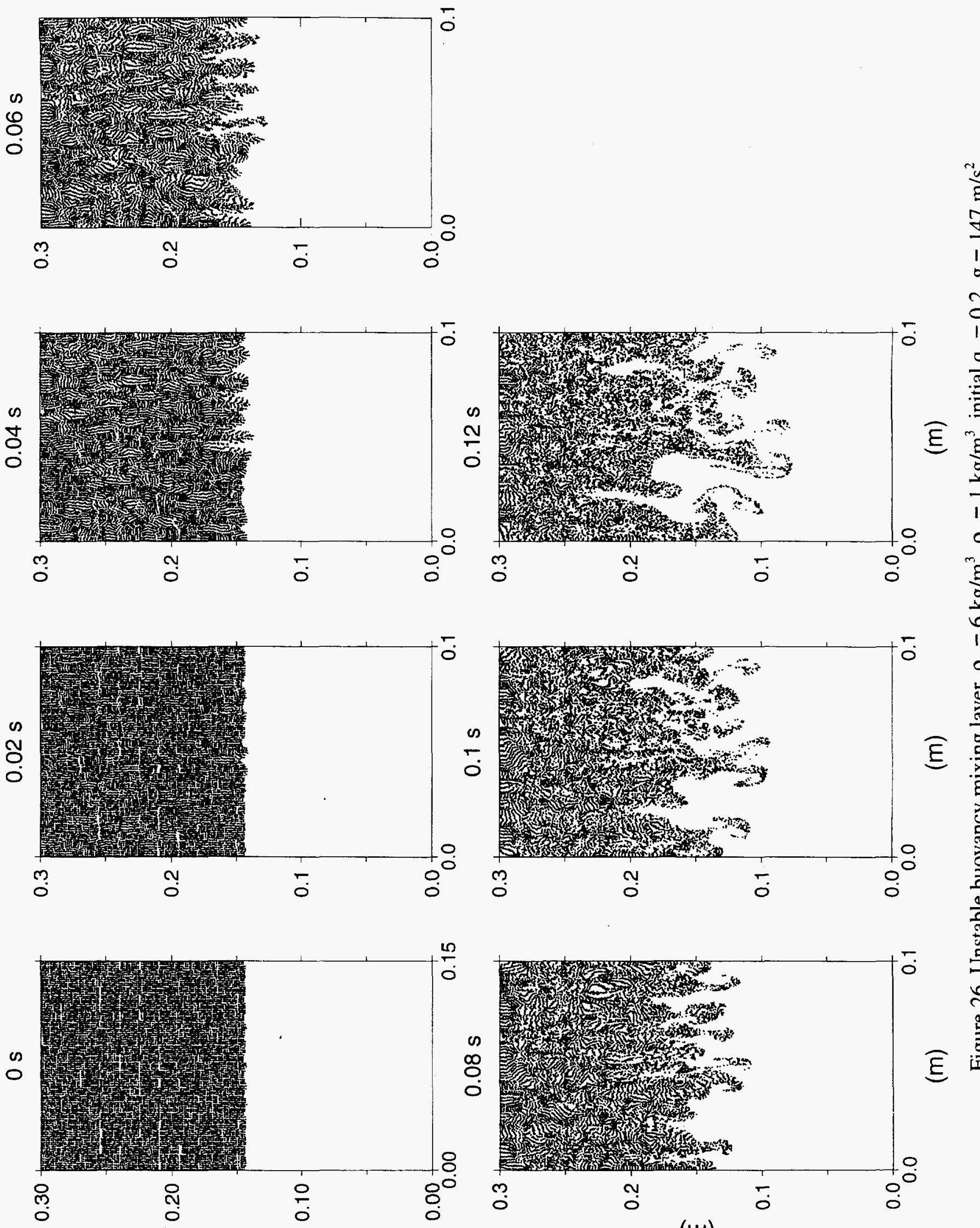

E $\frac{1}{ \pm 0}$

(u)

(u) 


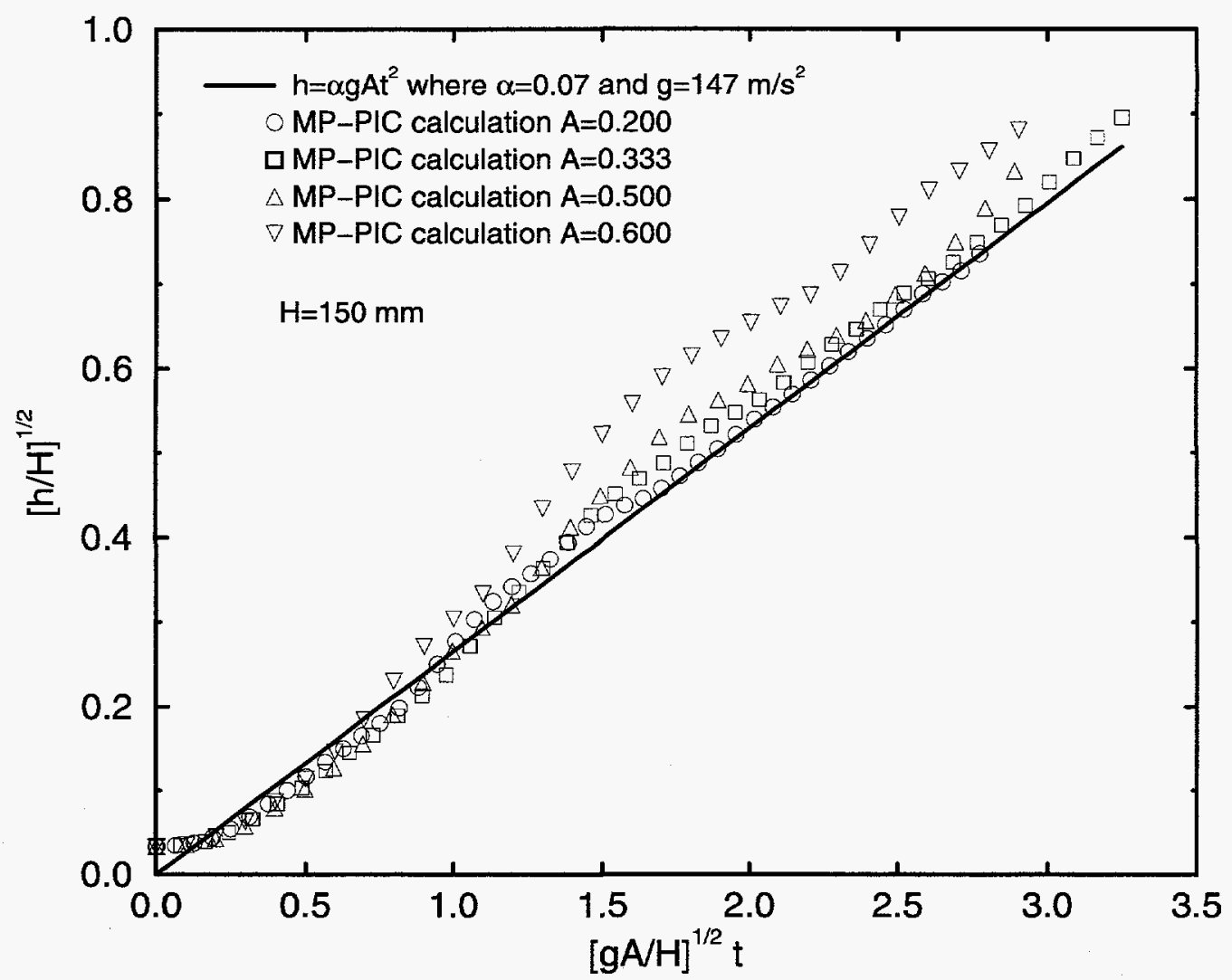

Figure 27. Unstable buoyancy mixing layer growth rate. 


\section{TWO-DIMENSIONAL FLUIDIZED BED}

A gas-particle fluidized bed is formed by rising fluid that lifts and floats particles as illustrated in Figure 28 . The bed can contain a variety of size, shape and density particles. Experimenters have measured the complex circulation of solids and gas, to better understand combustion, mixing, erosion and other bed behavior. The experiments have shown that both the gas and particle flow can be complex with beds pulsing, and bubbles of gas rising through the bed. The fluidized bed experiences dynamic, high particle volume fractions near close-pack, and poses a difficult problem for particle solutions. MP-PIC calculations are made for two fluidized bed experiments to illustrate the ability of the method to predict dynamic, dense particle volume fraction flow.

The first calculation is for experiments by Lin, et al. (1985). Lin, et al., measured the time-average particle velocities and volume fraction in a cylindrical fluidized bed. The second calculation uses conditions from an Argonne National Laboratory IIT experiment reported by Ding and Gidaspow (1990). The IIT experiments were done in a rectangular test channel.

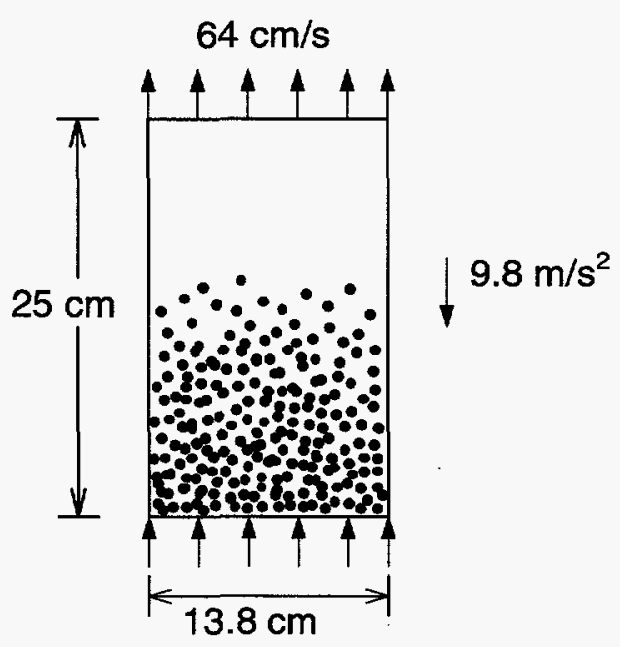

Figure 28. Fluidized bed. Dimensions and gas flow rate are from experiments by Lin, et al. (1985).

\section{A. Fluidized bed calculation for experiment by Lin, et al.}

The experiment by Lin, et al. (1985) is calculated. In the experiment, a cylinder filled with glass beads with diameters ranging from $0.42 \mathrm{~mm}$ to $0.6 \mathrm{~mm}$ is fluidized by air. The reported packed bed height was $11 \mathrm{~cm}$. The gas-alone flow rate was $0.64 \mathrm{~m} / \mathrm{s}$, but the inlet gas velocity was not reported. In the MP-PIC calculation, the container is initially filled with particles at $50 \%$ particle volume fraction to a height of $15 \mathrm{~cm}$, which gives close packing at $11 \mathrm{~cm}$. To match the experiment, the radii of particles have a uniform, random distribution between $0.21 \mathrm{~mm}$ and $0.3 \mathrm{~mm}$. The problem specifications are given in Table 8 . 


\begin{tabular}{|c|c|c|}
\hline \multicolumn{3}{|c|}{$\begin{array}{l}\text { Table 8. Two-dimensional fluidized bed problem with } \\
\text { conditions for the experiment by Lin, et al. (1985). }\end{array}$} \\
\hline & $\mathbf{A}$ & $\mathbf{B}$ \\
\hline Number of parcels & 8,064 & 20,736 \\
\hline Particle radius (mm) & 0.21 to 0.3 & 0.21 to 0.3 \\
\hline Particle density $\left(\mathrm{kg} / \mathrm{m}^{3}\right)$ & 2500 & 2500 \\
\hline Fluid density $\left(\mathrm{kg} / \mathrm{m}^{3}\right)$ & 1.093 & 1.093 \\
\hline $\mathrm{P}_{\mathrm{s}}(\mathrm{Pa})$ & 75 & 75 \\
\hline$\theta_{\mathrm{cp}}$ & 0.7 & 0.7 \\
\hline $\mathrm{x}$-gravity, $\mathrm{y}$-gravity $\left(\mathrm{m} / \mathrm{s}^{2}\right)$ & $0,-9.8$ & $0,-9.8$ \\
\hline Number r-cells (uniform $\Delta \mathrm{r}$ ) & 6 & 12 \\
\hline Number $y$-cells (uniform $\Delta y$ ) & 15 & 30 \\
\hline r-domain (m) & 0.069 & 0.069 \\
\hline y-domain (m) & 0.300 & 0.300 \\
\hline Fluid viscosity (kg/ms) & $1.95 \times 10^{-5}$ & $1.95 \times 10^{-5}$ \\
\hline Time step (s) & 0.002 & 0.001 \\
\hline Max residual for $\theta_{\mathrm{p}}, \mathbf{u}_{\mathrm{g}}$ and $\delta \mathrm{p}$ & $10^{-7}$ & $10^{-7}$ \\
\hline Relaxation on $\theta_{\mathrm{p}}, \mathbf{u}_{\mathrm{g}}$ and $\delta \mathrm{p}$ & $1,1,0.6$ & $1,1,0.6$ \\
\hline Mean gas velocity (gas only) (m/s) & 0.648 & 0.648 \\
\hline
\end{tabular}

Figure 29 shows the calculated particle distribution at various time intervals, where dark areas are dense concentration of particles and light areas within the bed are gas bubbles. Time-labels are rounded to $1 / 100 \mathrm{~s}$ (i.e., label $5.03 \mathrm{~s}$ is $5.025 \mathrm{~s}$ ). In the first time frame, at $5.000 \mathrm{~s}$, a gas bubble pushes a dense layer of particles (seen as the dark band near the interface) to the surface. From $5.025 \mathrm{~s}$ to $5.075 \mathrm{~s}$ the gas bubble bursts through the interface and throws particles upward. During this period, the next dense wave of particles forms deep within the bed as bubbles of gas move from the walls toward the center. From $5.100 \mathrm{~s}$ to $5.225 \mathrm{~s}$ particles fall back into the bed, and the bed collapses onto the next rising wave of particles. At $5.285 \mathrm{~s}$ to $5.340 \mathrm{~s}$, gas bubbles again move from the periphery to the center and rise to the surface similar to the first time frame at $5.000 \mathrm{~s}$. This general cyclic behavior continues in the calculation. 


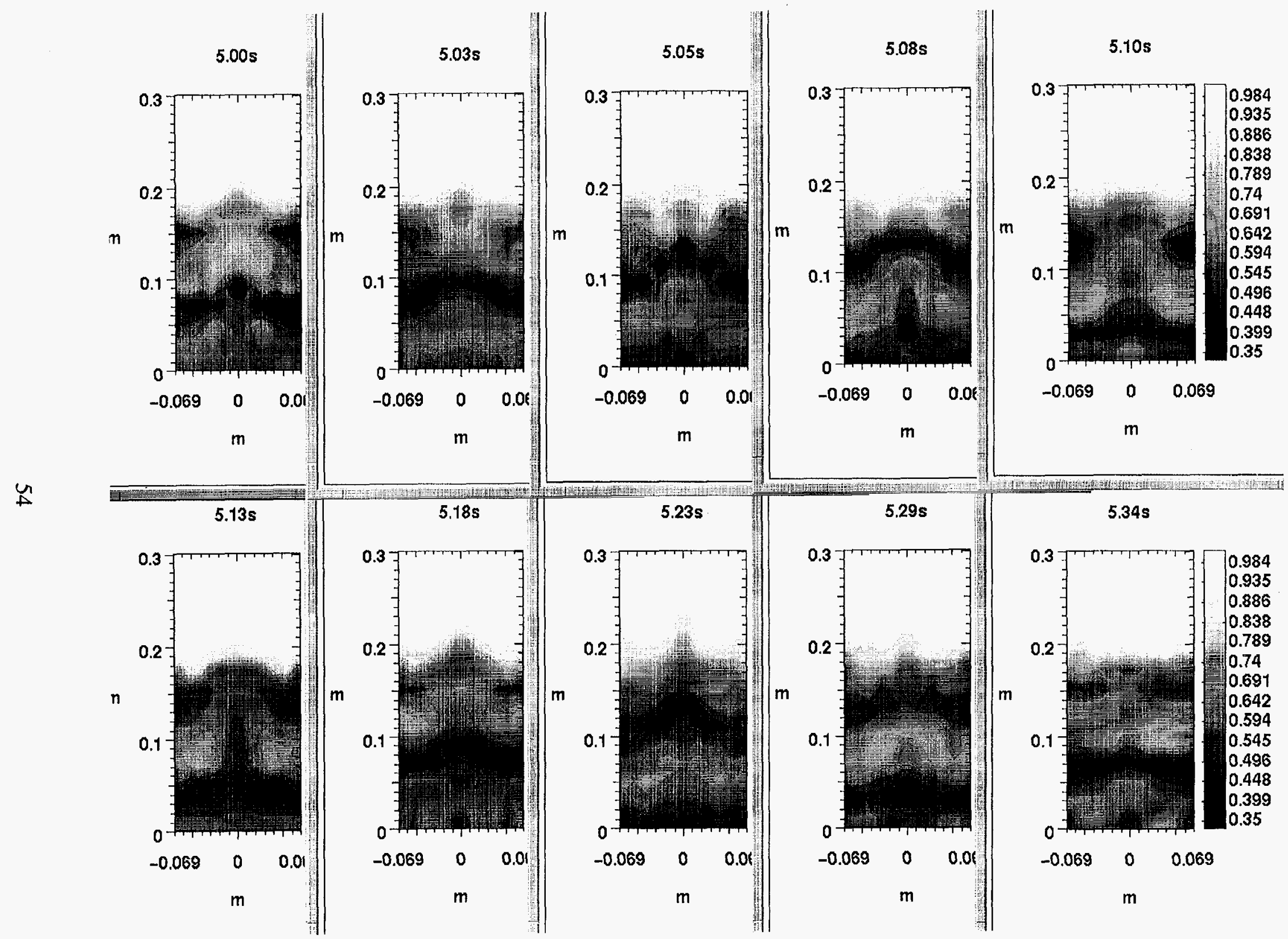

Figure 29. MP-PIC calculated particle volume fraction field at time intervals for the experiment by Lin, et al. (1985). Inlet flow $=64 \mathrm{~cm} / \mathrm{s}$ and $P_{s}=75 \mathrm{~Pa}$. Radial nodes $=6$. Axial nodes $=15$. 
The above calculated behavior compares well with the measured flow by Lin, et al. (1985). Werther and Molerus (1973) described the phenomena in terms of bubble behavior where bubbles rise and move toward the center with increasing height. If the channel is deep enough bubbles would eventually merge at the center. Lin, et al. (1985) present their observed flow behavior by the illustration shown in Figure 30.

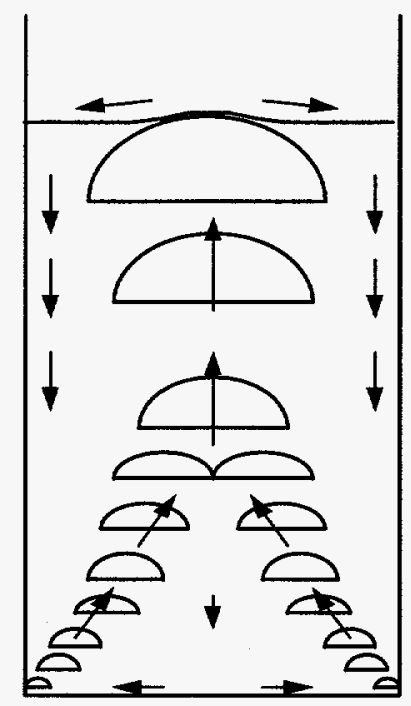

Figure 30. Lin, et al. (1985) illustrated recalculation behavior for high fluidizing velocity.

The experimental flow behavior of both particles and gas was complex (Chao, 1996), and time averaged data for flows and volume fraction were reported by Lin, et al. (1985). The average calculated particle velocity is shown in Figure 31. To obtain the average solids velocity, the particle velocities are mapped to the Eulerian grid using a bilinear interpolation and time averaged for a two second period. Both the measured and calculated average flow pattern shows an upward flow of particles in the center of the pipe and a downward flow of particles near the wall. The magnitude of the MP-PIC calculated average particle velocity matches the measured velocity. The calculation predicts deep circulation patterns of particles moving from the bed surface to the bottom of the bed. Measurements show the strong circulation pattern shown in Figure 31, in the upper region of the bed, but the measured average velocity magnitude is smaller than that calculated at the bottom of the bed. Considering that the fluidized bed calculations are made with the same models used for other unrelated calculations in this study, and that the experimental bed inlet velocity is unknown, and that no special models or correlation for fluidized beds was used, the comparison is remarkably good.

Figure 32 compares the measured particle volume fraction with the MP-PIC calculated instantaneous volume fraction and the calculated average particle volume fraction. The measured volume fraction peaks at $5 \mathrm{~cm}$ which is because of the strong particle circulation pattern being predominantly in the upper part of the bed. The low measured volume fraction seen at the bottom of the bed is presumably from a high injection gas velocity (the gas inlet velocity was not reported). The MP-PIC calculated particle circulation extends to the 
bottom of the bed, and the cyclic rising waves of bubbles and particles give a nearly flat average volume fraction and do not reflect the measured hump. The calculated instantaneous volume fraction fluctuates over a wide range as the bed pulsates, with transient volume fractions approaching close pack at times. However when averaged, the volume fraction is near constant within the bed and trails off to zero at the top of the bed.

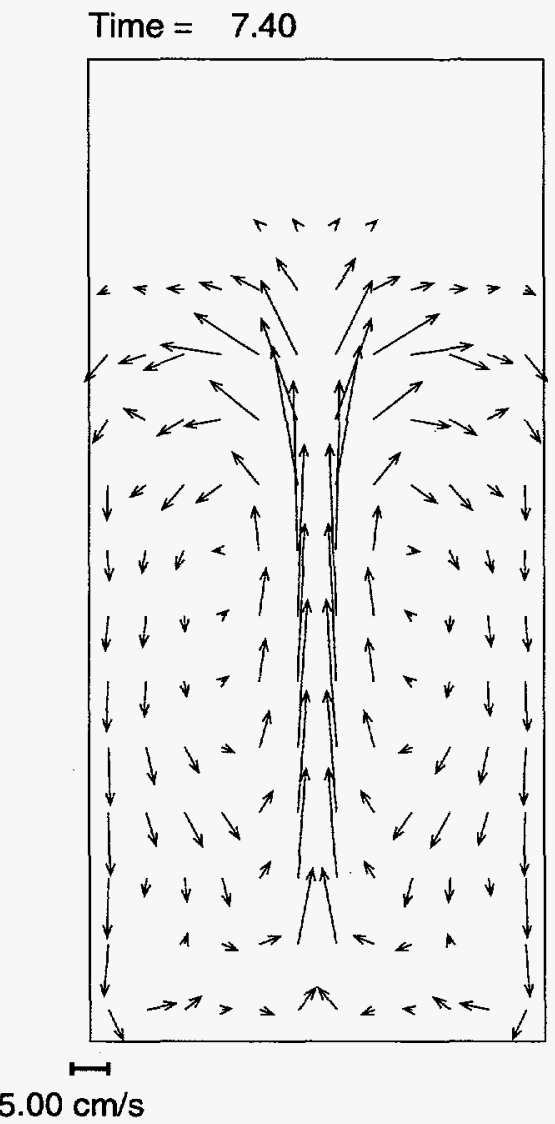

Figure 31. MP-PIC calculated particle velocity field for the experiment by Lin, et al. (1985). Inlet flow $=64 \mathrm{~cm} / \mathrm{s}$ and $P_{s}=75 \mathrm{~Pa}$. Radial nodes $=6$. Axial nodes $=15$. 


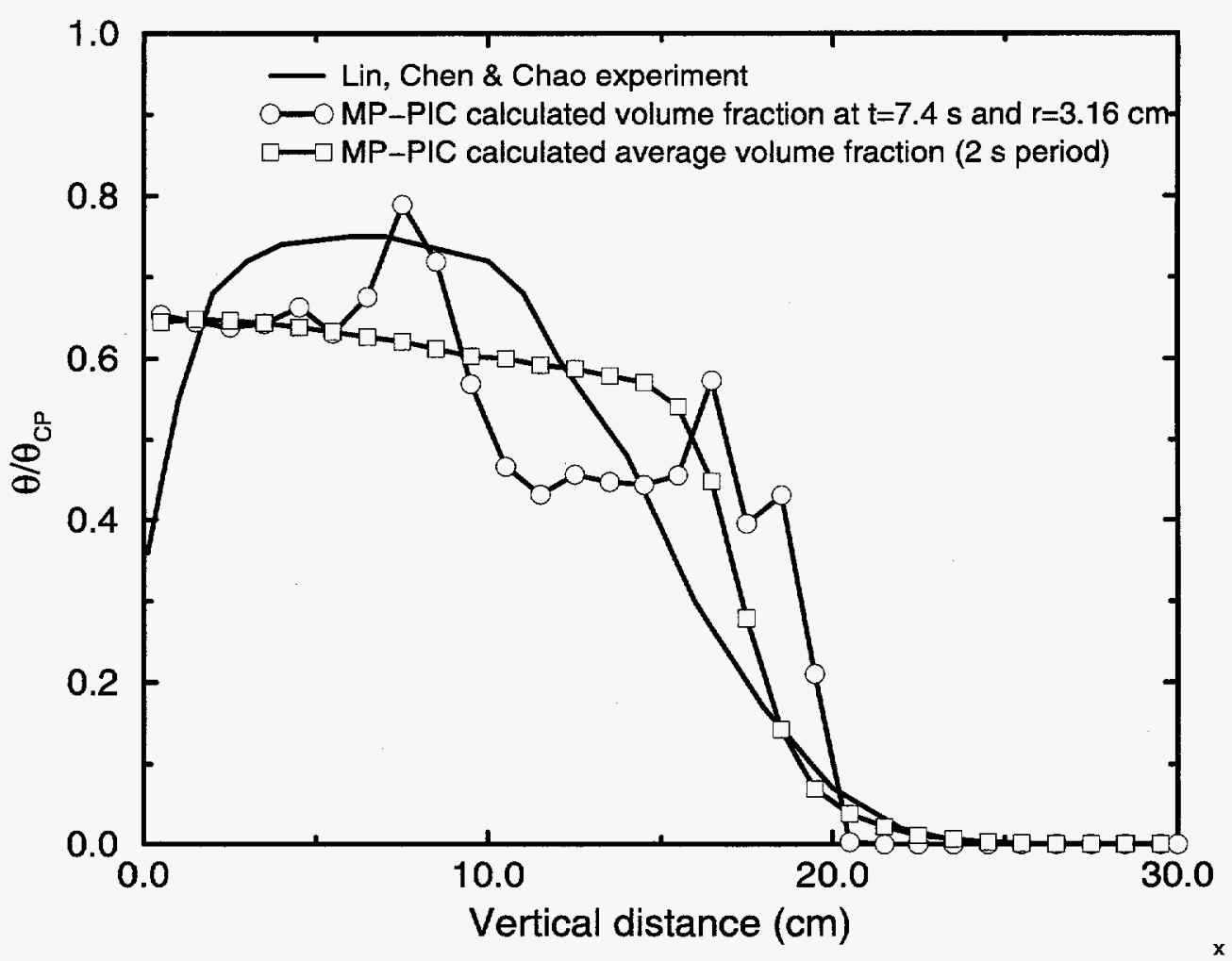

Figure 32. MP-PIC calculated instantaneous and average particle volume fraction compared with that measured by Lin, et al. (1985). Inlet flow $=64 \mathrm{~cm} / \mathrm{s}$ and $P_{\mathrm{s}}=75 \mathrm{~Pa}$. Radial nodes $=6$. Axial nodes $=15$.

\section{Grid independence}

The experiment by Lin, et al. (1985) is calculated in cylindrical coordinates with a constant cell dimension in the radial direction. To check grid independence, two calculations were made. One calculation used 6 radial nodes, 15 axial nodes, and 8,064 particles, and the other calculation used 12 radial nodes, 30 axial nodes, and 20,736 particles. The specification of the calculations is given in Table 8 . The instantaneous time volume fraction field at the finer nodalization is shown in Figure 33. The cyclic pulsing seen in the coarser grid is present in the finer grid calculation with the same period. The finer grid calculation shows a smoother variation in particle concentration gradients.

Figure 34 shows the calculated particle velocity field for the finer nodalization, and Figure 35 shows the average volume fraction for the finer nodalization. Again the low and high resolution calculation provide the same prediction of the fluidized behavior. 

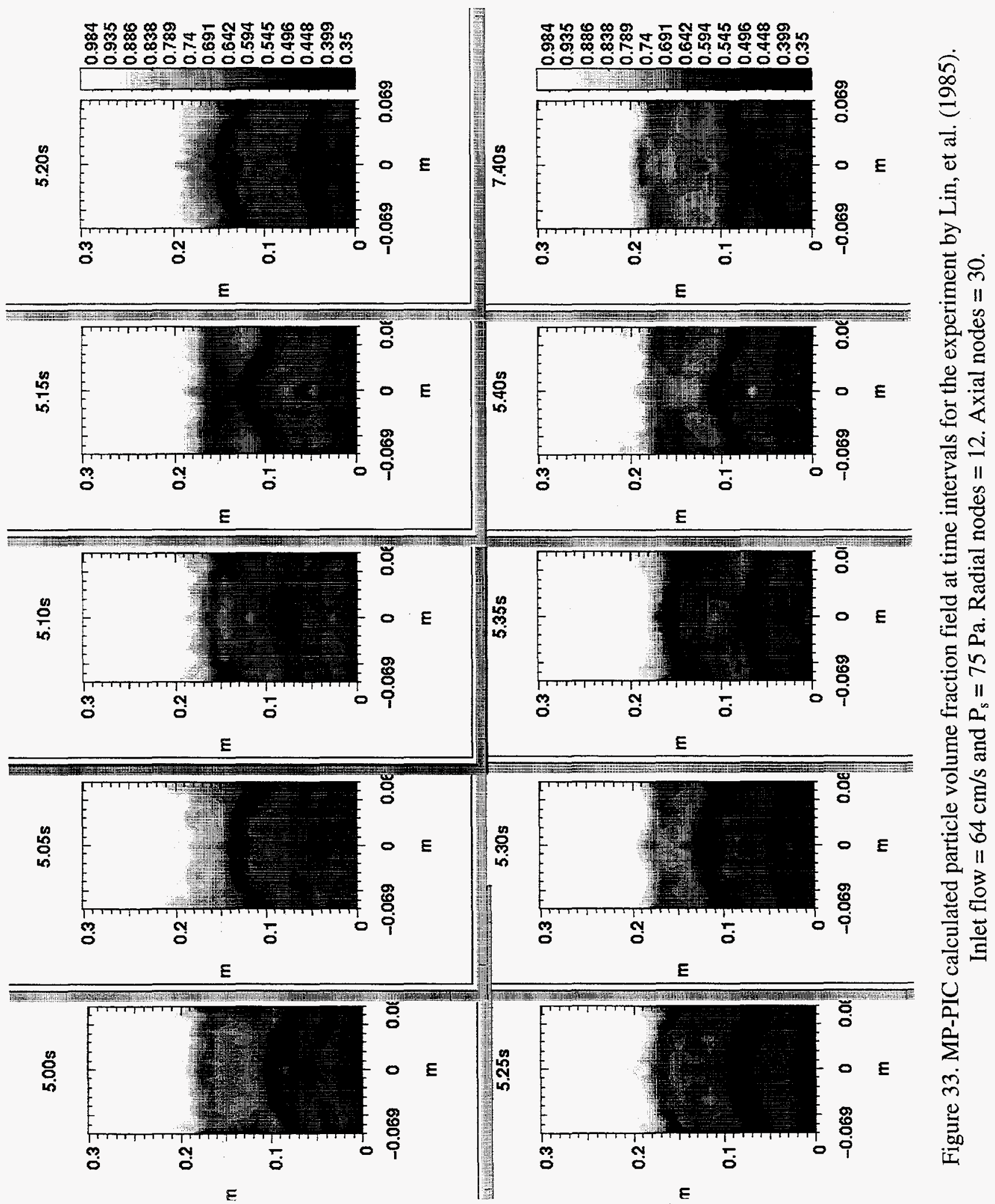

崩 


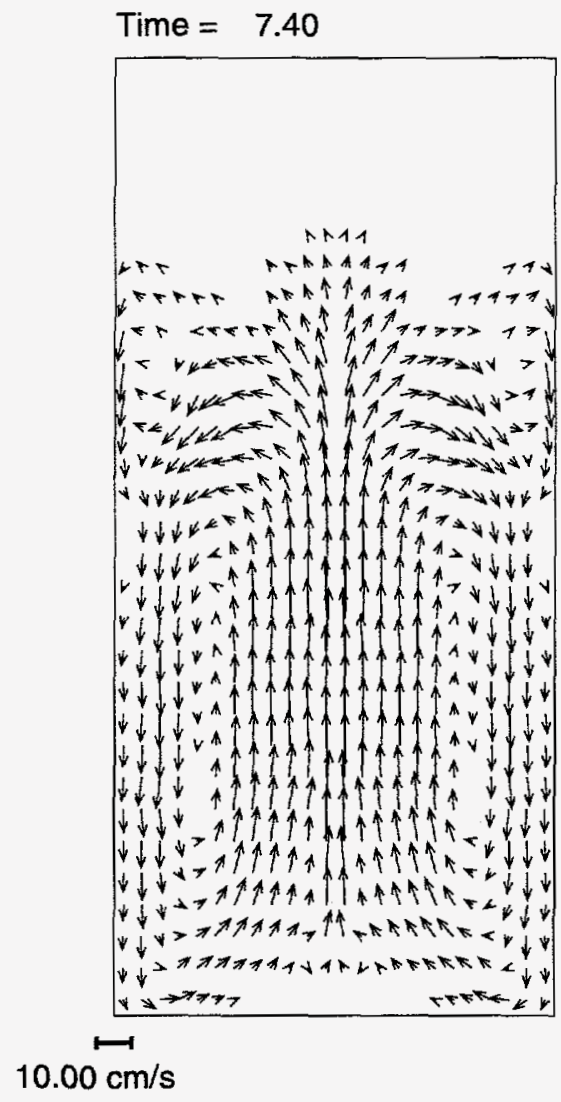

Figure 34. MP-PIC calculated particle velocity field for the experiment by Lin, et al. (1985). Inlet flow $=64 \mathrm{~cm} / \mathrm{s}$ and $P_{\mathrm{s}}=75 \mathrm{~Pa}$. Radial nodes $=12$. Axial nodes $=30$. 


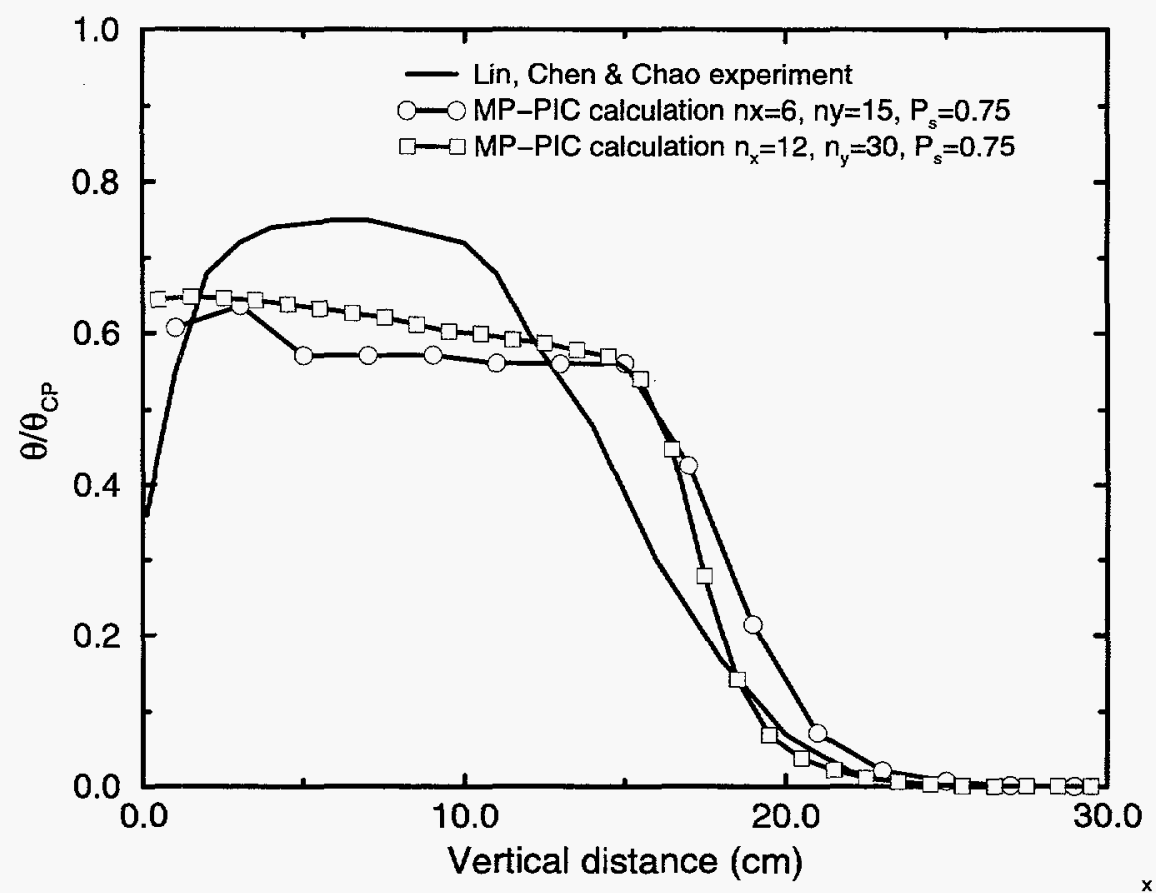

Figure 35. MP-PIC calculated average particle volume fraction compared with that measured by Lin, et al. (1985). Inlet flow $=64 \mathrm{~cm} / \mathrm{s}$ and $P_{s}=75 \mathrm{~Pa}$.

\section{An observation on two-dimensional MP-PIC calculations in cylindrical coordinates}

A particle-in-cell calculation for cylindrical coordinates with uniform spaced radial nodes must have a large number of particle parcels. A few parcels are needed in the small center cell while more and more particle parcels are needed in each succeeding larger outer cell. The experiment by Lin, et al. (1985) had a $6.9 \mathrm{~cm}$ radius cylinder and was filled with $250 \mu \mathrm{m}$ radius particles. With 10 radial nodes and 3 particle parcels per height in the center cell, there must be 57 parcels per height in the outer cell for a uniform radial volume fraction. Increase the nodal resolution to 15 radial cells, and the number of particle parcels in the outer cell increases to 87 parcels. The result is that as the radial node resolution increases, the number of computational parcels dramatically increases.

The number of computational parcels can be reduced by increasing the number of particles within parcels in outer cells. This approach can have devastating effects. A large parcel of particles moving toward the center may not fit into an inner cell. This gives an artificial blockage of particle flow, and because both large and small parcels are blocked (restricted by inner particle stress), center cells become isolated. 


\section{B. Fluidized bed calculation for experiment by Ding and Gidaspow}

An MP-PIC calculation is made for an Argonne National Laboratory IIT experiment which was described by Ding and Gidaspow (1990). Experimental data were not reported but results from a calculation with observed experimental behavior were given. While data provide the only true test of the calculation performance, it is useful to examine the MP-PIC calculation compared with results calculated by Ding and Gidaspow (1990), and their qualitative description of the experiment.

The IIT experiment uses a rectangular flow channel. The calculation begins with the container filled to $50 \mathrm{~cm}$ with a particle-mixture of particle volume fraction 0.4 , which gives close packing at $29 \mathrm{~cm}$. The calculation particles are of one size. The inlet velocity is uniform at the entrance at $100 \mathrm{~cm} / \mathrm{s}$. The specifications of the IIT calculation are given in Table 9.

Ding and Gidaspow (1990) presented an Eulerian numerical calculation of volume fraction and velocity fields for the IIT experiment, and they reported that on-going experiments show that the computed bubbles and flow patterns agree with observed experimental data. They described the bed to surge up with bubbles of fluid rising within the particle-mixture. Their Eulerian calculation predicted the rising fluid bubbles to be symmetric about the bed center line (this symmetry may have been forced on the problem by assuming calculation symmetry about the container center line, although details of the grid were not reported). The MP-PIC calculation predicts cyclic bubbles of gas rising through the bed and bursting through the interface. Figure 36 shows the MP-PIC calculated particle distribution and Figure 37 shows the particle volume fraction mapped to the Eulerian grid. The upward progression of a bubble, from $1.05 \mathrm{~s}$ to $1.25 \mathrm{~s}$, can be followed as it expands the bed. At $1.25 \mathrm{~s}$, the bubble breaks through the surface, and the bed collapses as particles fall back into the bed (from $1.3 \mathrm{~s}$ to $1.4 \mathrm{~s}$ ). This general cyclic expanding and contracting bed behavior was reported for the experiment by Ding and Gidaspow (1990) with the exception that the bubbles from the MP-PIC calculation are not symmetric about the channel center line. Ding and Gidaspow calculated that the particle flow field was also symmetric with particles descending in the center and wall calculation-cells, and symmetric circulation patterns in other cells on the left and right sides of the container. A measured particle velocity magnitude was not reported. Figure 38 shows the MP-PIC average particle velocity fields at times. Particle velocities are mapped to the Eulerian grid and averaged over a $2 \mathrm{~s}$ period prior to the reported time. Non-symmetric circulation patterns are calculated which change with time. Figure 39 shows the gas velocity pattern also changes with time suggesting instabilities are driving the two-dimensional bed flow patterns. To determine whether the instabilities are physical or are manifested from the numeric solution requires experimental data. 


\begin{tabular}{lc}
\hline \hline Table 9. IIT two-dimensional fluidized bed problem. \\
\hline & \\
Number of parcels & 8,000 \\
Particle radius $(\mathrm{mm})$ & 0.25 \\
Particle density $\left(\mathrm{kg} / \mathrm{m}^{3}\right)$ & 2500 \\
Fluid density $\left(\mathrm{kg} / \mathrm{m}^{3}\right)$ & 1 \\
$\mathrm{P}_{\mathrm{s}}(\mathrm{Pa})$ & 100 \\
$\theta_{\mathrm{cp}}$ & 0.7 \\
$\mathrm{X}$-gravity, y-gravity $\left(\mathrm{m} / \mathrm{s}^{2}\right)$ & $0,-9.8$ \\
Number x-cells & 15 \\
Number y-cells & 30 \\
x-domain $(\mathrm{m})$ & 0.4 \\
y-domain $(\mathrm{m})$ & 1 \\
Fluid viscosity $(\mathrm{kg} / \mathrm{ms})$ & $1.9 \times 10^{-5}$ \\
Time step $(\mathrm{s})$ & 0.0005 \\
Max residual for $\theta_{\mathrm{p}}, \mathbf{u}_{\mathrm{g}}$ and $\delta \mathrm{p}$ & $10^{-7}$ \\
Relaxation on $\theta_{\mathrm{p}}, \mathbf{u}_{\mathrm{g}}$ and $\delta \mathrm{p}$ & $1,1,0.6$ \\
Inlet fluid velocity $(\mathrm{m} / \mathrm{s})$ & 1 \\
\hline \hline
\end{tabular}


$1.15 \mathrm{~s}$

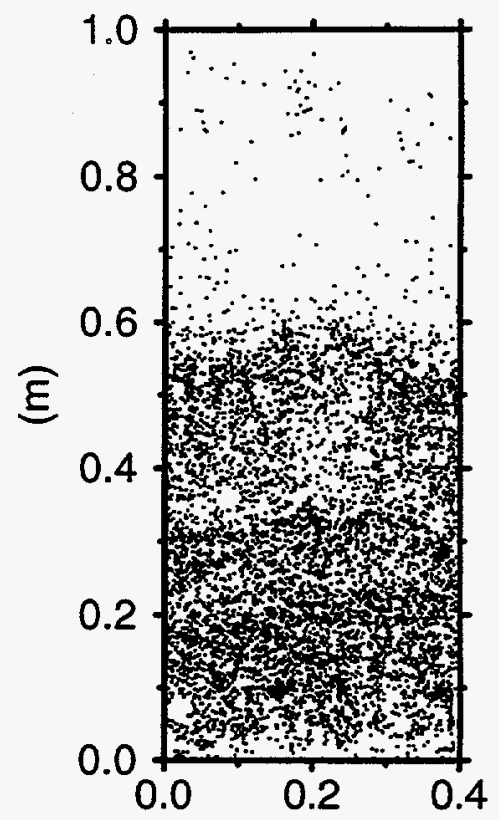

(m)

$1.35 \mathrm{~s}$

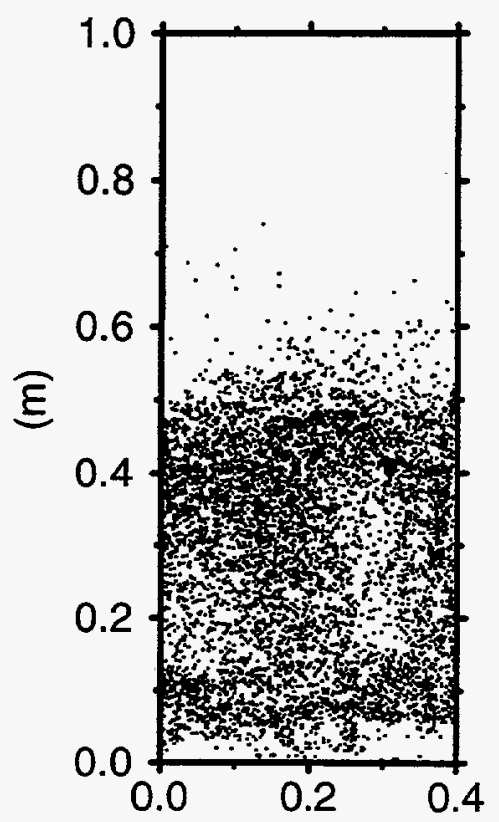

(m)
$1.20 \mathrm{~s}$

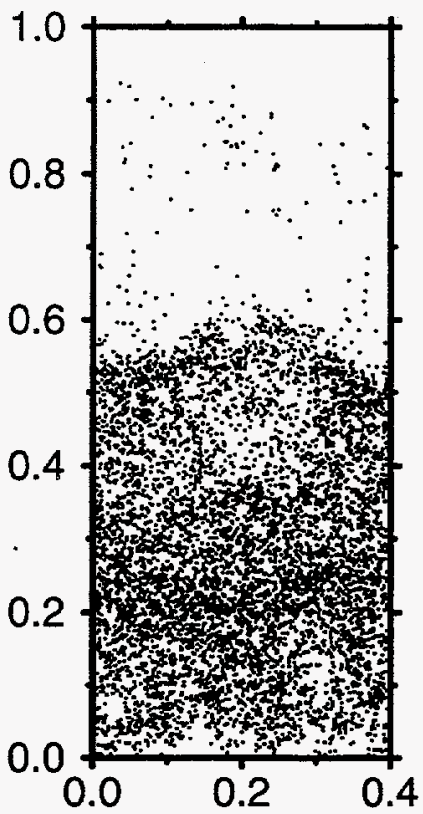

(m)

$1.40 \mathrm{~s}$

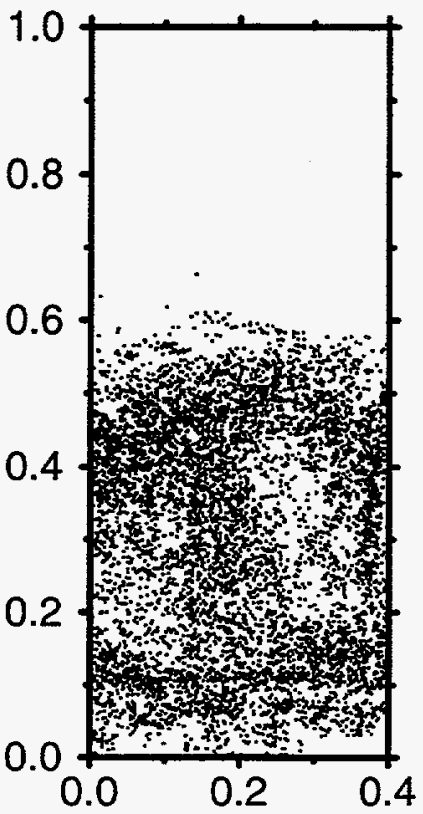

(m)
$1.25 \mathrm{~s}$

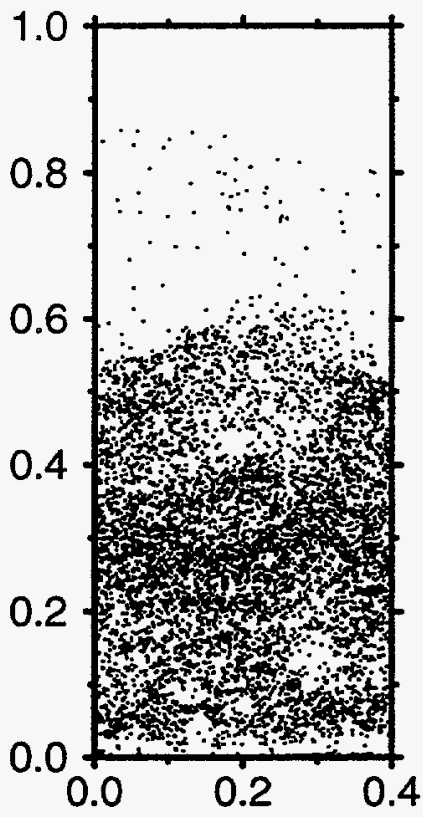

(m)

$1.45 \mathrm{~s}$

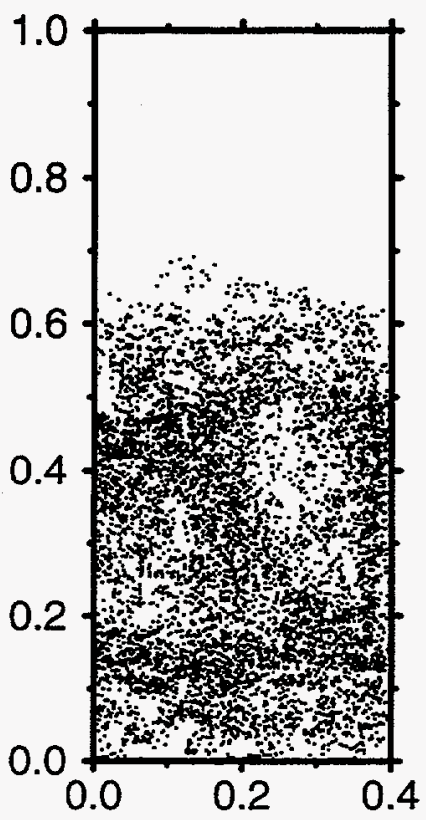

(m)
$1.30 \mathrm{~s}$

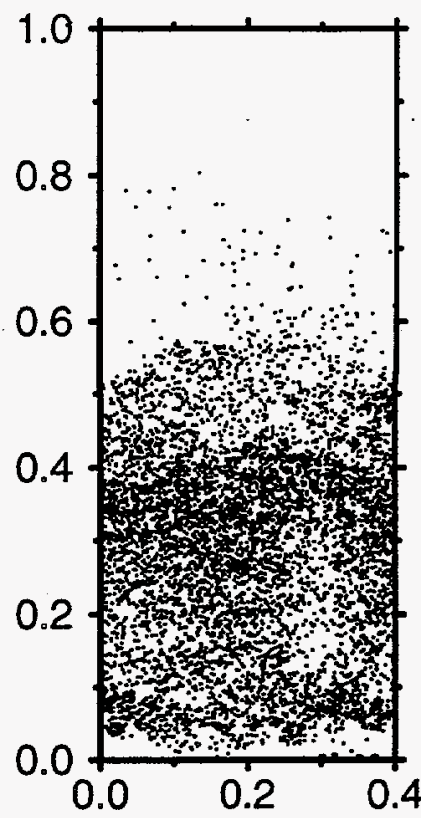

(m)

$1.50 \mathrm{~s}$

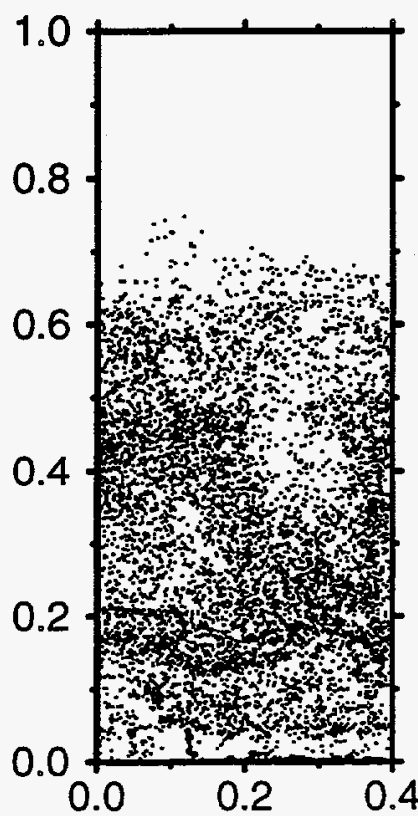

(m)

Figure 36. Particle distribution in the IT fluidized bed. Inlet flow $=1 \mathrm{~m} / \mathrm{s}, \theta_{\mathrm{i}}=0.4$. 


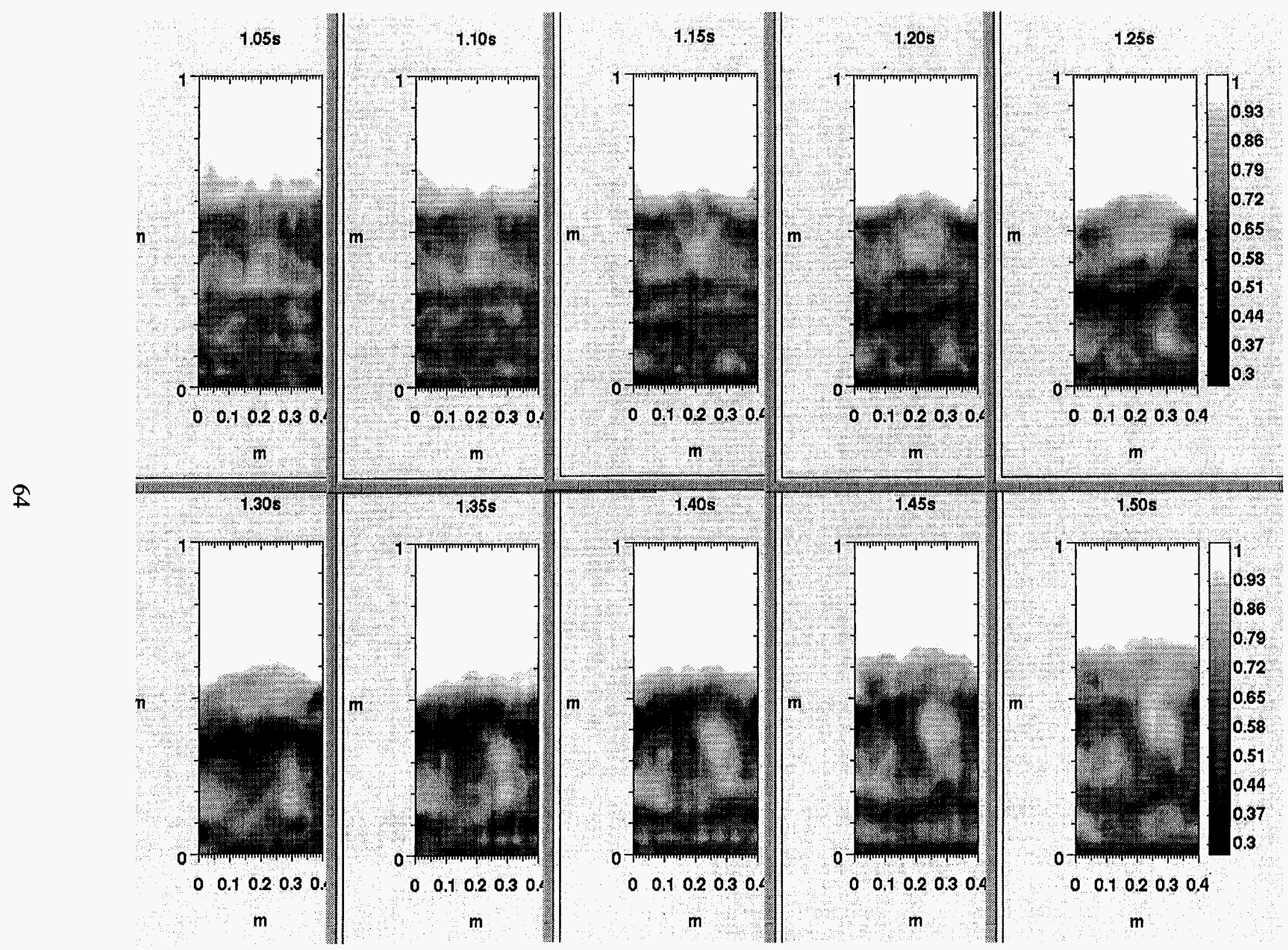

Figure 37. Particle volume fraction field in the IIT fluidized bed. Inlet flow $=1 \mathrm{~m} / \mathrm{s}, \theta_{\mathrm{i}}=0.4$. 

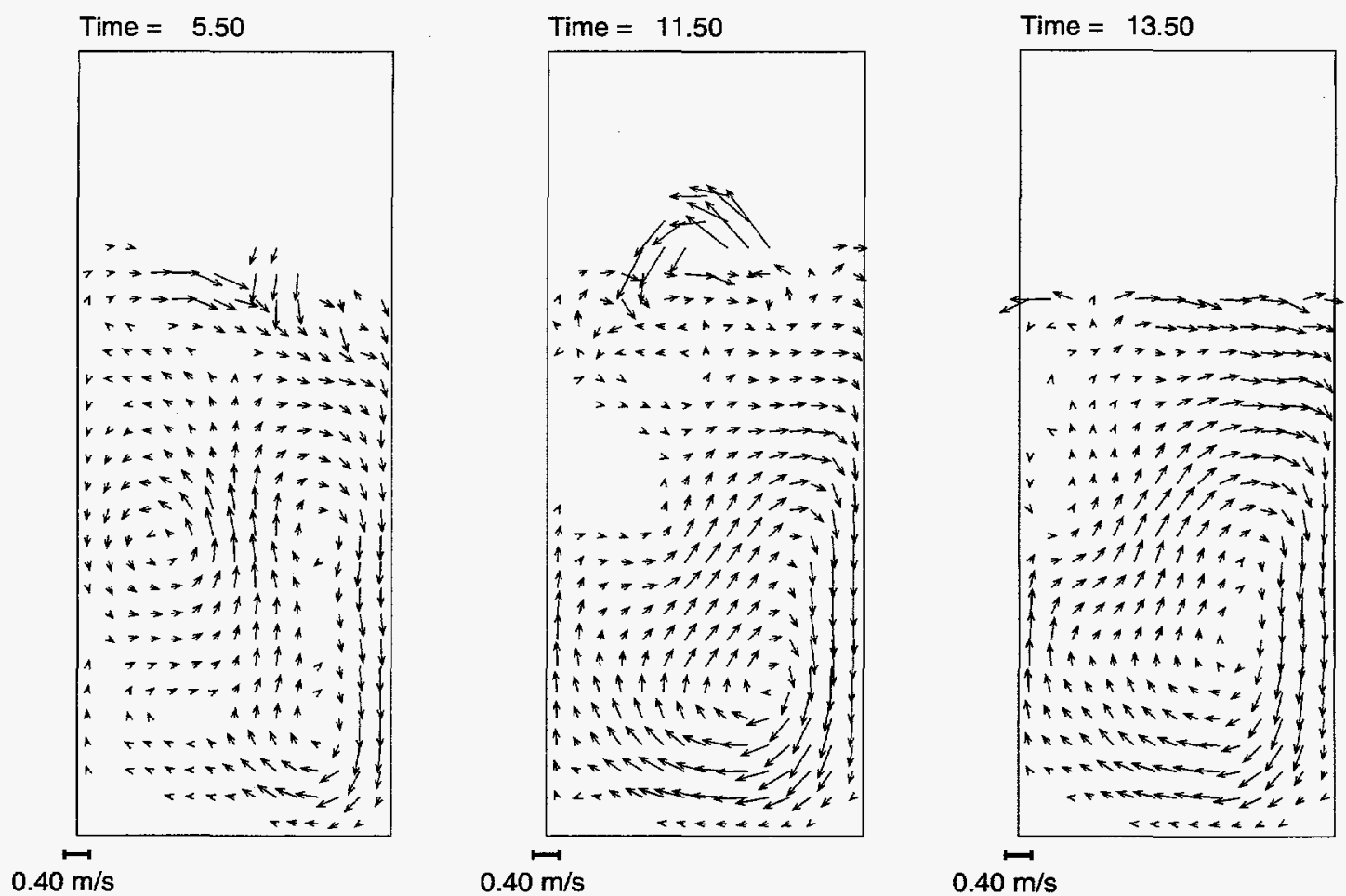

Figure 38. IT fluidized bed average particle velocities. Inlet flow $=100 \mathrm{~cm} / \mathrm{s}, \theta_{\mathrm{i}}=0.3$.
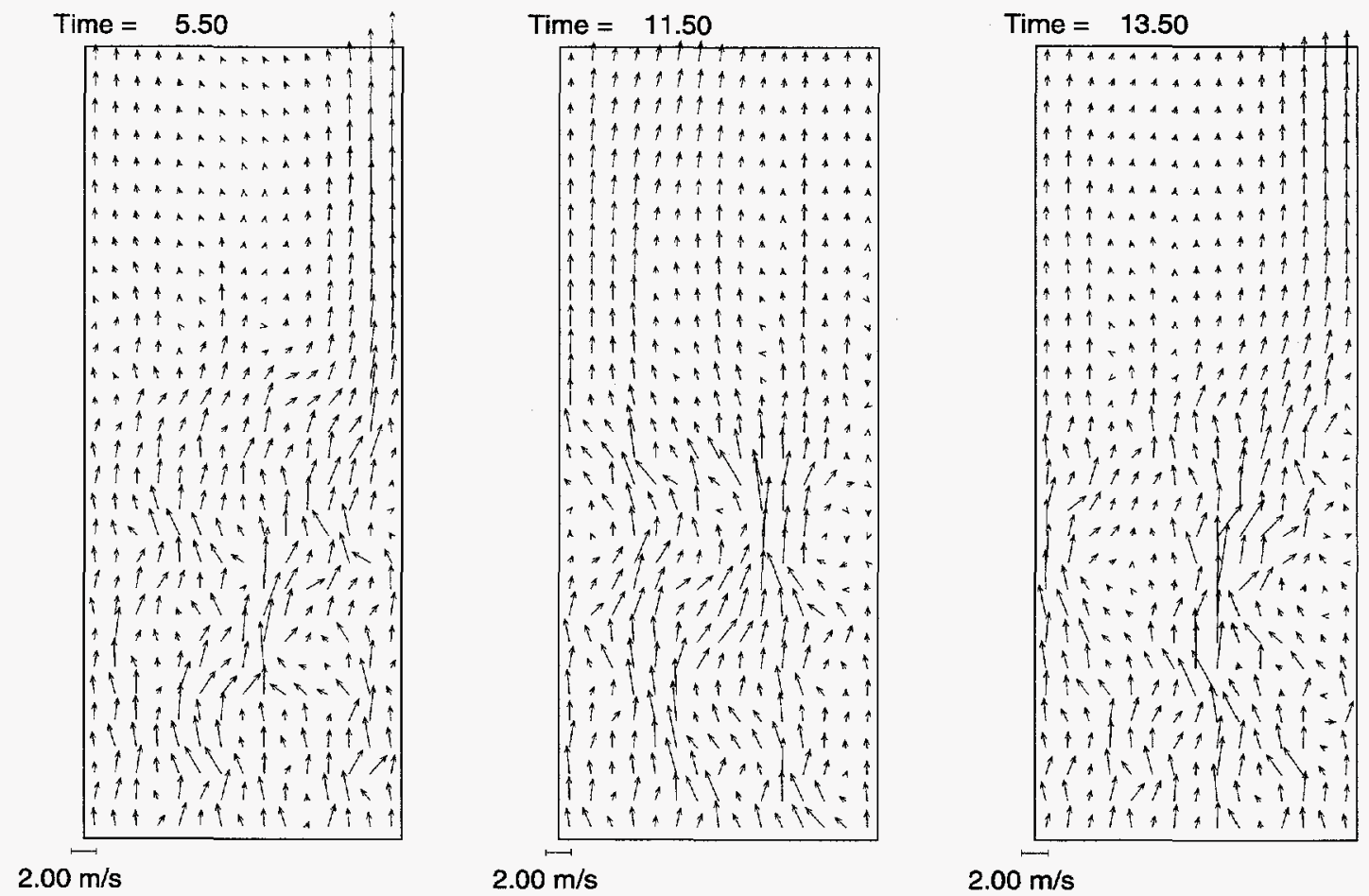

Figure 39. IIT fluidized bed gas velocities. Inlet flow $=100 \mathrm{~cm} / \mathrm{s}, \theta_{\mathrm{i}}=0.3$. 


\section{HOMOGENOUS BATCH SEDIMENTATION}

\section{A. Background}

The separation of particles from a fluid by means of gravity is an important step in many industrial processes as well as in the environment. Homogenous, batch sedimentation begins with a uniform distribution of particles in a container. Let sit, particles separate into regions depending on particle density and size as illustrated in Figure 40 for a bimodal suspension. A suspension with a variety of particle sizes and densities will form regions, where each region above the previous region contains one less species. The top of the vessel will be clear fluid (providing particle densities are greater than the fluid density) and the bottom of the vessel will contain all species. In cases where there is a large variation between sizes and/or density of species, the regions are distinct and separated by strong concentration gradients ("shocks"). A monodisperse mixture containing one size and density particle forms a single region of suspended particles under a clear fluid. Davis and Acrivos (1985) and Al-Naafa' and Selim (1989) provide reviews of experiments and analysis of polydisperse suspensions. Richardson and Zaki (1954) gave an empirical equation for the settling velocity of a monodisperse mixture which compares well with data. The settling velocity is

$$
U_{s}=U_{o}\left(1-\theta_{p}\right)^{n-1}
$$

where $U_{o}$ is the terminal velocity, $\theta_{p}$ is the particle volume fraction and $n$ is a constant on the order of 5. Mirza and Richardson (1978) extended the empirical relation to polydisperse suspensions. Al-Naafa' and Selim (1989), using conservation of mass and empirical settling velocities, provided an analytical model that predicts well the settling of polydisperse suspensions. A calculation by Shih, et al. (1987) for bimodal settling using a one-dimensional, multi-fluid, numerical solution compared well with experimental data, and Andrews and O'Rourke (1996) presented an accurate prediction of polydisperse settling of a suspension using a one-dimensional MP-PIC method.

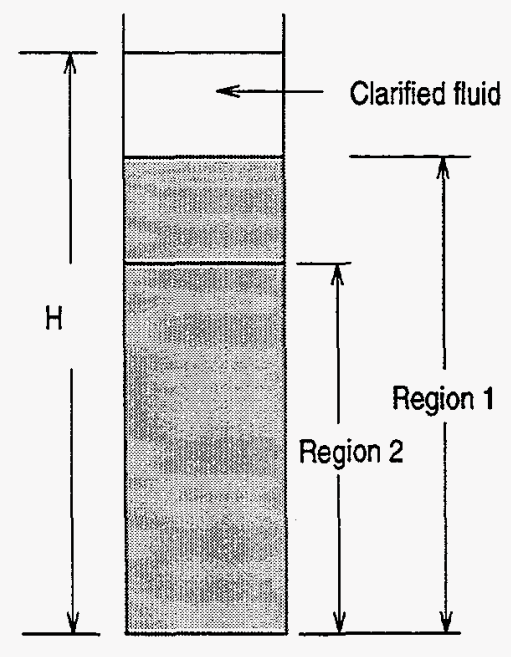

lag19

Figure 40. Illustration of a vertical bimodal suspension. 
Boycott (1920) reported that by tilting the container, corpuscles separated from blood quicker than when the container was vertical. The settling in a tilted container is illustrated in Figure 41. Position $A$ in Figure 41 is the distinct horizontal interface between the clear fluid and mixture $(B)$. The initial height of the mixture is $H$, the transient height of the interface is $h$ and the vessel width is $b$. A thin layer of upward flowing clear fluid forms $(C)$ at the downward-facing surface, and concentrated particles flow down the upward-facing surface $(D)$. Many investigators have studied tilted container phenomena and have provided analytical models for the settling. Davis and Acrivos (1985) and Kapoor and Acrivos (1995) provide reviews of inclined sedimentation. Ponder (1925) and Nakumura \& Kuroda (1937) postulated that for tilted containers the horizontal mixture interface and the interface under the downward facing surface fall at the same mixture velocity as for a vertical container. The observed thin fluid film under the downward facing surface was added directly to the clear fluid above the horizontal mixture layer in the analytical model. The settling rate is

$$
d h / d t=-U_{o}[1+(h / b) \sin \alpha],
$$

where $\alpha$ is the angle of inclination and $U_{o}$ is the mixture interface velocity for a vertical vessel. Acrivos \& Herbolzheimer (1979) showed that the simple settling model given by Ponder, et al. was accurate for a monodisperse suspension, at low Reynolds number, with an initial uniform particle distribution, a large Grashoff number when compared with the Reynolds number, and a stable interface between clear fluid and suspension. Kinosita (1949) noted that particles in a tilted container did not settle through a quiescent fluid as in a vertical container, but that strong convective currents can form a vortex in the suspended phase with particle velocities as high as 100 times the sedimentation velocities. Instabilities have been observed at the interface between the clear fluid flowing up under the downward-facing surface and the particle mixture. Photographs by Herbolzheimer (1983) (see Figure 50) show Kelvin-Helmholtz waves at the interface.

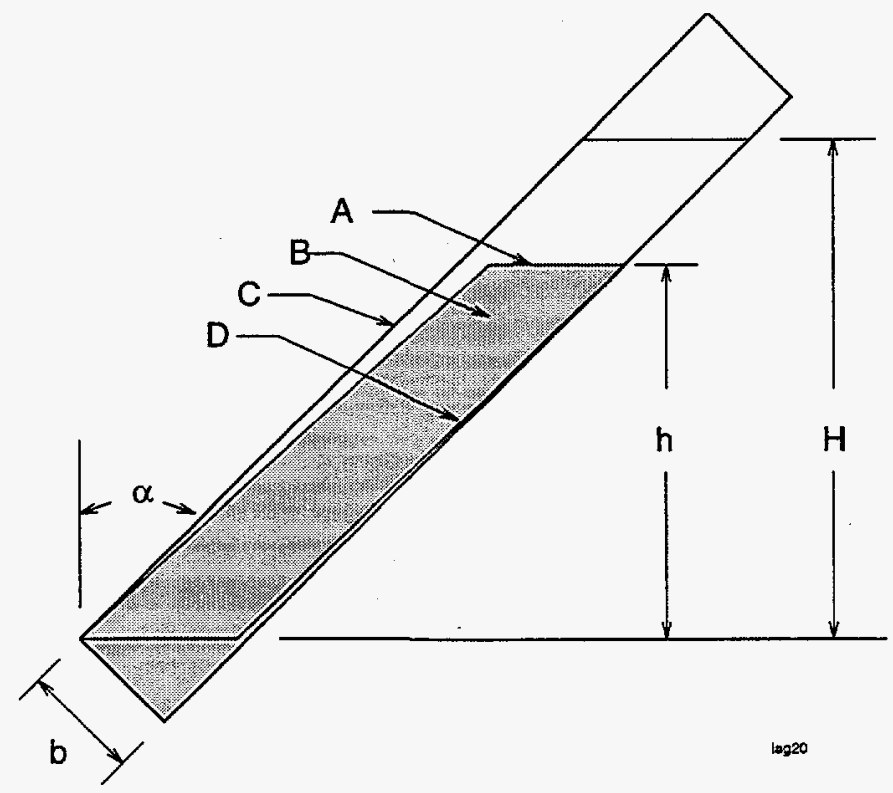

Figure 41. Illustration of a tilted vessel suspension. 


\section{B. MP-PIC solution}

The MP-PIC numerical model has an advantage over prior analytical methods of being able to model flows with an unlimited number of different size and density particles. Because the MPPIC method solves the fundamental governing partial differential equations, fluid currents in the mixture and instabilities at interfaces can be calculated. Furthermore, as a part of numerical solutions, complex boundary and initial conditions can be applied. Examples are presented for vertical and inclined containers and for particles with a range of sizes and densities, and results are compared with measured data.

The sedimentation calculations were for tall, narrow containers which required computational cell height to width ratios as large as 10 to 1 . Particles are small giving a high interphase drag $\left(D_{p}\right.$ $>12,000 \mathrm{~s}^{-1}$ ) and the mean flow in vertical containers is nearly zero.

The two-dimensional MP-PIC calculation gives non-physical currents in vertical batch sedimentation when using the implicit, new-time particle velocity (equation 34),

$$
\mathbf{u}_{p}^{n+1}=\frac{\mathbf{u}_{p}^{n}+\Delta t\left[D_{p} \mathbf{u}_{g, p}^{n+1}-\frac{1}{\rho_{p}} \nabla p_{p}^{n+1}-\frac{1}{\rho_{p} \theta_{p}} \nabla \tau_{p}^{n+1}+\mathbf{g}\right]}{1+\Delta t D_{p}}
$$

substituted into the continuum interphase momentum transfer term (equation 40),

$$
\mathbf{F}_{i+1 / 2, j}^{n+1}=\sum_{p} S_{i+1 / 2, j}\left[D_{p}\left(\mathbf{u}_{g, p}^{n+1}-\mathbf{u}_{p}^{n+1}\right)-\frac{1}{\rho_{p}} \nabla p_{p}^{n+1}\right] N_{p} m_{p} .
$$

The new-time particle velocity substituted into the interphase momentum transfer equation gives equation (41). Using the old-time particle velocity, $\mathbf{u}_{p}^{n}$, the non-physical currents are eliminated, and the settling is calculated well. This is contrary to common wisdom, where implicit schemes usually add stability to the solution. No time step limitations were experienced using the old-time particle velocities. The stability issue is being examined and is not presented in this study. The batch sedimentation calculations use the old-time particle velocity in $F$, and in the following sections, the calculations are shown to give good agreement with experimental data.

\section{Polydisperse suspensions}

An MP-PIC calculation of the bimodal suspension of a glass beads experiment given by Davis, et al. (1982) is illustrated in Figure 40. The fluid density and viscosity are $992 \mathrm{~kg} / \mathrm{m}^{3}$ and $0.0667 \mathrm{~kg} /(\mathrm{m}-\mathrm{s})$, respectively. The small glass bead density is $2440 \mathrm{~kg} / \mathrm{m}^{3}$ and beads range in diameter from 125 to $150 \mu \mathrm{m}$. The larger bead density is $2990 \mathrm{~kg} / \mathrm{m}^{3}$ and beads range in diameter from 177 to $219 \mu \mathrm{m}$. The calculation uses a uniform random distribution of particle sizes between the reported experimental ranges. Initial volume fractions for the small and large beads are $3 \%$ and $1 \%$, respectively. Specifications for the calculation are given in Table 10. 
Table 10. Batch bimodal sedimentation

(Davis, et al., 1982).

Tilt

$0^{\circ}$

Number of parcels particle 1

8,000

Number of parcels particle 2

8,000

Initial volume fraction particle 1

0.03

Initial volume fraction particle 2

0.01

Particle 1 radius $(\mu \mathrm{m})$

63

Particle 2 radius $(\mu \mathrm{m})$

89

Particle 1 density $\left(\mathrm{kg} / \mathrm{m}^{3}\right)$

2440

Particle 2 density $\left(\mathrm{kg} / \mathrm{m}^{3}\right)$

2990

Fluid density $\left(\mathrm{kg} / \mathrm{m}^{3}\right)$

992

$\mathrm{P}_{\mathrm{s}}(\mathrm{Pa})$

50

$\theta_{\mathrm{cp}}$

0.6

$\mathrm{x}$-gravity, y-gravity $\left(\mathrm{m} / \mathrm{s}^{2}\right)$

Number $x$-cells

$0,-9.8$

Number y-cells

5

$\mathrm{x}$-domain $(\mathrm{cm})$

40

$y$-domain $(\mathrm{cm})$

5

100

Fluid viscosity $(\mathrm{kg} / \mathrm{m}-\mathrm{s})$

0.0667

Time step (s)

0.5

Max residual for $\theta_{\mathrm{p}}, \mathbf{u}_{\mathrm{g}}$ and $\delta \mathrm{p}$

$10^{-10}$

Relaxation on $\theta_{\mathrm{p}}, \mathbf{u}_{\mathrm{g}}$ and $\delta \mathrm{p}$

$1,1,0.5$ 

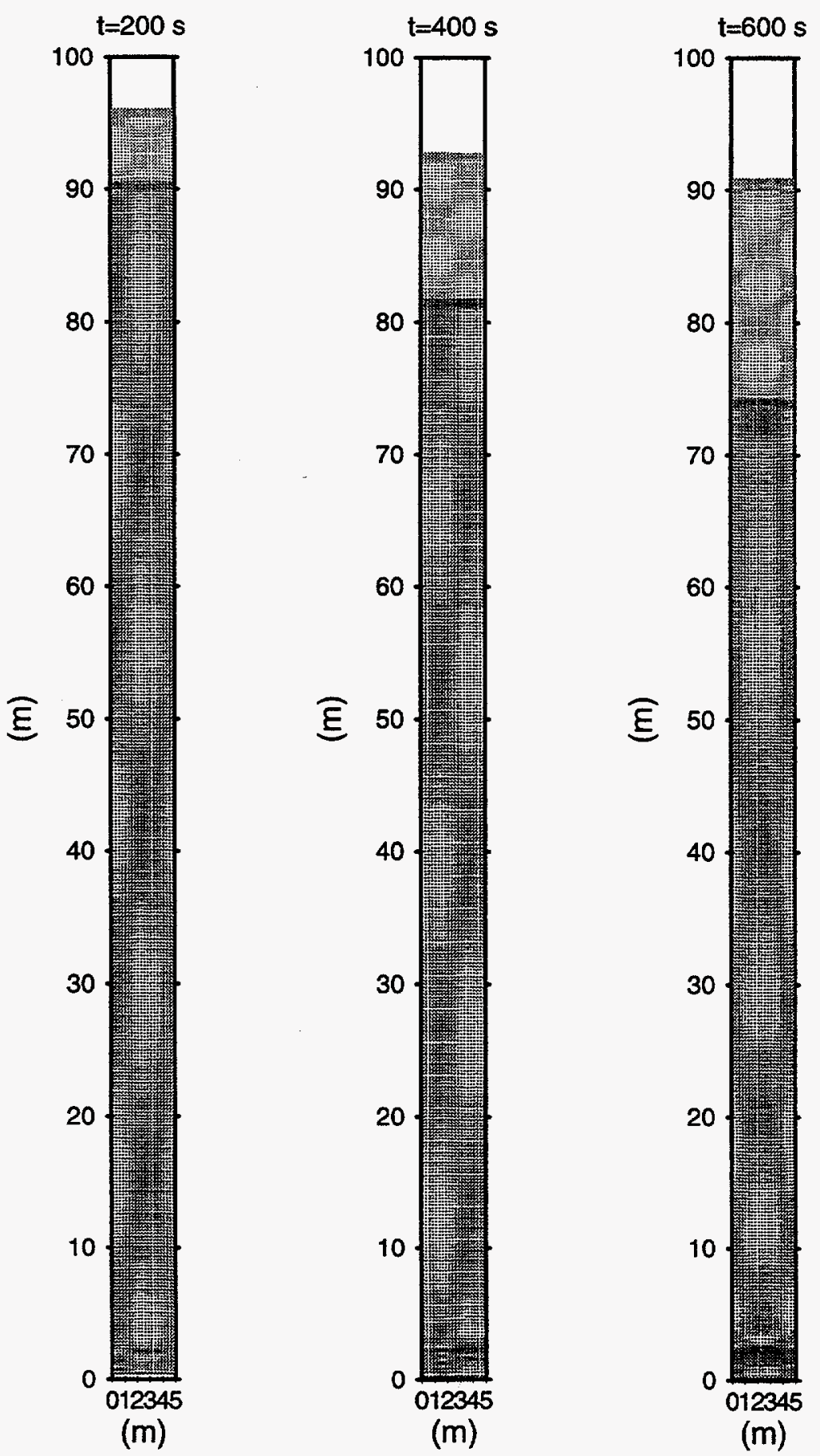

Figure 42. Particle distribution for batch sedimentation (Davis, et al., 1982). 
Figure 42 shows the particle distribution at three times. Three regions can be observed. The top region is fluid, the next region is light particles and fluid, and the lower region contains fluid, light particles and heavy particles. Particles concentrate in the lower section of the vessel and approach the close-pack limit. Figure 43 shows a one-dimensional and two-dimensional calculation of the interface levels compared with experimental data. The figure shows that the MP-PIC one-dimensional solution does a good job calculating the bimodal sedimentation. The MP-PIC two-dimensional solution does equally well in calculating the sedimentation rate. While the one-dimensional solution does an excellent job at considerable computational saving compared with the two-dimensional solution, it does not have the capability to calculate physical instabilities which may form in a suspension. Photographs by Weiland, et al. (1984) show the fingering structures produced by positive buoyancy particles. While the MP-PIC method has the potential for calculating these fingering structures, the calculations were not done in this study, in favor of simulating the inclined sedimentation.

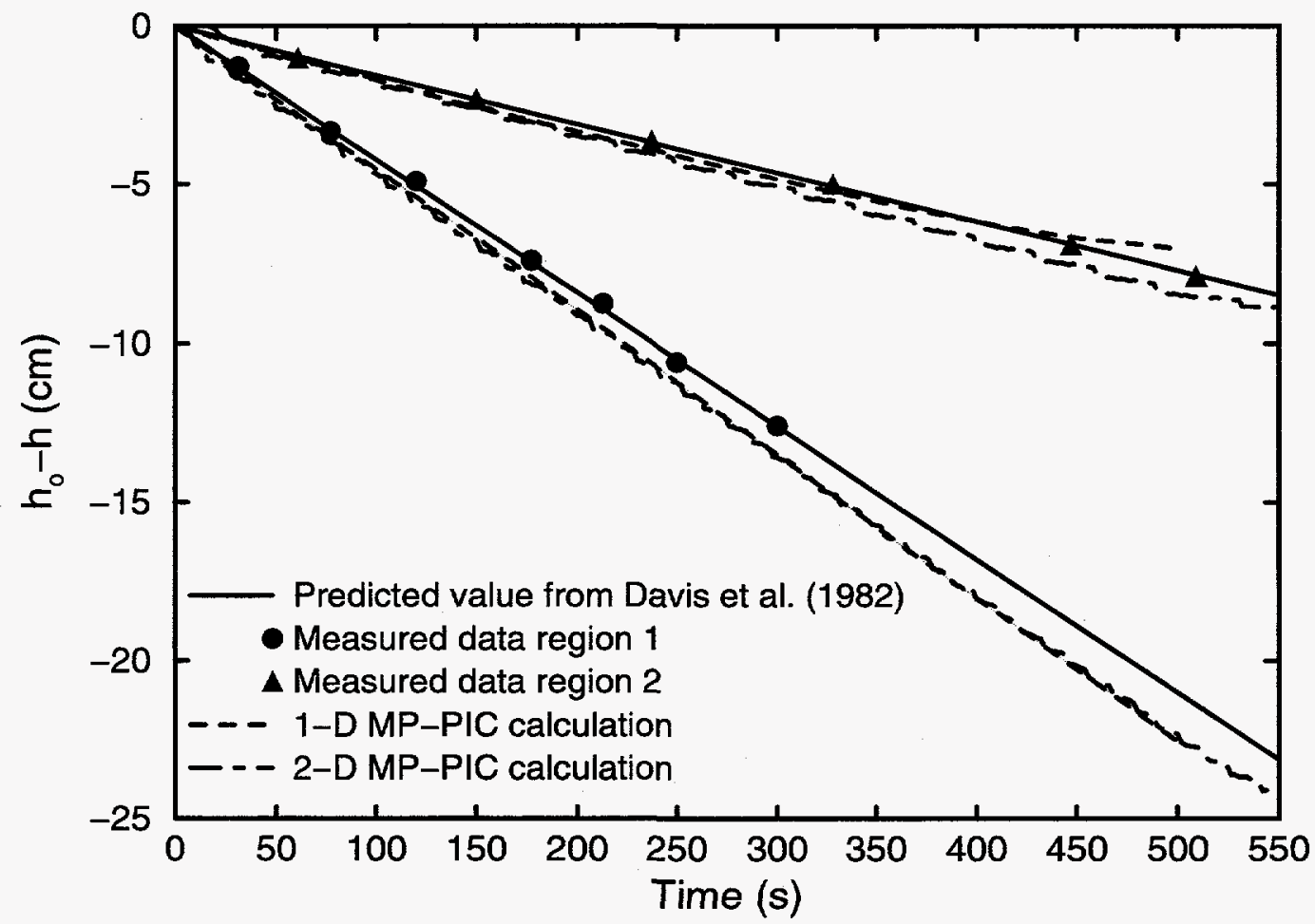

Figure 43. Sedimentation levels from bimodal suspension of particles (Davis, et al., 1982). 


\section{Inclined sedimentation}

The MP-PIC method is applied to the experiment given by Acrivos \& Herbolzheimer (1979). The container was $5 \mathrm{~cm}$ wide and $40 \mathrm{~cm}$ high, and experiments were run with the container tilted at $0^{\circ}, 20^{\circ}$, and $35^{\circ}$. The fluid density was $992 \mathrm{~kg} / \mathrm{m}^{3}$ and the viscosity was $0.0667 \mathrm{~kg} / \mathrm{m}-\mathrm{s}$. The particles were glass beads with a mean diameter of $137 \mu \mathrm{m}$ and density of $2420 \mathrm{~kg} / \mathrm{m}^{3}$. The MPPIC calculations for batch sedimentation used the same interphase drag coefficient as used in the other calculations in this study, and no attempt was made to tailor the calculation to batch sedimentation. All calculations were started with the container initially vertical (mixture-fluid interface was perpendicular to vessel walls) and then tilted incrementally over $5 \mathrm{~s}$ to the specified angle. The height of the mixture reported in this study, $h$, is illustrated below. Specifications for the MP-PIC calculation are given in Table 11 .

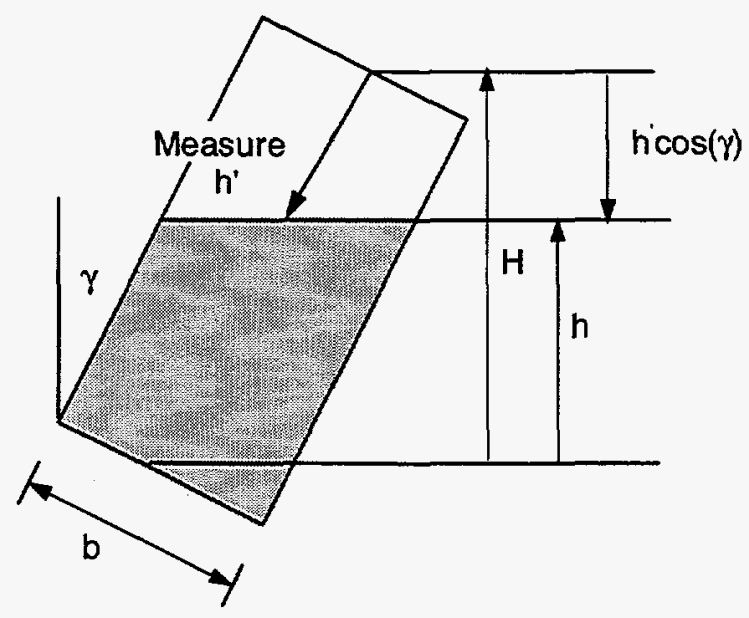

The MP-PIC calculated settling behavior of particles is the same as that observed in experiments and illustrated in Figure 41. The calculated particle distributions at $400 \mathrm{~s}$ for the container at $0^{\circ}, 20^{\circ}$ and $35^{\circ}$ are shown in Figure 44. Figure 45 shows the volume fraction of particles at times during the transient settling for the container tilted $35^{\circ}$.

The MP-PIC calculation captures the physics of the Boycott settling problem. Below the downward-facing surface, a thin layer of near clear-fluid forms above the suspension which is illustrated by $C$ in Figure 41. The thin layer is seen in the detail view shown in Figure 46. This wedge shaped layer is largest near the top, horizontal interface and decreases to zero in the lower part of the vessel where particles begin close-packing. On the opposite, upward-facing surface, particles concentrate forming a wedge which corresponds to $D$ in Figure 41 . The calculated wedge is small near the top interface and becomes larger moving down in the container. The central region illustrated as $B$ in Figure 41 and shown in Figure 46 is calculated to be homogenous from the top horizontal interface stretching down in the container to where particles begin to close pack. Figure 46 shows a strong concentration gradient below $10 \mathrm{~cm}$ where the particle bed is forming.

Figure 47 compares the measured and MP-PIC calculated transient settling rates for the container tilted $0^{\circ}, 20^{\circ}$, and $35^{\circ}$. The vertical sedimentation $\left(0^{\circ}\right)$ was predicted with a onedimensional and two-dimensional calculation, and both provide a good prediction of the vertical 
settling rate. Figure 47 shows that the two-dimensional MP-PIC calculations also compare well with measured inclined sedimentation data.

Table 11. Batch sedimentation. Container tilted $0^{\circ}, 20^{\circ}$ and $35^{\circ}$.

\begin{tabular}{lccc} 
Tilt & $0^{\circ}$ & $20^{\circ}$ & $35^{\circ}$ \\
Number of parcels & 8,000 & 10,000 & 10,000 \\
Initial particle volume fraction & 0.1 & 0.1 & 0.1 \\
Particle radius $(\mu \mathrm{m})$ & 68 & 68 & 68 \\
Particle density $\left(\mathrm{kg} / \mathrm{m}^{3}\right)$ & 2420 & 2420 & 2420 \\
Fluid density $\left(\mathrm{kg} / \mathrm{m}^{3}\right)$ & 992 & 992 & 992 \\
$\mathrm{P}_{\mathrm{s}}(\mathrm{Pa})$ & 75 & 75 & 75 \\
$\theta_{\mathrm{cp}}$ & 0.7 & 0.7 & 0.7 \\
$\mathrm{x}$-gravity, $\mathrm{y}$-gravity $\left(\mathrm{m} / \mathrm{s}^{2}\right)$ & $0,-9.8$ & $-3.35,-9.21$ & $-5.62,-8.027$ \\
Number x-cells & 5 & 15 & 15 \\
Number y-cells & 40 & 44 & 44 \\
x-domain $(\mathrm{cm})$ & 5 & 5 & 5 \\
y-domain $(\mathrm{cm})$ & 40 & 44 & 44 \\
Fluid viscosity $(\mathrm{kg} / \mathrm{m}-\mathrm{s})$ & 0.0667 & 0.0667 & 0.0667 \\
Time step $(\mathrm{s})$ & $0.5 \mathrm{~s} \mathrm{to} 650 \mathrm{~s}$ & 0.05 & 0.05 \\
& $0.05 \mathrm{~s}$ to $820 \mathrm{~s}$ & & \\
Max residual for $\theta_{\mathrm{p}}, \mathbf{u}_{\mathrm{g}}$ and $\delta \mathrm{p}$ & $10^{-8}$ & $10^{-8}$ & $10^{-8}$ \\
Relaxation on $\theta_{\mathrm{p}}, \mathbf{u}_{\mathrm{g}}$ and $\delta \mathrm{p}$ & $1,1,0.5$ & $1,1,0.5$ & $1,1,0.5$ \\
\hline \hline
\end{tabular}

The fluid and particle velocities for the tilted containers are shown in Figures 48 and 49 , respectively. A vortex pattern is calculated to form. The velocities are highest near the walls with fluid flowing upward under the downward-facing surface, and a dense mixture flowing down the upward-facing surface. A vortex pattern was observed in experiments by Kinosita (1949). Kinosita measured particle velocities as high as 100 times Stokes velocity. Hill, et al., calculated and measured particle velocities on the order of 10 times Stokes velocity. The MP-PIC calculated velocities compare favorably with measured velocities. The MP-PIC calculation produced maximum particle velocities on the order of 20 times Stokes velocity for the $20^{\circ}$ tilted container and 50 times Stokes velocity for the $35^{\circ}$ tilted container.

The calculated particle-mixture and fluid interface becomes wavy as seen from the figures. Particles entrained by rising fluid under the downward facing wall lift the upper section of the interface, and currents of downward flowing fluid and particles depress the central region of the interface. The entrainment of particles and the rounding of the corner at the horizontal surface has been experimentally observed and is apparent in the photographs by Herbolzheimer (1983) which are shown in Figure 50. The MP-PIC calculated interface shown in Figure 51 exhibits the same appearance as the interface experimentally observed by Herbolzheimer. The entrainment of particles by upward flowing fluid under top surface is more pronounced for the $20^{\circ}$ tilted vessel than the $35^{\circ}$ tilted vessel. As time progresses and particles settle out, the entrainment decreases, and the mixture-fluid interface becomes flatter (Figure 45). 

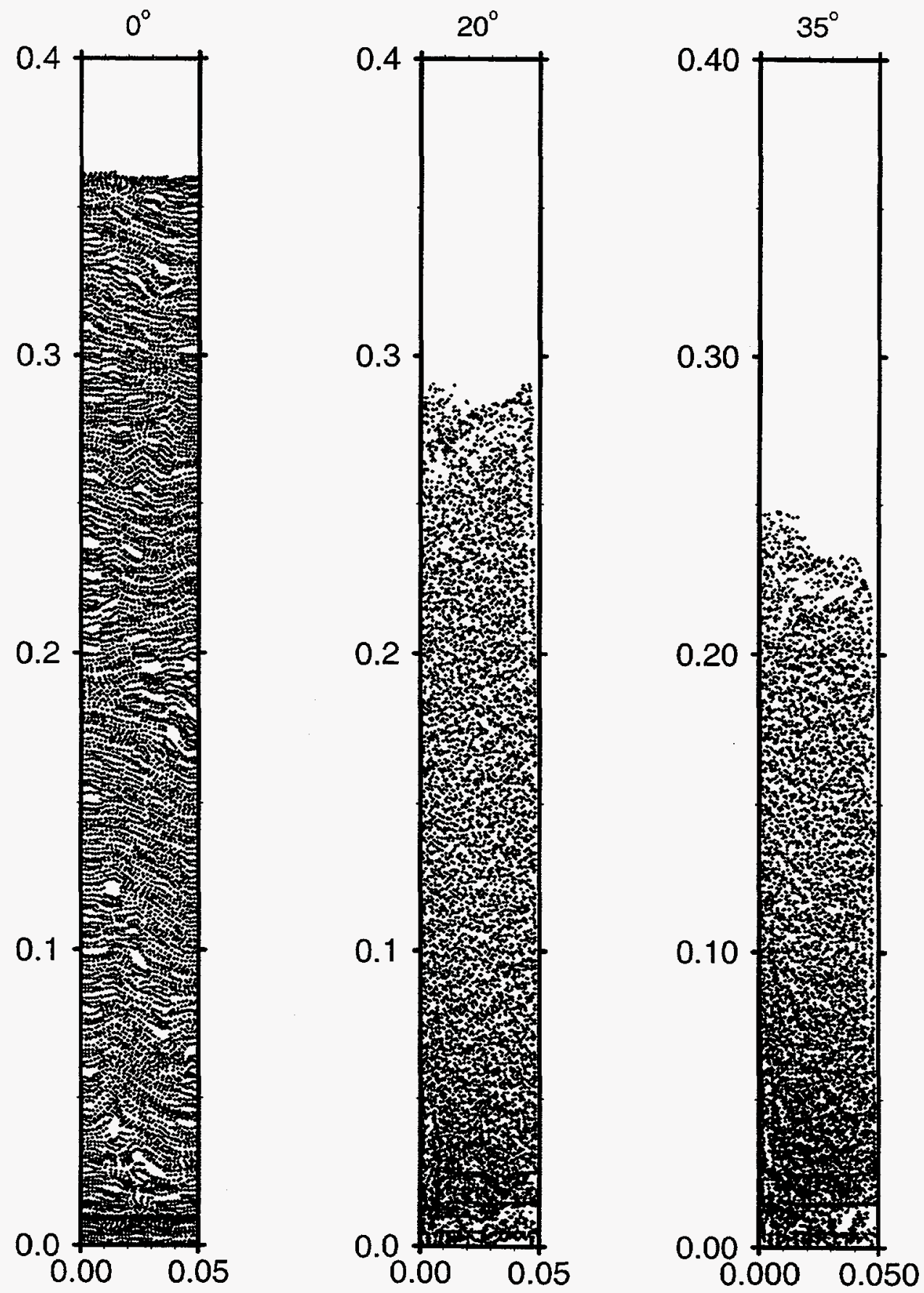

Figure 44. The particle distributions for batch sedimentation at $400 \mathrm{~s}$ with the container inclined $0^{\circ}, 20^{\circ}$, and $35^{\circ}$. 

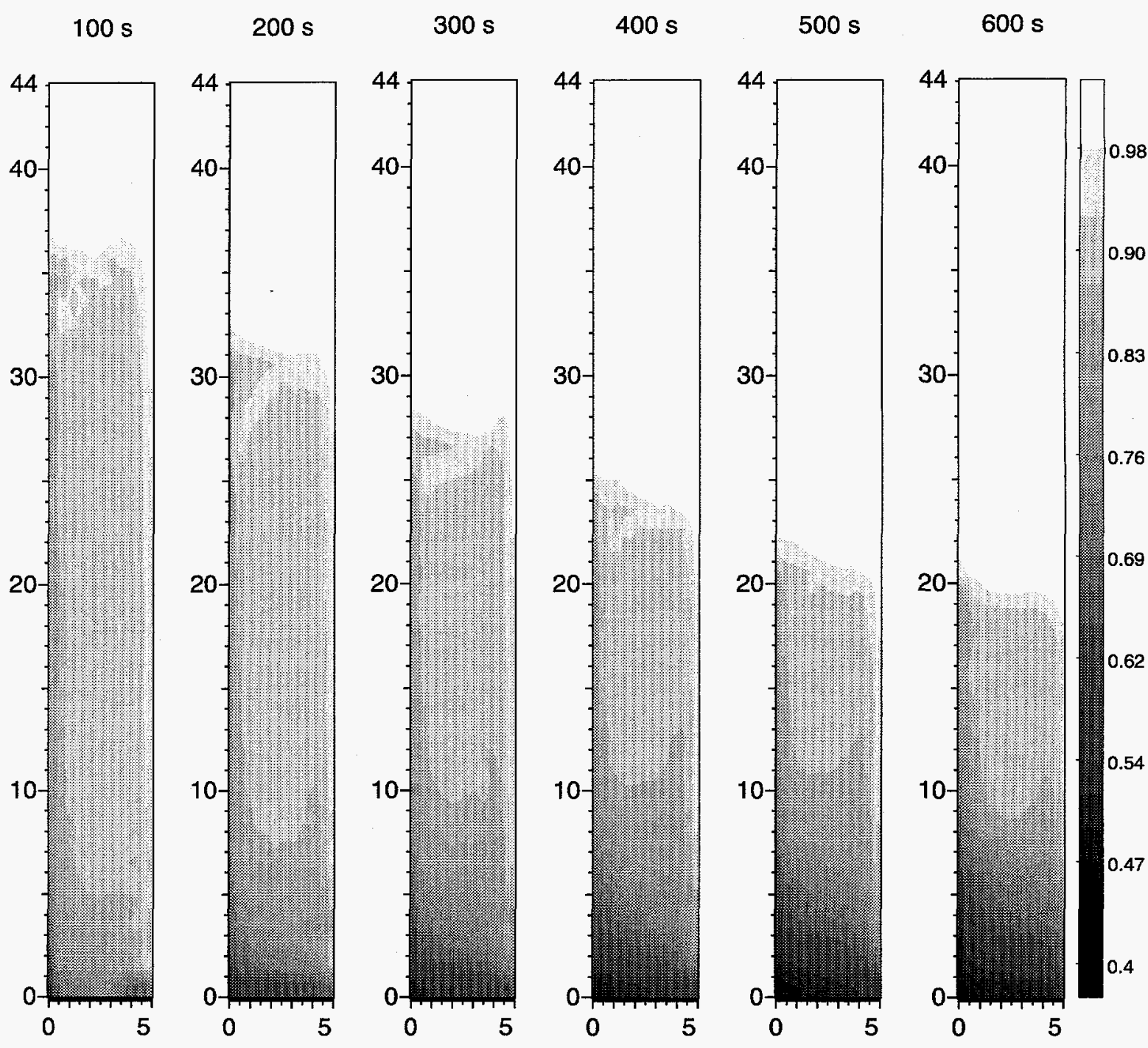

Figure 45. Batch sedimentation. Container tilted $35^{\circ}$. 


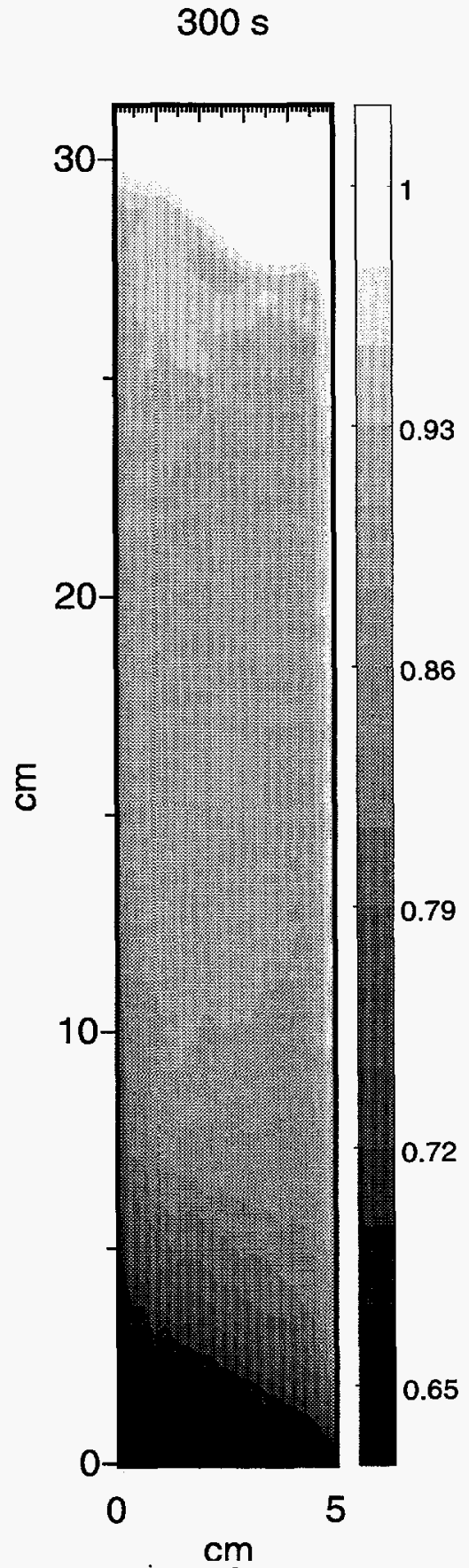

Figure 46. Batch sedimentation. Container tilted $35^{\circ}$ and $t=300$ s. MP-PIC calculation with $30 \mathrm{x}-$ nodes, $88 \mathrm{y}$-nodes and 15,000 particles. 


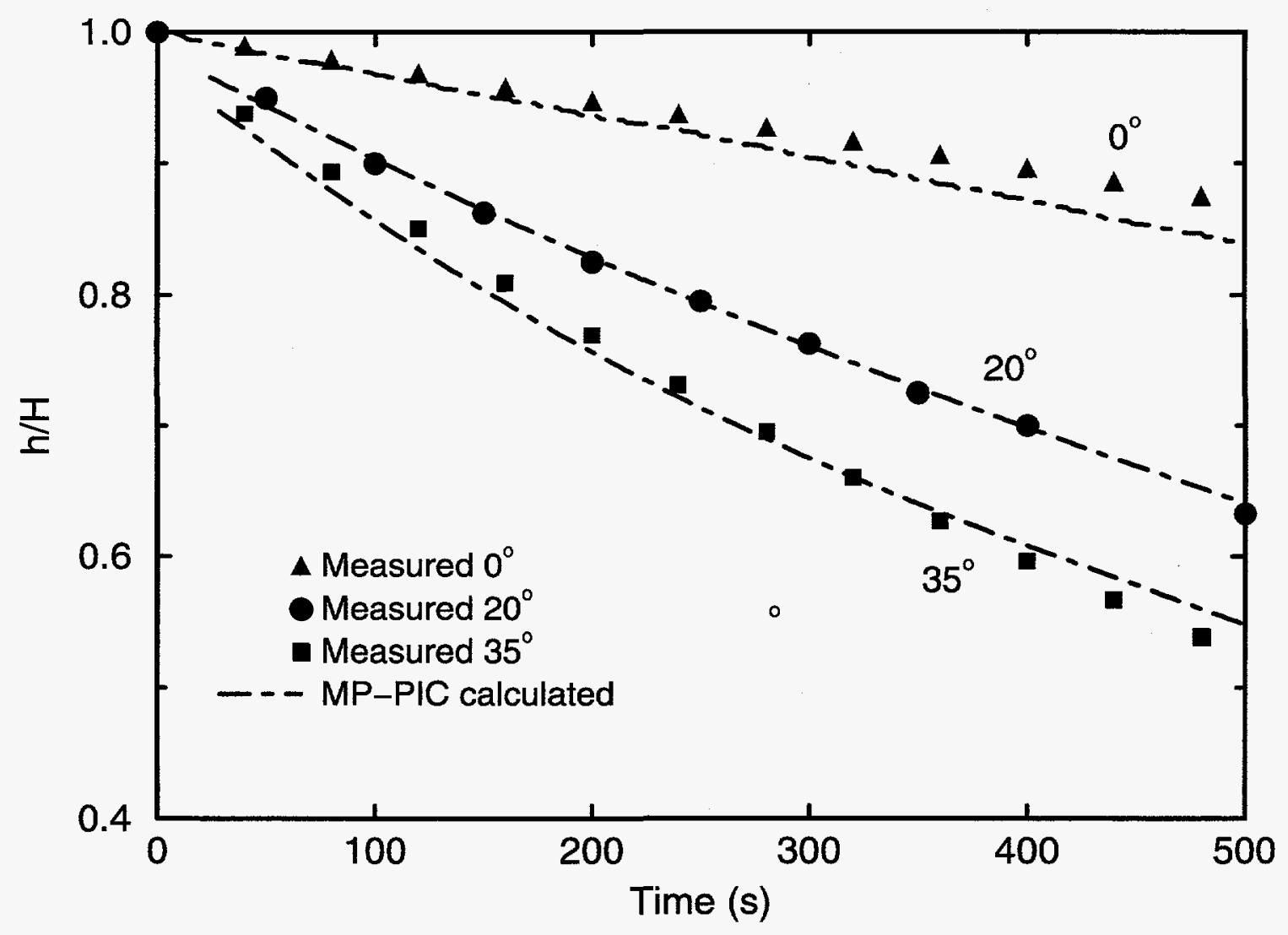

Figure 47. MP-PIC calculated and measured interface for suspension of particles (Acrivos \& Herbolzheimer 1979). 


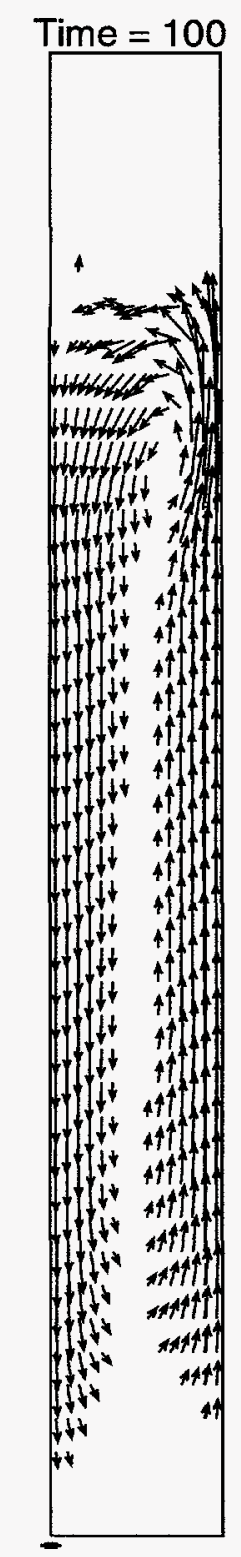

$0.1 \mathrm{~cm} / \mathrm{s}$

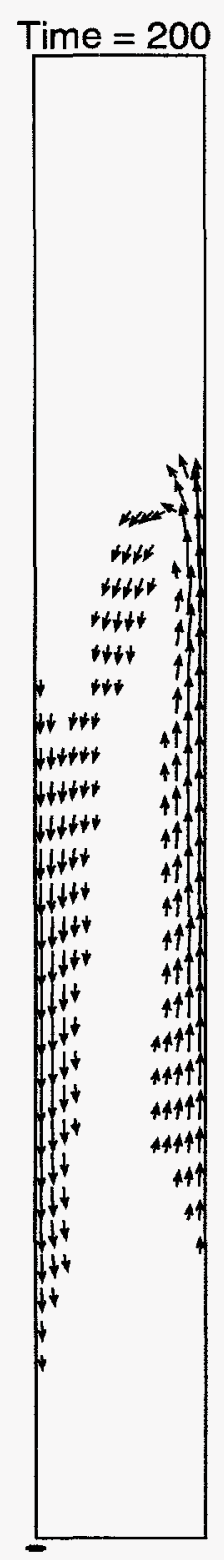

$0.1 \mathrm{~cm} / \mathrm{s}$

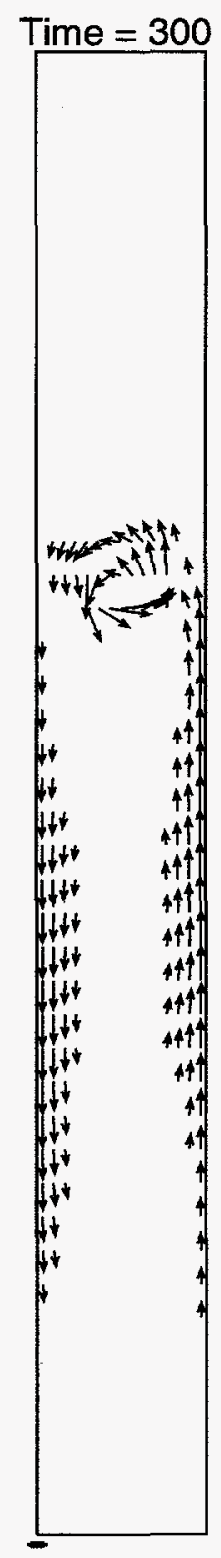

$0.1 \mathrm{~cm} / \mathrm{s}$

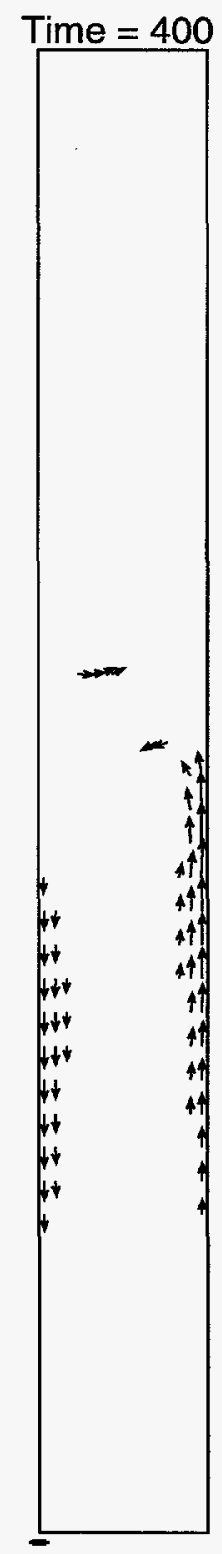

$0.1 \mathrm{~cm} / \mathrm{s}$

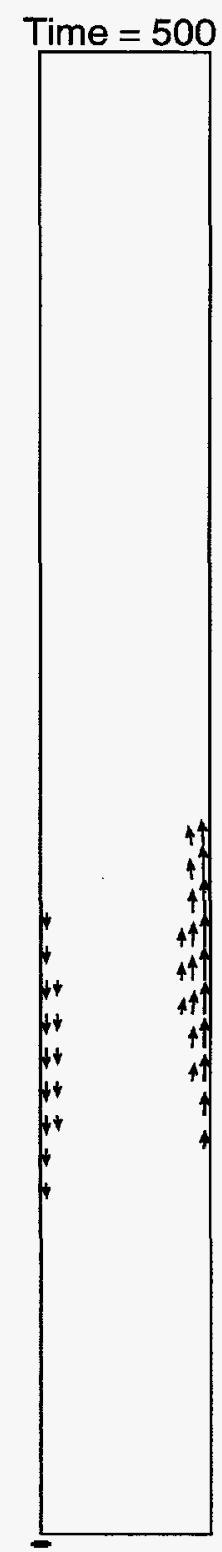

$0.1 \mathrm{~cm} / \mathrm{s}$
Time $=600$

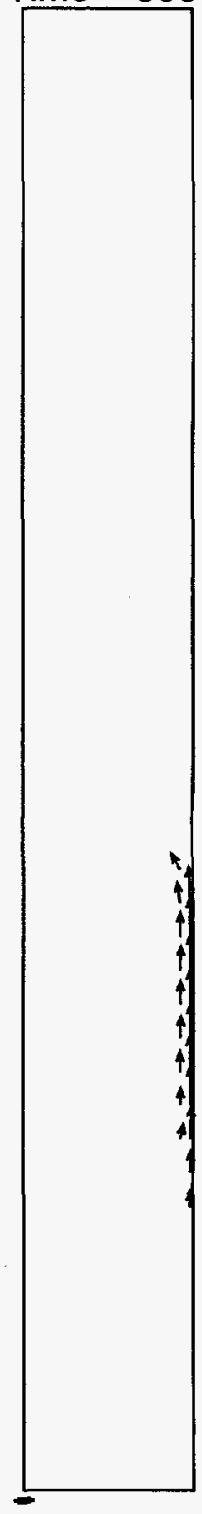

$0.1 \mathrm{~cm} / \mathrm{s}$

Figure 48. Fluid velocity for container tilted $35^{\circ}$. 


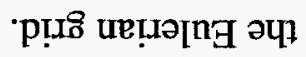

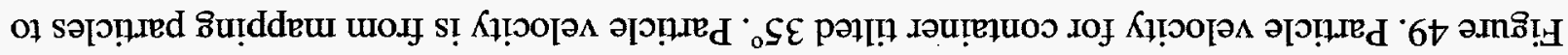

S/40 L.0

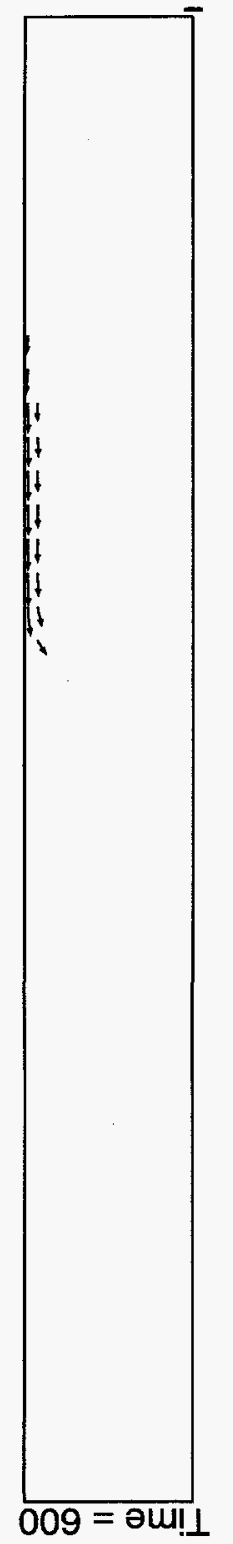

S/uo 1.0

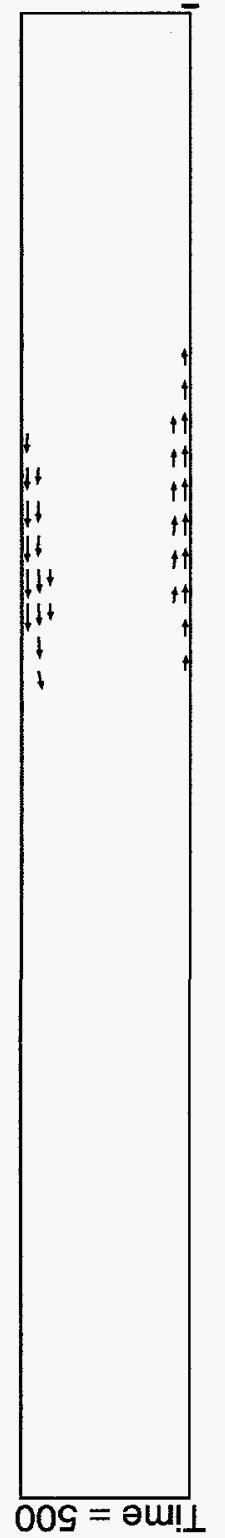

s/uo म 0

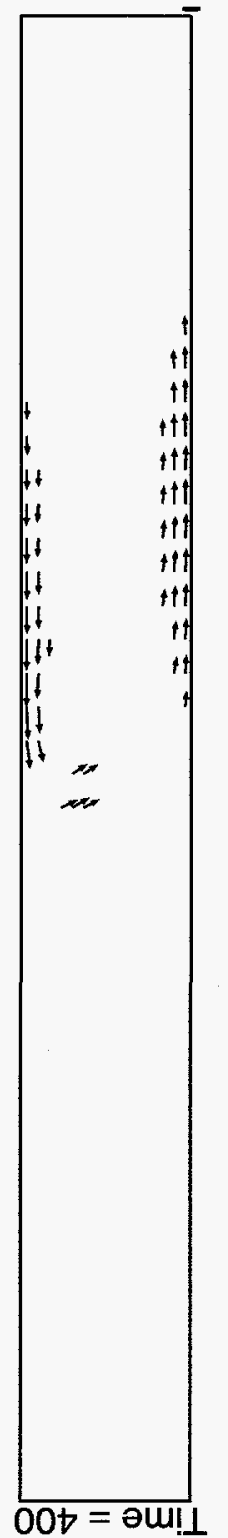

s/uo 1.0

s/uo 1.0

s/uo L'O
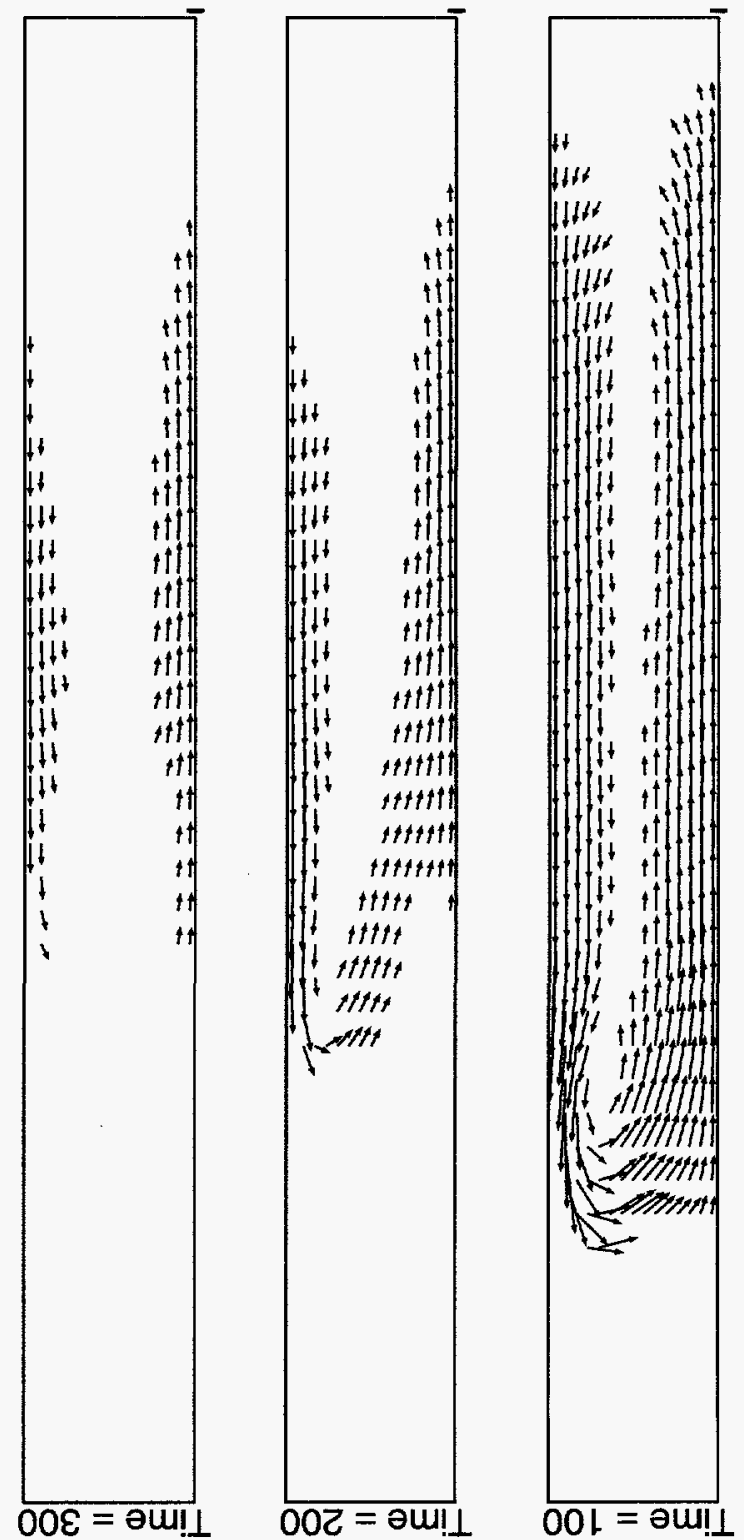
(a)

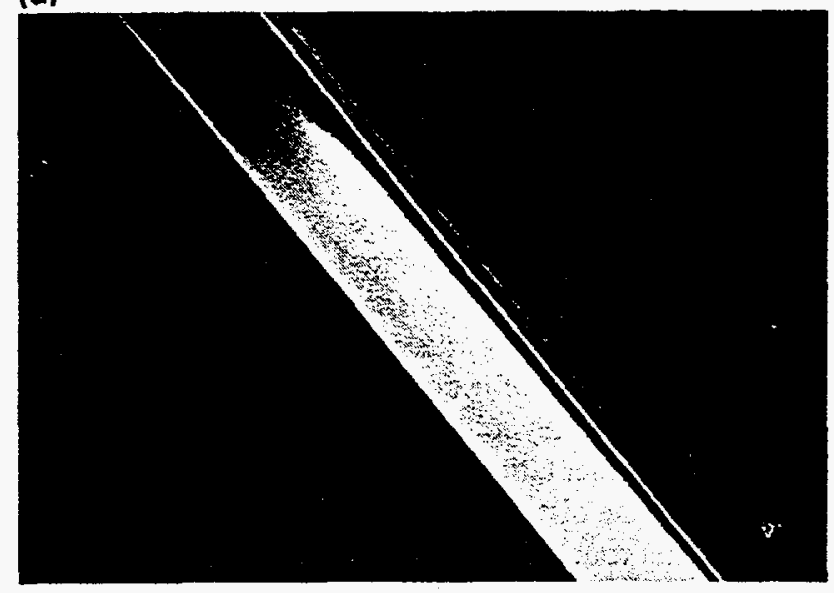

(b)

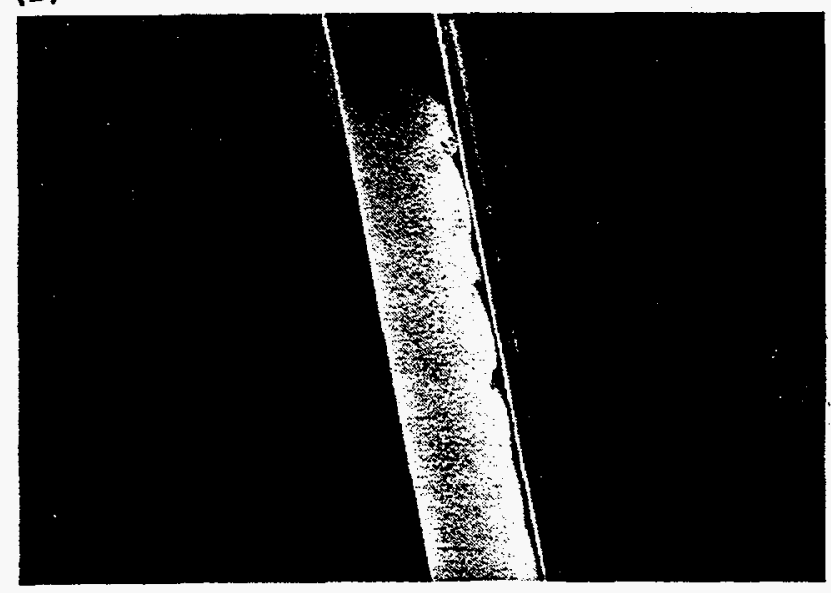

Figure 50. Observation of the flow for a volume fraction of 0.01 and fluid viscosity of $18.8 \mathrm{cP}$. (a) angle of inclination large; (b) angle is decreased and waves appear along interface in the upper region of channel. Reprinted with permission from Herbolzheimer, E., 1983, "Stability of the flow during sedimentation in inclined channels," Phys. Fluids, vol. 26, Fig. 2, p. 2044. Copyright 1983 American Institute of Physics.

\section{E. Grid independence}

Grid independence was checked by calculating batch inclined sedimentation using double the number of $\mathrm{x}$ and $\mathrm{y}$ nodes ( $15 \mathrm{x} 44$ nodes to $30 \times 88$ nodes). The number of particle clouds was increased from 10,000 to 15,000 . The time step was reduced to $0.01 \mathrm{~s}$. The other specifications are given in Table 10 for the container tilted $35^{\circ}$. The particle distribution is shown in Figure 51. The mixture-fluid interface is smoother for the finer grid. The finer grid spacing also has a more uniform particle distribution at the bottom of the vessel where high particle stress gradients form as particles close pack. Both the calculated behavior and the sedimentation rate are the same for both the fine and the coarse grid spacing. 


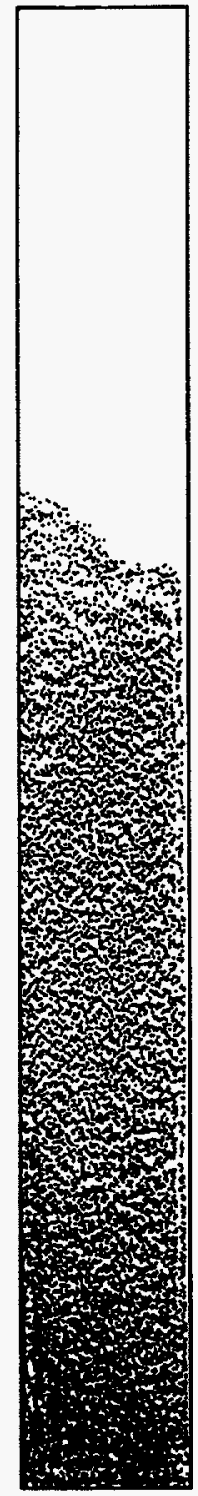

Figure 51. Batch sedimentation. Fine grid spacing. Particle distribution at $300 \mathrm{~s}$ for container tilted $35^{\circ}$. 


\section{CONCLUDING REMARKS}

A two-dimensional, incompressible MP-PIC method has been developed to obtain accurate numerical simulations for a variety of fluid and particle flows. Rayleigh-Taylor and sedimentation problems showed the two-dimensional MP-PIC method can calculate mixture flows from dilute particles to close-pack particle beds. Fluidized bed problems showed the MP-PIC method can calculate the highly dynamic mixing of sparse and dense particle flow.

All the examples presented in this study used the same modeling and the same constituent relations, such as particle-fluid interface drag coefficient (see Equations 7-11). The comparison of MP-PIC calculated data with experimental data for the variety of problems was good, with none of the calculations being tuned to the problem being solved.

The one modeling exception was the need to use an explicit particle velocity in the continuum interphase momentum transfer for batch sedimentation. Using the implicit particle velocity produced instabilities which led to large non-physical circulating flows. The stability of the MP-PIC method with the current physical modeling needs further investigation. 


\section{REFERENCES}

Acrivos, A. and Herbolzheimer, E., 1979, "Enhanced sedimentation in settling tanks with inclined walls," J. Fluid Mech., vol. 92, pp.435-457.

Al-Naafa', M. A. and Selim, M. S., 1989, "Sedimentation of polydisperse concentrated suspensions," The Canadian Journal of Chemical Engineering, vol. 67, pp. 253-263.

Amsden, A. A., O'Rourke, P. J., and Butler, T. D., 1989, "KIVA-II: A computer program for chemically reactive flows with sprays," LA-11560-MS, Los Alamos National Lab.

Andrews, M. J. and O'Rourke, P. J., 1996, "The multiphase particle-in-cell (MP-PIC) method for dense particle flow," Int. J. Multiphase Flow, vol. 22, pp. 379-402.

Auzerais, F. M., Jackson, R., and Russel, W. B., 1988, "The resolution of shocks and the effects of compressible sediments in transient settling," J. Fluid Mech., vol. 195, pp. 437462.

Batchelor, G. K., 1988, "A new theory of the instability of a uniform fluidized bed," J. Fluid Mech., vol. 193, pp. 75-110.

Birkoff, G, 1960, "Helmholtz and Taylor instability," in Proceedings of the XIII Applied Mathematics Symposium, American Mathematical Society, Providence, RI.

Boycott, A. E., 1920, "Sedimentation of blood corpuscles," Nature, vol. 104, p. 532.

Chandrasekhar, S., 1961, Hydrodynamic and Hydromagnetic Stability, Oxford University Press.

Chao, B. T., 1996, Personal conversation regarding experiments reported by Lin, J. S., Chen, M. M., and Chao, B. T., 1985.

Daly, B., 1967, "Numerical study of two fluid Rayleigh-Taylor instability," Phys. Fluids, vol. 10, p. 297.

Davis, R. H., Herbolzheimer, E., and Acrivos, A., 1985, "Sedimentation of noncolloidal particles at low Reynolds numbers," Annul. Rev. Fluid Mech., vol. 17, pp. 91-118.

Davis, R. H., Herbolzheimer, E., and Acrivos, A., 1982, "The sedimentation of polydisperse suspensions in vessels having inclined walls," Int. J. Multiphase Flow, vol. 8, no. 6, pp. 571-585.

Ding, J. and Gidaspow, D.,1990, "A bubbling fluidization model using kinetic theory of granular flow," AIChE Journal, vol. 36, pp. 523-538. 
Gidaspow, D., 1986, "Hydrodynamics of fluidization and heat transfer: supercomputer modeling," Appl. Mech. Rev., vol. 39, pp. 1-22.

Gidaspow, D., 1994, Multiphase flow and fluidization continuum and kinetic theory description, Academic Press, Boston.

Harlow, F. H. and Amsden, A. A., 1971, "Fluid dynamics, A LASL Monograph," LA-4700.

Harris, S. E. and Crighton, D. G., 1994, "Solitons, solitary waves and voidage disturbances in gas-fluidized beds," J. Fluid Mech., vol. 266, pp. 243-276.

Hill, W. D., Tothfus, R. R., and Li, K., 1977, "Boundary-enhanced sedimentation due to settling convection," Int. J. Multiphase Flow, vol. 3, pp. 561-583.

Kapoor, B. and Acrivos, A., 1995, "Sedimentation and sediment flow in settling tanks with inclined walls," J. Fluid Mech., vol. 290, pp. 39-66.

Kinosita, K., 1949, "Sedimentation in tilted vessels," J. Colloid Interface Sci., vol. 4, pp. 166-176.

Lewis, D. J., 1950, "The instability of liquid surfaces when accelerated in a direction perpendicular to their plane, II," Proc. Royal Soc., 202A, p 81.

Lin, J. S., Chen, M. M., and Chao, B. T., 1985, "A novel radioactive particle tracking facility for measurement of solid motion in gas fluidized beds," AIChE J., vol. 31, pp. 465473.

Linden, P. F., Redondo, J. M., and Youngs, L., 1994, "Molecular mixing in Rayleigh-Taylor instability," J. Fluid Mech., vol. 267, pp. 97-124.

Mirza, S. and Richardson, J. F., 1978, "Sedimentation of suspensions of particles of two or more sizes," Chem. Eng. Sci., vol. 34, pp. 447-454.

Nakumura, H., and Kuroda, K., 1937, "La cause de l'acceleration de la vitesse de sedimentation des suspension dans les recipents inclines," Keijo. Med., vol. 8, pp. 191-195.

O'Rourke, P. J., 1981, Collective drop effects on vaporizing liquid sprays, Ph.D. Thesis, Princeton University.

O'Rourke, P. J. and Amsden, A. A., 1986, "Implementation of a conjugate residual iteration in the KIVA computer program," Los Alamos Report LA-10849-MS, Los Alamos National Laboratory, Los Alamos, NM.

Patankar, S. V., 1980, Numerical heat transfer and fluid flow, Hemisphere Publishing Corporation, New York, NY.

Ponder, E., 1925, "On sedimentation and rouleaux formation," Quart J. Expt. Physol., vol. 15 , pp. 235-252. 
Ratafia, M., 1973, "Experimental investigation of Taylor instability," Phys. Fluids, vol. 16, pp. 1207-1210.

Risk, M. A., 1993, "Mathematical modeling of densely loaded, particle laden turbulent flows," Atomization and Sprays, vol. 3, pp. 1-27.

Shih, Y. T., Gidaspow, D. and Wasan, D. T., 1987, "Hydrodynamics of sedimentation of multisized particles," Powder Technology, vol. 50, pp. 201-215.

Spalding, D. B., 1972, "A novel finite-difference formulation for differential expressions involving both first and second derivatives," International Journal for Numerical Methods Engineering, 4, p. 551.

Taylor, G. I., 1950, "The instability of liquid surfaces when accelerated in a direction perpendicular to their planes I," Proc. Royal Soc. London, Ser. A, vol. 201, p 192.

Weiland, R. H., Fessas, Y. P. and Ramaro, B. V., 1984, "On stabilities arising during sedimentation of two-component mixtures of solids," J. Fluid Mech., vol. 142, pp. 383389.

Werther, J. and Molerus, O., 1973, "The local structure of gas fluidized beds. II: The spatial distribution of bubbles," Int. J. Multiphase Flow, vol. 1, p.123.

Williams, F. A., 1985, Combustion theory, 2nd edition, Benjamin/Cummings Publishing Company, Menlo Park, CA.

Youngs, D. L., 1984, "Numerical simulation of turbulent mixing by Rayleigh-Taylor instability," Physica 12D, p 32.

Youngs, D. L., 1989, "Modeling turbulent mixing by Rayleigh-Taylor instabilities," Physica $37 D$, pp. 270-287. 


\section{APPENDIX}

\section{A. Momentum equation coefficients}

The u-momentum equation is aproximated by linear algabraic equations with independent variables u-velocity, pressure and particle volume fraction. The $u$-velocity at face node $(i+1 / 2, j)$ is given by equation (46)

$$
\begin{gathered}
c_{u_{c c}}\left(u_{g}\right)_{i+1 / 2, j}^{n+1}=C_{u_{i+1 / 2, j}} \\
+c_{u_{r r}}\left(u_{g}\right)_{i+3 / 2, j}^{n+1}+c_{u_{l} l}\left(u_{g}\right)_{i-1 / 2, j}^{n+1}+c_{u_{t}}\left(u_{g}\right)_{i+1 / 2, j+1}^{n+1}+c_{u_{b b}}\left(u_{g}\right)_{i+1 / 2, j-1}^{n+1} \\
+c_{u_{t r}}\left(u_{g}\right)_{i+3 / 2, j+1}^{n+1}+c_{u_{l l}}\left(u_{g}\right)_{i-1 / 2, j+1}^{n+1}+c_{u_{b r}}\left(u_{g}\right)_{i+3 / 2, j-1}^{n+1}+c_{u b l}\left(u_{g}\right)_{i-1 / 2, j-1}^{n+1} \\
+a_{u l l}(p)_{i, j}^{n+1}+a_{u_{r r}}(p)_{i+1, j}^{n+1}+a_{u b l}(p)_{i, j-1}^{n+1}+a_{u_{b r}}(p)_{i+1, j-1}^{n+1}+a_{u_{t l}}(p)_{i, j+1}^{n+1}+a_{u_{t r}}(p)_{i+1, j+1}^{n+1} \\
+b_{u l l}\left(\theta_{p}\right)_{i, j}^{n+1}+b_{u_{r r}}\left(\theta_{p}\right)_{i+1, j}^{n+1}+b_{u_{b l}}\left(\theta_{p}\right)_{i, j-1}^{n+1}+b_{u_{b r}}\left(\theta_{p}\right)_{i+1, j-1}^{n+1}+b_{u_{t l}}\left(\theta_{p}\right)_{i, j+1}^{n+1}+b_{u_{t r}}\left(\theta_{p}\right)_{i+1, j+1}^{n+1} .
\end{gathered}
$$

The nodilization for the u-velocity is shown below.

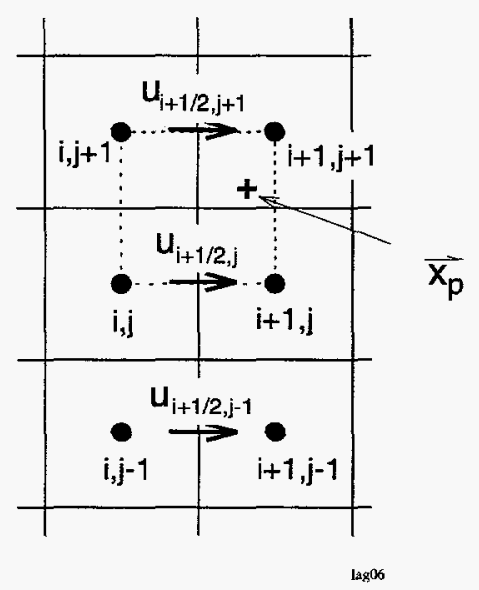

Coefficients on velocity arise from time rate of change of momentum, convection and interphase drag. The coefficients on the $u$-velocity and its neighbors are

$$
c_{u_{l l}}=D_{i, j}+\max \left(0, f_{i, j}\right)+\sum_{p} \frac{m_{p} N_{p} T\left(\mathbf{x}_{p}\right)_{i+1 / 2, j} D_{p}}{\left(1+D_{p} \Delta t\right)} S\left(\mathbf{x}_{p}\right)_{i-1 / 2, j}
$$




$$
\begin{aligned}
& c_{u_{r r}}=D_{i+1, j}+\max \left(0,-f_{i+1, j}\right)+\sum_{p} \frac{m_{p} N_{p} T\left(\mathbf{x}_{p}\right)_{i+1 / 2, j} D_{p}}{\left(1+D_{p} \Delta t\right)} S\left(\mathbf{x}_{p}\right)_{i+3 / 2, j} \\
& c_{u_{b b}}=D_{i+1 / 2, j-1 / 2}+\max \left(0, f_{i+1 / 2, j-1 / 2}\right)+\sum_{p} \frac{m_{p} N_{p} T\left(\mathbf{x}_{p}\right)_{i+1 / 2, j} D_{p}}{\left(1+D_{p} \Delta t\right)} S\left(\mathbf{x}_{p}\right)_{i+1 / 2, j-1} \\
& c_{u_{t t}}=D_{i+1 / 2, j+1 / 2}+\max \left(0,-f_{i+1 / 2, j+1 / 2}\right)+\sum_{p} \frac{m_{p} N_{p} T\left(\mathbf{x}_{p}\right)_{i+1 / 2, j} D_{p}}{\left(1+D_{p} \Delta t\right)} S\left(\mathbf{x}_{p}\right)_{i+1 / 2, j+1} \\
& c_{u_{t r}}=\sum_{p} \frac{m_{p} N_{p} T\left(\mathbf{x}_{p}\right)_{i+1 / 2, j} D_{p}}{\left(1+D_{p} \Delta t\right)} S\left(\mathbf{x}_{p}\right)_{i+3 / 2, j+1} \\
& c_{u_{t l}}=\sum_{p} \frac{m_{p} N_{p} T\left(\mathbf{x}_{p}\right)_{i+1 / 2, j} D_{p}}{\left(1+D_{p} \Delta t\right)} S\left(\mathbf{x}_{p}\right)_{i-1 / 2, j+1} \\
& c_{u_{b r}}=\sum_{p} \frac{m_{p} N_{p} T\left(\mathbf{x}_{p}\right)_{i+1 / 2, j} D_{p}}{\left(1+D_{p} \Delta t\right)} S\left(\mathbf{x}_{p}\right)_{i+3 / 2, j-1} \\
& c_{u b l}=\sum_{p} \frac{m_{p} N_{p} T\left(\mathbf{x}_{p}\right)_{i+1 / 2, j} D_{p}}{\left(1+D_{p} \Delta t\right)} S\left(\mathbf{x}_{p}\right)_{i-1 / 2, j-1} \\
& c_{u_{c c}}=D_{i, j}+\max \left(0,-f_{i, j}\right)+D_{i+1, j}+\max \left(0, f_{i+1, j}\right) \\
& +D_{i+1 / 2, j-1 / 2}+\max \left(0,-f_{i+1 / 2, j-1 / 2}\right)+D_{i+1 / 2, j+1 / 2}+\max \left(0, f_{i+1 / 2, j+1 / 2}\right) \\
& +\frac{\rho_{g} V_{i+1 / 2, j}}{\Delta t}+\sum_{p} \frac{m_{p} N_{p} T\left(\mathbf{x}_{p}\right)_{i+1 / 2, j} D_{p}}{\left(1+D_{p} \Delta t\right)} S\left(\mathbf{x}_{p}\right)_{i+1 / 2, j}
\end{aligned}
$$

The max function specifies the maximum value of the two arguments. The $\Delta t$ is the time interval, $N_{p}$ is the number of particles in a parcel, $m_{p}$ is the mass of a particle in the parcel, and $D_{p}$ is the drag coefficient for a particle in the parcel. The mass flux and diffusion coefficients for Cartesian coordinates are

$$
f_{i, j}=\rho_{g}\left(\theta_{g} u_{g}\right)_{i, j}^{n} \Delta y_{j} \quad D_{i, j}=\frac{\left(\theta_{g} \mu_{g}\right)_{i, j}^{n} \Delta y_{j}}{\delta x}
$$




$$
\begin{array}{ll}
f_{i+1, j}=\rho_{g}\left(\theta_{g} u_{g}\right)_{i+, j}^{n} \Delta y_{j} & D_{i+1, j}=\frac{\left(\theta_{g} \mu_{g}\right)_{i+1, j}^{n} \Delta y_{j}}{\delta x} \\
f_{i+1 / 2, j-1 / 2}=\rho_{g}\left(\theta_{g} v_{g}\right)_{i+1 / 2, j-1 / 2}^{n} \delta x_{i} & D_{i+1 / 2, j-1 / 2}=\frac{\left(\theta_{g} \mu_{g}\right)_{i+1 / 2, j-1 / 2}^{n} \Delta x_{i}}{\delta y} \\
f_{i+1 / 2, j+1 / 2}=\rho_{g}\left(\theta_{g} v_{g}\right)_{i+1 / 2, j-1 / 2}^{n} \delta x_{i} & D_{i+1 / 2, j+1 / 2}=\frac{\left(\theta_{g} \mu_{g}\right)_{i+1 / 2, j+1 / 2}^{n} \Delta x_{i}}{\delta y}
\end{array}
$$

where $\theta_{g}$ is gas volume fraction, $\mu_{g}$ is gas viscosity and $\rho_{g}$ is the gas density. The cell center velocities, $u$ and $v$, in the flux terms are taken as the average of the adjacent face center velocities, e.g.,

$$
\left(\theta_{g} u_{g}\right)_{i, j}^{n}=\frac{\left(\theta_{g} u_{g}\right)_{i-1 / 2, j}^{n}+\left(\theta_{g} u_{g}\right)_{i+1 / 2, j}^{n}}{2}
$$

and similarly for other cell center velocities.

The pressure coefficients in Cartesian coordinates are

$$
\begin{aligned}
a_{u_{l l}}=\Delta y_{j}+\sum_{p} \frac{m_{p} N_{p} T\left(\mathbf{x}_{p}\right)_{i+1 / 2, j}}{\left(1+D_{p} \Delta t\right) \rho_{p}}\left[\nabla S\left(\mathbf{x}_{p}\right)_{i, j} \cdot \mathbf{e}_{x}\right] \\
a_{u_{r r}}=-\Delta y+\sum_{p} \frac{m_{p} N_{p} T\left(\mathbf{x}_{p}\right)_{i+1 / 2, j}}{\left(1+D_{p} \Delta t\right) \rho_{p}}\left[\nabla S\left(\mathbf{x}_{p}\right)_{i+1, j} \cdot \mathbf{e}_{x}\right] \\
a_{u_{t r}}=\sum_{p} \frac{m_{p} N_{p} T\left(\mathbf{x}_{p}\right)_{i+1 / 2, j}}{\left(1+D_{p} \Delta t\right) \rho_{p}}\left[\nabla S\left(\mathbf{x}_{p}\right)_{i+1, j+1} \cdot \mathbf{e}_{x}\right] \\
a_{u_{t l}}=\sum_{p} \frac{m_{p} N_{p} T\left(\mathbf{x}_{p}\right)_{i+1 / 2, j}}{\left(1+D_{p} \Delta t\right) \rho_{p}}\left[\nabla S\left(\mathbf{x}_{p}\right)_{i, j+1} \cdot \mathbf{e}_{x}\right] \\
a_{u_{b l}}=\sum_{p} \frac{m_{p} N_{p} T\left(\mathbf{x}_{p}\right)_{i+1 / 2, j}}{\left(1+D_{p} \Delta t\right) \rho_{p}}\left[\nabla S\left(\mathbf{x}_{p}\right)_{i, j-1} \cdot \mathbf{e}_{x}\right] \\
a_{u_{b r}}=\sum_{p} \frac{m_{p} N_{p} T\left(\mathbf{x}_{p}\right)_{i+1 / 2, j}}{\left(1+D_{p} \Delta t\right)_{p}}\left[\nabla S\left(\mathbf{x}_{p}\right)_{i+1, j-1} \cdot \mathbf{e}_{x}\right]
\end{aligned}
$$


The particle volume fraction is introduced in the u-momentum equation through the interparticle stress in the interphase drag. The coefficients on particle volume fraction are

$$
\begin{aligned}
& b_{u_{l l}}=-\sum_{p} \frac{m_{p} N_{p} T\left(\mathbf{x}_{p}\right)_{i+1 / 2, j} \Delta t D_{p}}{\left(1+D_{p} \Delta t\right) \rho_{p} \theta_{p_{i+1 / 2, j}}^{n}}\left[\nabla S\left(\mathbf{x}_{p}\right)_{i, j} \cdot \mathbf{e}_{x}\right] C_{1 p_{\tau}} \\
& b_{u_{r r}}=-\sum_{p} \frac{m_{p} N_{p} T\left(\mathbf{x}_{p}\right)_{i+1 / 2, j} \Delta t D_{p}}{\left(1+D_{p} \Delta t\right) \rho_{p} \theta_{p_{i+1 / 2, j}^{n}}^{n}}\left[\nabla S\left(\mathbf{x}_{p}\right)_{i+1, j} \cdot \mathbf{e}_{x}\right] C_{p_{p_{\tau}}} \\
& b_{u_{t r}}=-\sum_{p} \frac{m_{p} N_{p} T\left(\mathbf{x}_{p}\right)_{i+1 / 2, j} \Delta t D_{p}}{\left(1+D_{p} \Delta t\right) \rho_{p} \theta_{p_{i+1 / 2, j}}^{n}}\left[\nabla S\left(\mathbf{x}_{p}\right)_{i+1, j+1} \cdot \mathbf{e}_{x}\right] C_{1 p_{\tau}} \\
& b_{u_{t l}}=-\sum_{p} \frac{m_{p} N_{p} T\left(\mathbf{x}_{p}\right)_{i+1 / 2, j} \Delta t D_{p}}{\left(1+D_{p} \Delta t\right) \rho_{p} \theta_{p_{i+1 / 2, j}^{n}}^{n}}\left[\nabla S\left(\mathbf{x}_{p}\right)_{i, j+1} \cdot \mathbf{e}_{x}\right] C_{1 p_{\tau}} \\
& b_{u_{b r}}=-\sum_{p} \frac{m_{p} N_{p} T\left(\mathbf{x}_{p}\right)_{i+1 / 2, j} \Delta t D_{p}}{\left(1+D_{p} \Delta t\right) \rho_{p} \theta_{p_{i+1 / 2, j}}^{n}}\left[\nabla S\left(\mathbf{x}_{p}\right)_{i+1, j-1} \cdot \mathbf{e}_{x}\right] C_{1 p_{\tau}} \\
& b_{u_{b l}}=-\sum_{p} \frac{m_{p} N_{p} T\left(\mathbf{x}_{p}\right)_{i+1 / 2, j} \Delta t D_{p}}{\left(1+D_{p} \Delta t\right) \rho_{p} \theta_{p_{i+1 / 2, j}}^{n}}\left[\nabla S\left(\mathbf{x}_{p}\right)_{i, j-1} \cdot \mathbf{e}_{x}\right] C_{1 p_{\tau}} .
\end{aligned}
$$

The constant includes the gravitational term and transient old time term

$$
\begin{gathered}
C_{u}=\frac{\rho_{g} \delta x \Delta y}{\Delta t}\left(\theta_{g} u_{g}\right)_{i+1 / 2, j}^{n}+\delta x \Delta y \theta_{g}^{n} \rho_{g} g \\
-\sum_{p} \frac{m_{p} N_{p} T\left(\mathbf{x}_{p}\right)_{i+1 / 2, j} \Delta t D_{p}}{\left(1+D_{p} \Delta t\right) \rho_{p} \theta_{p_{i+1 / 2, j}^{n}}^{n}}\left[\nabla S\left(\mathbf{x}_{p}\right)_{i, j} \cdot \mathbf{e}_{x}\right] C_{2 p_{\tau}} \\
-\sum_{p} \frac{m_{p} N_{p} T\left(\mathbf{x}_{p}\right)_{i+1 / 2, j} \Delta t D_{p}}{\left(1+D_{p} \Delta t\right) \rho_{p} \theta_{p_{i+1 / 2, j}}^{n}}\left[\nabla S\left(\mathbf{x}_{p}\right)_{i+1, j} \cdot \mathbf{e}_{x}\right] C_{2 p_{\tau}}
\end{gathered}
$$




$$
\begin{gathered}
-\sum_{p} \frac{m_{p} N_{p} T\left(\mathbf{x}_{p}\right)_{i+1 / 2, j} \Delta t D_{p}}{\left(1+D_{p} \Delta t\right) \rho_{p} \theta_{p_{i+1 / 2, j}^{n}}^{n}}\left[\nabla S\left(\mathbf{x}_{p}\right)_{i+1, j+1} \cdot \mathbf{e}_{x}\right] C_{2 p_{\tau}} \\
-\sum_{p} \frac{m_{p} N_{p} T\left(\mathbf{x}_{p}\right)_{i+1 / 2, j} \Delta t D_{p}}{\left(1+D_{p} \Delta t\right) \rho_{p} \theta_{p_{i+1 / 2, j}}^{n}}\left[\nabla S\left(\mathbf{x}_{p}\right)_{i, j+1} \cdot \mathbf{e}_{x}\right] C_{2 p_{\tau}} \\
-\sum_{p} \frac{m_{p} N_{p} T\left(\mathbf{x}_{p}\right)_{i+1 / 2, j} \Delta t D_{p}}{\left(1+D_{p} \Delta t\right) \rho_{p} \theta_{p_{i+1 / 2, j}^{n}}^{n}\left[\nabla S\left(\mathbf{x}_{p}\right)_{i+1, j-1} \cdot \mathbf{e}_{x}\right] C_{2 p_{\tau}}} \\
-\sum_{p} \frac{m_{p} N_{p} T\left(\mathbf{x}_{p}\right)_{i+1 / 2, j} \Delta t D_{p}}{\left(1+D_{p} \Delta t\right) \rho_{p} \theta_{p_{i+1 / 2, j}}^{n}}\left[\nabla S\left(\mathbf{x}_{p}\right)_{i, j-1} \cdot \mathbf{e}_{x}\right] C_{2 p_{\tau}} .
\end{gathered}
$$

The constants $C_{1 p_{\tau}}$ and $C_{1 p_{\tau}}$ are given by equations $49 \mathrm{a}$ and $49 \mathrm{~b}$. 


\section{B. Pressure equation coefficients}

The pressure correction equation is formed by substituting velocities from the momentum equations into the volume continuity equation. The velocity is $\mathbf{u}=\mathbf{u}^{*}+\delta \mathbf{u}$ and the pressure is $p=p^{*}+\delta p$, where $\mathbf{u}^{*}$ is the calculated velocity field using the estimated pressure $p^{*}$. The true pressure, $p$, is the sum of estimated pressure, $p^{*}$, plus a correction pressure, $\delta p$. The correction velocity is $\delta \mathbf{u}$. Substituting velocities from the momentum equations into the gas continuity equation, using a fixed gas volume fraction, and neglecting neighbor correction-velocities, the pressure equation (50) is

$$
\begin{aligned}
& a_{p_{c c} c_{i, j}} \delta p_{i, j}+a_{p_{l l}} \delta p_{i-j}+a_{p_{r r}} \delta p_{i, j} p_{i+1, j}+a_{p_{b b}} \delta p_{i, j} p_{i, j-1}+a_{p_{t t} t, j} \delta p_{i, j+1} \\
& +a_{p b l} \delta p_{i, j} p_{i-1, j-1}+a_{p_{b r}} \delta p_{i+j} p_{i+1, j-1}+a_{p_{l l_{i, j}}} \delta p_{i-1, j+1}+a_{p_{t r}} \delta p_{i+1, j}=-e r r_{i, j} .
\end{aligned}
$$

The $\operatorname{err}_{i, j}$ results from using $\mathbf{u}^{*}$ in solving of the continuity equation. The pressure equation coefficients are

$$
\begin{aligned}
& a_{p_{c c} i, j}=A_{x_{j}} \tilde{\theta}_{g, x_{i, j}} a_{u l_{l, j}}-A_{x_{j}} \tilde{\theta}_{g, x_{i-1, j}} a_{u_{r r}}+A_{y_{i}} \tilde{\theta}_{g, y_{i, j}} a_{v_{b b}, j}-A_{y_{i}} \tilde{\theta}_{g, y_{i, j-1}} a_{u_{t t}} \\
& a_{p_{r r_{i}, j}}=A_{x_{j}} \tilde{\theta}_{g, x_{i, j}} a_{u_{r r}}+A_{y_{i}} \tilde{\theta}_{g, y_{i, j}} a_{v b r_{i, j}}-A_{y_{i}} \tilde{\theta}_{g, y_{i, j-1}} a_{v t r i, j-1} \\
& a_{p_{l l_{i, j}}}=-A_{x_{j}} \tilde{\theta}_{g, x_{i-1, j}} a_{u l l_{i-1, j}}+A_{y_{i}} \tilde{\theta}_{g, y_{i, j}} a_{v_{b l} l_{i, j}}-A_{y_{i}} \tilde{\theta}_{g, y_{i, j-1}} a_{v_{t} t_{i, j-1}} \\
& a_{p_{t t_{i, j}}}=A_{y_{i}} \tilde{\theta}_{g, y_{i, j}} a_{v_{t t_{i, j}}}+A_{x_{j}} \tilde{\theta}_{g, x_{i, j}} a_{u_{t l} l_{i, j}}-A_{x_{j}} \tilde{\theta}_{g, x_{i-1, j}} a_{u_{t r}}{ }_{i-1, j} \\
& a_{p_{b b_{i, j}}}=-A_{y_{i}} \tilde{\theta}_{g, y_{i, j-1}} a_{v b b_{i, j-1}}+A_{x_{j}} \tilde{\theta}_{g, x_{i, j}} a_{u b l}-A_{x_{j}} \tilde{\theta}_{g, x_{i-1, j}} a_{u b r_{i-1, j}} \\
& a_{p_{t r_{i, j}}}=A_{x_{j}} \tilde{\theta}_{g, x_{i, j}} a_{u_{t r}}+A_{y_{i}} \tilde{\theta}_{g, y_{i, j}} a_{v t r_{i, j}} \\
& a_{p_{b l} l_{i, j}}=-A_{x_{j}} \tilde{\theta}_{g, x_{i-1, j}} a_{u b l}+A_{y_{i}} \tilde{\theta}_{g, y_{i, j-1}} a_{v b l_{i, j-1}} \\
& a_{p_{t t_{i, j}}}=-A_{x_{j}} \tilde{\theta}_{g, x_{i-1, j}} a_{u_{t l} l_{i-1, j}}+A_{y_{i}} \tilde{\theta}_{g, y_{i, j}} a_{v_{t} l_{i, j}} \\
& a_{p_{b r} r_{i, j}}=A_{x_{j}} \tilde{\theta}_{g, x_{i, j}} a_{u_{b r}}+A_{y_{i}} \tilde{\theta}_{g, y_{i, j-1}} a_{v_{b r}}
\end{aligned}
$$

where $A_{x_{j}}$ is the area normal to the u-velocity and $A_{y_{i}}$ is the area normal to the v-velocity. Coefficients $a_{u}$ and $a_{v}$ are from the algebraic momentum equations given by equations (46) and (47). Volume fractions are intermediate values at cell faces, where $\tilde{\theta}_{g, x}$ corresponds to the $u-$ velocity, staggered grid, and $\tilde{\theta}_{g, y}$ corresponds to the v-velocity, staggered grid. 
The continuum continuity equation contains the rate of change of fluid volume fraction. The new-time volume fraction can be implicitly included through iterations, where the particle volume fraction is calculated while holding the other implicit variables fixed.

The particle volume fraction also can be directly included in the pressure equation through the continuity equation time dependent term. The true volume fraction is $\theta_{g}=\theta_{g} *+\delta \theta_{g}$, where $\theta_{g} *$ is the calculated volume fraction calculated using $p^{*}$ and $\mathbf{u}^{*}$, and $\delta \theta_{g}$ is the correction value. The particle volume fraction is calculated from equation (57) using $p^{*}$ and $\mathbf{u}^{*}$, and in symbolic form, is

$$
\theta_{p_{i, j}}^{* n+1}=\tilde{\theta}_{p_{i, j}}+\sum_{\text {Neighbors }} A \theta_{p}^{* n+1}+\sum_{\text {Neighbors }} B \mathbf{u}^{*_{n+1}} *+\sum_{\text {Neighbors }} C p^{*_{n+1}}+D_{\theta} .
$$

The true volume fraction is

$$
\theta_{p_{i, j}}^{n+1}=\tilde{\theta}_{p_{i, j}}+\sum_{\text {Neighbors }} A \theta_{p}^{n+1}+\sum_{\text {Neighbors }} B \mathbf{u}^{n+1}+\sum_{\text {Neighbors }} C p^{n+1}+D_{\theta} .
$$

Subtracting the true and calculated volume fractions and neglecting neighbor correction volume fraction terms gives the correction volume fraction which depends on $\delta \mathbf{u}$ and $\delta \mathrm{p}$,

$$
\delta \theta_{p_{i, j}}=\sum_{\text {Neighbors }} B \delta \mathbf{u}+\sum_{\text {Neighbors }} C \delta p
$$

The rate of change of the continuum volume fraction is

$$
\frac{\partial \theta_{g}}{\partial t}=-\frac{\partial \theta_{p}}{\partial t}
$$

The continuum continuity equation time dependent term is

$$
\begin{gathered}
\frac{\left(V \theta_{g}\right)_{i, j}^{n+1}-\left(V \theta_{g}\right)_{i, j}^{n}}{\Delta t}=\frac{\left(V \theta_{g}^{*}\right)_{i, j}^{n+1}+\left(V \delta \theta_{g}\right)_{i, j}-\left(V \theta_{g}\right)_{i, j}^{n}}{\Delta t} \\
\frac{\left(V \theta_{g}^{*}\right)_{i, j}^{n+1}-\left(V \theta_{g}\right)_{i, j}^{n}}{\Delta t}
\end{gathered}
$$

where

is the same term used when an iteration loop is employed to implicitly couple variables. The remainder, $\frac{\left(V \delta \theta_{g}\right)_{i, j}}{\Delta t}$, is expressed in terms of $\delta \mathbf{u}$ and $\delta p$, and these terms are added to the $\delta \mathbf{u}$ and $\delta p$ terms from convection. The algebraic pressure equation is the same as given above with coefficients 


$$
\begin{aligned}
& a_{p_{c c}, j}=A_{x r} a_{u l l_{i, j}}-A_{x l} a_{u_{r r}+1, j}+A_{y t} a_{v_{b b_{i, j}}}-A_{y b} a_{v_{t t} i, j-1}-K_{i, j} a_{\theta_{c c_{i, j}}} \\
& a_{p_{r i, j}}=A_{x r} a_{u_{r r}, j}+A_{y t} a_{v b r_{i, j}}-A_{y b} a_{v_{t r}, j-1}-K_{i, j} a_{\theta_{r i, j}} \\
& a_{p_{l l_{i, j}}}=-A_{x l} a_{u_{l l} l_{i-1, j}}+A_{y t} a_{v_{b l} l_{i, j}}-A_{y b} a_{v_{t l}, j-1}-K_{i, j} a_{\theta_{l i, j}} \\
& a_{p_{t t_{i, j}}}=A_{y t} a_{v_{t t}}+A_{x r} a_{u_{t l} l, j}-A_{x l} a_{u_{t r} r_{i-1, j}}-K_{i, j} a_{\theta_{t t_{i, j}}} \\
& a_{p_{b b_{i, j}}}=-A_{y b} a_{v_{b b}, j-1}+A_{x r} a_{u_{b l}, j}-A_{x l} a_{u_{b r_{i-1, j}}}-K_{i, j} a_{\theta_{b b_{i, j}}} \\
& a_{p_{t r} i, j}=A_{x r} a_{u_{t r}}+A_{i, j} a_{v_{t r}}-K_{i, j} a_{\theta_{t i, j}} \\
& a_{p b l_{i, j}}=-A_{x l} a_{u b l_{i-1, j}}+A_{y b} a_{v_{b l}, j-1}-K_{i, j} a_{\theta_{b l, j}} \\
& a_{p_{t l}, j}=-A_{x l} a_{u_{t l} l-1, j}+A_{y t} a_{v_{t l}}-K_{i, j} a_{\theta_{t l_{i, j}}} \\
& a_{p_{b r} r_{i, j}}=A_{x r} a_{u b r_{i, j}}+A_{y b} a_{v b r_{i, j-1}}-K_{i, j} a_{\theta_{b r, j}}
\end{aligned}
$$

where

$$
\begin{gathered}
K_{i, j}=\frac{-V_{i, j}}{b_{\theta_{c c}} \Delta t} \\
A_{x l}=\tilde{\theta}_{g, x_{i-1, j}} A_{x j}+K_{i, j} g_{\theta_{l l i, j}} \\
A_{x r}=\tilde{\theta}_{g, x_{i, j}} A_{x j}-K_{i, j} g_{\theta_{r r_{i, j}}} \\
A_{y b}=\tilde{\theta}_{g, y_{i, j-1}} A_{y i}+K_{i, j} h_{\theta_{b b_{i, j}}} \\
A_{y t}=\tilde{\theta}_{g, y_{i, j}} A_{y i}-K_{i, j} h_{\theta_{t t i, j}} .
\end{gathered}
$$

The $a_{\theta}, h_{\theta}$ and $g_{\theta}$ coefficients are from the particle volume fraction equation (57). 


\section{Particle volume fraction equation coefficients}

The particle volume fraction equation is aproximated by linear algebraic equations with independent variables $u$-velocity, $v$-velocity and pressure. The particle volume fraction at cellcenter node $(i+1 / 2, j)$ is given by equation $(57)$ :

$$
\begin{gathered}
b_{\theta_{c c}} \theta_{i, j}^{n+1}=a_{\theta_{c c}} p_{i, j}^{n+1}+a_{\theta_{l l}} p_{i-1, j}^{n+1}+a_{\theta_{r r}} p_{i+1, j}^{n+1}+a_{\theta_{b b}} p_{i, j-1}^{n+1} a_{\theta_{t t}} p_{i, j+1}^{n+1}+a_{\theta_{b l}} p_{i-1, j-1}^{n+1} \\
+a_{\theta_{b r}} p_{i+1, j-1}^{n+1}+a_{\theta_{t l}} p_{i-1, j+1}^{n+1}+a_{\theta_{t r}} p_{i+1, j+1}^{n+1} \\
+b_{\theta_{l l}} \theta_{i-1, j}^{n+1}+b_{\theta_{r r}} \theta_{i+1, j}^{n+1}+b_{\theta_{b b}} \theta_{i, j-1}^{n+1} b_{\theta_{t t}} \theta_{i, j+1}^{n+1}+b_{\theta_{b l}} \theta_{i-1, j-1}^{n+1} \\
+b_{\theta_{b r}} \theta_{i+1, j-1}^{n+1}+b_{\theta_{t l}} \theta_{i-1, j+1}^{n+1}+b_{\theta_{t r}} \theta_{i+1, j+1}^{n+1} \\
+g_{\theta_{l l}} u_{g, i-1 / 2, j}^{n+1}+g_{\theta_{r}} u_{g, i+1 / 2, j}^{n+1}+g_{\theta_{b l}} u_{g, i-1 / 2, j-1}^{n+1}+g_{\theta_{b r}} u_{g, i+1 / 2, j-1}^{n+1} \\
+g_{\theta_{t l}} u_{g, i-1 / 2, j+1}^{n+1}+g_{\theta_{t r}} u_{g, i+1 / 2, j+1}^{n+1} \\
+h_{\theta_{b b}} v_{g, i, j-1 / 2}^{n+1}+h_{\theta_{t t}} v_{g, i, j+1 / 2}^{n+1}+h_{\theta_{b l}} v_{g, i-1, j-1 / 2}^{n+1}+h_{\theta_{b r}} v_{g, i+1, j-1 / 2}^{n+1} \\
+h_{\theta_{t l}} v_{g, i-1, j+1 / 2}^{n+1}+h_{\theta_{t r}} v_{g, i+1, j+1 / 2}^{n+1}+c_{\theta}=0 .
\end{gathered}
$$

The nodilization is shown below.

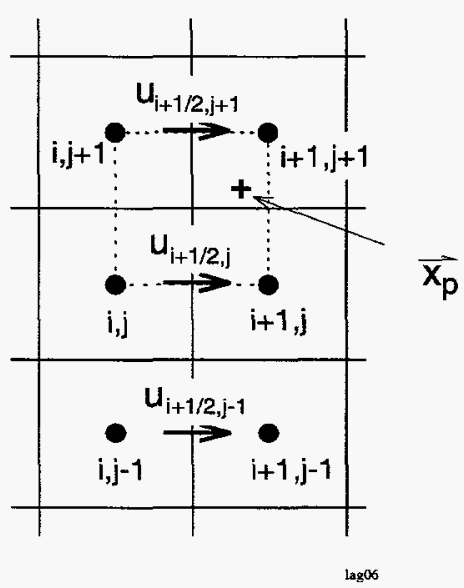

The coefficients on the $(i, j)$ gas u-velocity are

$$
g_{\theta_{r r}}=-\frac{\Delta t^{2}}{V_{i, j}} \sum_{p} \frac{m_{p} N_{p} T\left(\mathbf{x}_{p}\right)_{i+1 / 2, j} D_{p}}{\rho_{p}\left(1+D_{p} \Delta t\right)} \nabla S_{x}\left(\mathbf{x}_{p}\right)_{i, j}
$$




$$
\begin{aligned}
g_{\theta_{t r}} & =-\frac{\Delta t^{2}}{V_{i, j}} \sum_{p} \frac{m_{p} N_{p} T\left(\mathbf{x}_{p}\right)_{i+1 / 2, j+1} D_{p}}{\rho_{p}\left(1+D_{p} \Delta t\right)} \nabla S_{x}\left(\mathbf{x}_{p}\right)_{i, j} \\
g_{\theta_{l l}} & =-\frac{\Delta t^{2}}{V_{i, j}} \sum_{p} \frac{m_{p} N_{p} D_{p} T\left(\mathbf{x}_{p}\right)_{i-1 / 2, j}}{\rho_{p}\left(1+D_{p} \Delta t\right)} \nabla S_{x}\left(\mathbf{x}_{p}\right)_{i, j} \\
g_{\theta_{t l}} & =-\frac{\Delta t^{2}}{V_{i, j}} \sum_{p} \frac{m_{p} N_{p} T\left(\mathbf{x}_{p}\right)_{i-1 / 2, j+1} D_{p}}{\rho_{p}\left(1+D_{p} \Delta t\right)} \nabla S_{x}\left(\mathbf{x}_{p}\right)_{i, j} \\
g_{\theta_{b l}} & =\frac{\Delta t^{2}}{V_{i, j}} \sum_{p} \frac{m_{p} N_{p} T\left(\mathbf{x}_{p}\right)_{i-1 / 2, j-1} D_{p}}{\rho_{p}\left(1+D_{p} \Delta t\right)} \nabla S_{x}\left(\mathbf{x}_{p}\right)_{i, j} \\
g_{\theta_{b r}} & =\frac{\Delta t^{2}}{V_{i, j}} \sum_{p} \frac{m_{p} N_{p} T\left(\mathbf{x}_{p}\right)_{i+1 / 2, j-1} D_{p}}{\rho_{p}\left(1+D_{p} \Delta t\right)} \nabla S_{x}\left(\mathbf{x}_{p}\right)_{i, j} .
\end{aligned}
$$

The $\nabla S_{x}$ represents the $\mathrm{x}$-component of the gradient. The coefficients on the $(i, j)$ gas v-velocity are

$$
\begin{aligned}
& h_{\theta_{t t}}=-\frac{\Delta t^{2}}{V_{i, j}} \sum_{p} \frac{m_{p} N_{p} D_{p} T\left(\mathbf{x}_{p}\right)_{i, j+1 / 2}}{\rho_{p}\left(1+D_{p} \Delta t\right)} \nabla S_{y}\left(\mathbf{x}_{p}\right)_{i, j} \\
& h_{\theta_{t r}}=-\frac{\Delta t^{2}}{V_{i, j}} \sum_{p} \frac{m_{p} N_{p} D_{p} T\left(\mathbf{x}_{p}\right)_{i+1, j+1 / 2}}{\rho_{p}\left(1+D_{p} \Delta t\right)} \nabla S_{y}\left(\mathbf{x}_{p}\right)_{i, j} \\
& h_{\theta_{t l}}=-\frac{\Delta t^{2}}{V_{i, j}} \sum_{p} \frac{m_{p} N_{p} D_{p} T\left(\mathbf{x}_{p}\right)_{i-1, j+1 / 2}}{\rho_{p}\left(1+D_{p} \Delta t\right)} \nabla S_{y}\left(\mathbf{x}_{p}\right)_{i, j} \\
& h_{\theta_{b l}}=-\frac{\Delta t^{2}}{V_{i, j}} \sum_{p} \frac{m_{p} N_{p} D_{p} T\left(\mathbf{x}_{p}\right)_{i-1, j-1 / 2}}{\rho_{p}\left(1+D_{p} \Delta t\right)} \nabla S_{y}\left(\mathbf{x}_{p}\right)_{i, j} \\
& h_{\theta_{b b}}=-\frac{\Delta t^{2}}{V_{i, j}} \sum_{p} \frac{m_{p} N_{p} D_{p} T\left(\mathbf{x}_{p}\right)_{i, j-1 / 2}}{\rho_{p}\left(1+D_{p} \Delta t\right)} \nabla S_{y}\left(\mathbf{x}_{p}\right)_{i, j}
\end{aligned}
$$




$$
h_{\theta_{b r}}=-\frac{\Delta t^{2}}{V_{i, j}} \sum_{p} \frac{m_{p} N_{p} D_{p} T\left(\mathbf{x}_{p}\right)_{i+1, j-1 / 2}}{\rho_{p}\left(1+D_{p} \Delta t\right)} \nabla S_{y}\left(\mathbf{x}_{p}\right)_{i, j} .
$$

The $\nabla S_{y}$ represents the y-component of the gradient:

$$
\begin{aligned}
& a_{\theta_{c c}}=-\frac{\Delta t^{2}}{V_{i, j}} \sum_{p} \frac{m_{p} N_{p}}{\rho_{p}{ }^{2}\left(1+D_{p} \Delta t\right)} \nabla S\left(\mathbf{x}_{p}\right)_{i, j} \cdot \nabla S\left(\mathbf{x}_{p}\right)_{i, j} \\
& a_{\theta_{r r}}=-\frac{\Delta t^{2}}{V_{i, j}} \sum_{p} \frac{m_{p} N_{p}}{\rho_{p}{ }^{2}\left(1+D_{p} \Delta t\right)} \nabla S\left(\mathbf{x}_{p}\right)_{i, j} \cdot \nabla S\left(\mathbf{x}_{p}\right)_{i+1, j} \\
& a_{\theta_{t r}}=-\frac{\Delta t^{2}}{V_{i, j}} \sum_{p} \frac{m_{p} N_{p}}{\rho_{p}{ }^{2}\left(1+D_{p} \Delta t\right)} \nabla S\left(\mathbf{x}_{p}\right)_{i, j} \cdot \nabla S\left(\mathbf{x}_{p}\right)_{i+1, j+1} \\
& a_{\theta_{t t}}=-\frac{\Delta t^{2}}{V_{i, j}} \sum_{p} \frac{m_{p} N_{p}}{\rho_{p}{ }^{2}\left(1+D_{p} \Delta t\right)} \nabla S\left(\mathbf{x}_{p}\right)_{i, j} \cdot \nabla S\left(\mathbf{x}_{p}\right)_{i, j+1} \\
& a_{\theta_{l l}}=-\frac{\Delta t^{2}}{V_{i, j}} \sum_{p} \frac{m_{p} N_{p}}{\rho_{p}{ }^{2}\left(1+D_{p} \Delta t\right)} \nabla S\left(\mathbf{x}_{p}\right)_{i, j} \cdot \nabla S\left(\mathbf{x}_{p}\right)_{i-1, j} \\
& a_{\theta_{t t}}=-\frac{\Delta t^{2}}{V_{i, j}} \sum_{p} \frac{m_{p} N_{p}}{\rho_{p}{ }^{2}\left(1+D_{p} \Delta t\right)} \nabla S\left(\mathbf{x}_{p}\right)_{i, j} \cdot \nabla S\left(\mathbf{x}_{p}\right)_{i, j-1} \\
& a_{\theta_{t l}}=-\frac{\Delta t^{2}}{V_{i, j}} \sum_{p} \frac{m_{p} N_{p}}{\rho_{p}{ }^{2}\left(1+D_{p} \Delta t\right)} \nabla S\left(\mathbf{x}_{p}\right)_{i, j} \cdot \nabla S\left(\mathbf{x}_{p}\right)_{i-1, j+1} \\
& a_{\theta_{b l}}=-\frac{\Delta t^{2}}{V_{i, j}} \sum_{p} \frac{m_{p} N_{p}}{\rho_{p}{ }^{2}\left(1+D_{p} \Delta t\right)} \nabla S\left(\mathbf{x}_{p}\right)_{i, j} \cdot \nabla S\left(\mathbf{x}_{p}\right)_{i-1, j-1} \\
& a_{\theta_{b r}}=-\frac{\Delta t^{2}}{V_{i, j}} \sum_{p} \frac{m_{p} N_{p}}{\rho_{p}{ }^{2}\left(1+D_{p} \Delta t\right)} \nabla S\left(\mathbf{x}_{p}\right)_{i, j} \cdot \nabla S\left(\mathbf{x}_{p}\right)_{i+1, j-1} .
\end{aligned}
$$

